\title{
The Type Discipline of Behavioral Separation
}

\author{
Luís Caires João C. Seco \\ CITI and Departamento de Informática \\ Faculdade de Ciências e Tecnologia, Universidade Nova de Lisboa
}

(long version with Appendix - TR DI FCT UNL draft of September, 2012)

\begin{abstract}
We introduce the concept of behavioral separation as a general principle for disciplining interference in higher-order imperative concurrent programs, and present a type-based approach that systematically develops the concept in the context of an ML-like language extended with concurrency and synchronization primitives. Behavioral separation builds on notions originally introduced for behavioral type systems and separation logics, but shifts the focus from the separation of static program state properties towards the separation of dynamic usage behaviors of runtime values. Behavioral separation types specify how values may be safely used by client code, and can enforce fine-grained interference control disciplines while preserving compositionality, information hiding, and flexibility. We illustrate how our type system, even if based on a small set of general primitives, is already able to tackle fairly challenging program idioms, involving aliasing at various types, concurrency with first-class threads, manipulation of linked data structures, behavioral borrowing, and invariant-based separation.
\end{abstract}

\section{Introduction}

The purpose of this work is to introduce and develop the concept of behavioral separation as a general principle for disciplining interference in higher-order imperative concurrent programs.

Statically verifying that higher-order imperative programs do not go wrong in the presence of possible interference has proven to be a challenging task, and a fertile ground for research since the seminal work of Reynolds [32]. In general, two program fragments interfere when the effects generated by one fragment may change the state visible to the other, typically due to aliasing or to concurrency. Some forms of interference are "bad", and may cause catastrophic failure, such as read/write races when accessing the same memory cell. Other forms of interference are "good" and even required, such as the interference between producer and consumer running concurrently, and sharing a thread-safe stateful queue, or the interference between the head and tail references of a linked list data structure. "Interference is the essence of concurrency", as Cliff Jones has frequently written [24].

An ongoing challenge to overcome is then to find techniques for disciplining interference between different usages of the same objects, so to ensure safety while being able to address increasingly sophisticated programming idioms.
Significant advances have been achieved recently towards approaching these general goals, in particular by the separation logics of O'Hearn and Reynolds [33, 30] and by substructural type and effect systems, e.g., $[1,8,3]$. In particular, separation logic supports expressive forms of local reasoning, based on the use of the separating conjunction in combination with fractional permissions $[9,5]$ to characterize the fine structure of program states. Extending such state-based techniques to tackle the sophisticated program idioms arising in modern higher-order imperative concurrent programming is thus both promising and challenging [36].

In this work, we radically depart from a state-based view towards a behavioral view of program assertions, by introducing a notion of behavioral separation type structure. Behavioral separation builds on notions originally introduced for behavioral type systems and separation logics, but shifts the focus from the separation of static program state properties towards the separation of dynamic usage behaviors of runtime values. We thus introduce a type-based approach that systematically develops the concept of behavioral separation to enforce safety of programs, ruling out "bad" interferences in the presence of aliasing and concurrency. Our presentation is grounded on a core ML-like language, with higher-order functions, heap allocated variables, tuples, variants, and concurrency, which we pick as a convenient abstraction for other languages supporting higher-order imperative concurrent programming.

Behavioral types $[19,14,22]$ based on process algebras, have been introduced with the aim of characterizing the interface of a process not just as a specification of the static type of exchanged messages, but also as specification of its dynamic behavior. Likewise, our behavioral separation types specify how program values may be safely used by client code, but are able to enforce finegrained interference control disciplines. A key novelty of our approach consists in uniformly combining in the same type structure "temporal" operations, such as sequential separation, important to capture trace constraints, with "spatial" operations, such as parallel separation and isolation, important to capture aliasing and concurrency. Remarkably, we carry out our development in the context of a clean substructural type theory, in which all type operators satisfy natural algebraic properties, and is based on a $\lambda$-calculus extended with imperative references and concurrency constructs.

Behavioral separation types also promote information hiding, compositionality, and flexibility, since type assertions talk about separation constraints on usage behaviors as externally perceived by the programs which use them, rather than about the internal structure of program state or program code. As will be clear from our examples, behavioral separation types are also expressive and flexible enough for proving safety of programs combining features still challenging for (and even out of reach of) existing proof methods, including general higher-order store, aliasing / sharing at all types, linked data structures, borrowing of local behavior, first-class threads, and even invariant-based separation, based on typed synchronization constructs. 


\section{Overview}

In this section, we motivate the general concept of behavioral separation, informally introducing on the way our core programming language and explaining the various behavioral separation type operators. We proceed by going through a sequence of examples. Consider the following implementation of a collection abstract data type, where we assume the list elements to be natural numbers, and the representation data structure to be a linked list.

$$
\begin{aligned}
& \text { let } n e w N o d e=\lambda[] \text {.var next, elt in } \\
& {[\text { setElt }=\lambda e .(\text { elt }:=e) \text {, }} \\
& \text { getElt }=\text { elt } \\
& \text { setNext }=\lambda p .(\text { next }:=p) \text {, } \\
& \text { getNext }=\text { next ] in }
\end{aligned}
$$

We define four operations on collections: the initializer init, which sets the collection identifier (a string); the getId operation, which returns the collection identifier; the add operation, which adds a new element to the collection; and the scan operation, which traverses the linked list, visiting each node in sequence. We model ADT "objects" by tuples of closures sharing memory locations, and "classes" by object generating functions, along standard lines. In our language, tuple fields are bound to expressions (quoted code), to be evaluated only after field selection (as e.g. in [35]). The var $x$ in $e$ block creates a ML-style heap allocated variable, where the created cell can survive the lifetime of the body $e$, embedded in the value returned (cf. let $x=\operatorname{ref}(\mathbf{n i l})$ in $e$ in ML).

In the code shown above, the private $h d$ variable refers to the head of the linked list, and is shared by the add and scan operations. We represent references to list elements by variant values, with options NULL(nil) abbreviated NULL and representing the null reference, and $\operatorname{NODE}(n)$, representing a list node. $\operatorname{In} \operatorname{NODE}(n), n$ is a tuple with fields setElt, getElt, setNext, and getNext. Notice that the latter two fields access the heap variable next, which references the next node, if any, and is local to the given node.

Using standard functional and product types we would assign to newColl a type as $0 \rightarrow S C$, where 0 is a "unit" type, and $S C$ a record type representing the collection ADT "objects". Such a record type would essentially specify a flat interface, listing the operations available, each one modeled as a typed record field. In our system, types specify (fine grained) value usage behaviors, rather than value structure. As a first example consider the type

$$
S C \triangleq i n i t: \text { str } \mapsto 0 ;(\text { getId: } \text { str \& add:nat } \mapsto 0 \text { \& scan:0)* }
$$

The type $S C$ specifies a possible usage behavior offered by a collection. Intuitively, the type $S C$ says that collections may be used by first calling the init "method", and then the getId, add, and scan "methods", in iterated choice. First, it offers a label selection usage, denoted by the label selection type init:str $\mapsto 0$. The usage consists in selecting the init label to get a value of type str $\mapsto 0$. The stop type 0 specifies that no usage is available.

The operator $(-) \mapsto(-)$ is our primitive functional type. $U \mapsto V$ is a type for functions which do not interfere unsafely with their argument, and specifies a single usage of a value as a function, at the appropriate argument $U$ and return type $V$. The $U \mapsto V$ does not correspond to a standard function type $U \rightarrow V$, which can nevertheless be encoded in our system as will become clear later. It does neither correspond to the linear arrow, nor to the arrows of separation $[33,30]$ or bunched logic [29], even if it is closely connected to all of these. In a stateful, concurrent programming world, there are too many ways of using a function object. In conjugation with other type constructors, the functional type $U \mapsto V$ allows much of such variety to be modularly approached.

Sequencing of behaviors is expressed by the sequential separation type constructor $(-) ;(-)$. A value typed by $U ; V$ first offers to clients the usage behavior $U$ and only after $V$. In our example, after the init:str $\mapsto 0$ usage behavior, the collection value offers a usage (getId:str \& add:nat $\rightarrow 0$ \& scan:0)* This last type specifies the iterated choice between the selection of fields getId, $a d d$, and scan, each one yielding a value of respectively type str, nat $\mapsto 0$, and 0 . Choice between alternative behaviors is expressed using intersection types. A value typed by $U \& V$ offers to clients the choice between behaviors $U$ and $V$, since it can provide both $U$ and $V$, alternatively. The star type $U^{*}$ denotes the iteration of $U$, defined by $U^{*} \triangleq \operatorname{rec}(X)(0 \&(U ; X))$.

Clearly, the type operators just described may express rich sequential protocols for program values. Still, they are not expressive enough to address aliased or concurrent usages, due to the strict linearity they enforce. A more flexible specification would allow, after initialization, the getId operation to be always available for execution, concurrently with a $a d d$ or a scan operation. On the other hand, a concurrent execution of the $a d d$ and scan operations may lead to unsafe interference, due to a read/write race on $h d$. To express this behavioral usage we use the parallel separation type constructor $(-) \mid(-)$.

In general, a $U \mid V$ type asserts that behaviors $U$ and $V$ may be safely used by causally independent clients, either due to aliasing or concurrency, without incurring in unsafe interference. Such a parallel usage only completes when both behaviors $U$ and $V$ complete. Exploring the parallel type, we may assign to function new Coll the more flexible type $0 \mapsto C C$ where

$$
C C \triangleq(\text { init: } \text { str } \mapsto 0) ;\left(\text { getId:str } \mid(! \text { scan }: 0 ; \text { add:nat } \mapsto 0)^{*}\right)
$$

The type $C C$ asserts that, after initialization, a collection provides two independently usable behaviors, one of type !getId:str and other of type $(! \operatorname{scan}: 0 \text {; add:nat } \mapsto 0)^{*}$, composed using the parallel separation type constructor $(-) \mid(-)$.

The type constructor !(-), used in !getId:str and !scan:0, specifies an unbounded number, possibly zero, of separated parallel usages (parallel in the same sense of $(-) \mid(-)$ ). In particular, the type !getId:str allows an unbounded number of (possibly concurrent) aliases to access the field getId:str. Then, the type $(! \text { scan: } 0 \text {; add:nat } \rightarrow 0)^{*}$ specifies a usage consisting of the interleaved repetition of some parallel usages of the scan:0 behavior followed by the $a d d$ :nat $\rightarrow 0$ behavior. Only after all the scan operations selected in the !scan:0 phase conclude, will add:nat $\mapsto 0$ become again available. Since there is no obligation to select some scan:0 operation in the !scan:0 phase, the type $C C$ also allows any number of $a d d$ :nat $\mapsto 0$ operations to be sequentially performed.

Notice that the behavior of a newly created collection $c$ is completely separated, or isolated, from context: no behavioral dependencies exist between $c: C C$ and the behavior of other values in a running program using the collection. We denote isolation by a type operator $\circ(-)$, which also plays an important role in our framework. We thus assign to the function newColl the type $0 \mapsto \circ C C$.

Let us now consider some code snippets using the collection type just defined, and discuss valid (and invalid) typings.

let $c=$ newColl [] in (c.init "my"); c.scan; (c.add 1)
let $c=$ newColl [] in (c.init "my"); (c.add 1$) ;$ c.getId; c.scan 
Both code fragments are validated by our type system. In the first one, it is clear that the usage of $c$ follows the intended type. In the second one, the intended usage type of $c$ is also not violated, even if behaviors that appear parallel separated in the type (e.g., c:getId:str and $c: s c a n: 0)$ are sequentially used in the code. Clearly, if a value may be safely used according to $U \mid V$, it may also be safely used according to $U ; V$. Subsumption principles as this one are captured by subtyping, a pre-order on types written $U<: V$. In particular, subtyping satisfies the exchange law [17]

$$
(A ; C) \mid(B ; D)<:(A \mid B) ;(C \mid D)
$$

of which $U \mid V<: U ; V$ is a special case.

The next examples illustrates behavioral "borrowing", where fragments of the behavior of $c$ are temporarily used by some function, before being given back to the caller context.

$$
\begin{aligned}
& \text { let } c=\text { newColl }[] \text { in } \\
& \text { let } f=\lambda x .(x . \text { init "your" }) \text { in }(f c) ;(\text { c.add } 2) \\
& \text { let } c=n e w C o l l[] \text { in } \\
& \text { let } g=\lambda x .(x . s c a n) \text { in } \\
& \quad(\text { c.init "my" }) ;(g c) ; c . s c a n ;(c . a d d 2) ;(g c)
\end{aligned}
$$

In the second case the borrowing function is used twice, at different places of the global behavior. The borrowed type is declared in the function domain, e.g., $f$ : (init:str $\mapsto 0) \mapsto 0$. On the other hand

$$
\begin{aligned}
& \text { let } c=\text { newColl }[] \text { in } \\
& \quad \text { let } h=\lambda x .(x . \text { init "your") in (c.add } 2) ;(h c)
\end{aligned}
$$

attempts to use $a d d$ before init, and is rejected by our type system.

More interesting examples illustrate borrowing of behavior through the store, which our type system is able to handle in a natural way, even in a higher-order setting. Consider the following code snippet. It respects the expected behavioral constraints on the value $c$ and the heap allocated variable $a$, even if the behavior of $c$ is temporarily accessible at $a$, and is in fact typable in our system.

$$
\begin{aligned}
& \text { let } c=\text { newColl }[] \text { in var } a \text { in } \\
& \quad(a:=c ;(\text { a.init "my" }) ;(\text { a.add } 1) ;(\text { a.add } 1) ; \text { c.scan }))
\end{aligned}
$$

Heap variables are assigned behavioral separation types expressed in terms of use use, read $\operatorname{rd}(U)$ and write $\mathrm{wr}(U)$ capabilities, and related by subtyping axioms. We show some of them here

$$
\begin{aligned}
& \text { var <: use; var use <: } \operatorname{wr}(U) ; \operatorname{rd}(U) \quad \operatorname{rd}(! U)<: \operatorname{lrd}(U) \\
& \operatorname{rd}(U ; V)<: \operatorname{rd}(U) ; \operatorname{rd}(V) \quad \operatorname{rd}(U \mid V)<: \operatorname{rd}(U) \mid \operatorname{rd}(V)
\end{aligned}
$$

The first two axioms say that a single use of a variable consists in writing on it a value of type $U$, followed by a matching read phase. The following axioms specify how the reading phase may be behaviorally separated, depending on the type of the stored value.

The next example illustrates higher-order borrowing through the store, the function attached to the $a d d$ field is itself stored in memory, before being called.

$$
\begin{aligned}
& \text { let } c=\text { newColl }[] \text { in }((\text { c.init "my"); } \\
& \quad \operatorname{var} a \text { in }(a:=\text { c. } a d d ;(a 1) ; c . s c a n))
\end{aligned}
$$

This code does not violate any constraints imposed by the type of $c$, even if a collection "method" (a functional value) is extracted by selecting c. $a d d$ and stored in the temporary heap variable $a$.

These last two examples get past our typing discipline, because the type system keeps track of global separation constraints between all the values in the scope, and relies on sequential and parallel frame reasoning to locally replace behaviors in behavioral separation type assertions. In the last example, the function of type nat $\mapsto 0$ is required to be used (exactly once) before $s c a n$ is selected in $c$, even if its behavior is temporarily stored in the variable $a$, respecting the initial footprint of $c$. add of in the global behavioral separation type. The type pre-condition of $a:=c . a d d$ is $a$ :use $\mid(c$ :add:nat $\mapsto 0 ; c: s c a n: 0 ; \cdots)$, and the post-condition is $a: \operatorname{rd}(a d d:$ nat $\mapsto 0) ; c: s c a n: 0 ; \cdots$. Notice that this last type sequentially constrains the behaviors of $a$ and $c$, forcing $a$ to be read before using $c$. The ability to specify global separation constraints, involving several values, seems essential for the expressiveness of our system. As a further illustration of this point, consider

$$
\text { let } c=\text { new Coll [] in let } m=\text { c.init in c.scan }
$$

Here, the init qualifier is selected, but since the associated functional behavior (bound to $m$ ) is not actually exercised, the initialization of the local $h d$ variable is not performed, causing the scan operation to "crash" in the case expression. Of course, this code does not typecheck under our current assumptions, since it does not preserve the frame conditions imposed by the intended behavioral separation protocols. Indeed, c.scan would need to be typed under the pre-condition $(m: \operatorname{str} \mapsto 0) ; c$ :scan:0; $\cdots$, which states that $m$ must be used (as a function) before progressing with the continuation behavior of collection $c$, which is not possible.

The type system systematically uses local reasoning and frame principles on behavioral separation assertions to compute the effects of program fragments in a modular way. As a further example, consider a case of function application, as in

$$
\begin{aligned}
& \operatorname{var} s \text { in }(s:=\text { "hi"; } \\
& \quad \text { let } F=\lambda x .(\text { let } c=\text { newColl }[] \text { in }(\text { c.init } x ; c)) \text { in } \\
& \quad(\text { let } u=(F s) \text { in }(u . a d d 1)))
\end{aligned}
$$

Function $F$ returns an initialized collection. Before typing $(F s)$ the type assertion is $s: \operatorname{rd}(\operatorname{str})$; $\operatorname{var} \mid F:(\operatorname{str} \mapsto \circ C C)$. We assume that the type of strings str is shareable ( str <: !str), so that reading from $s$ does not "empty" the variable. To type function application, we collect the footprints of argument and function as $(s: \operatorname{rd}(\operatorname{str}) \mid F:(\operatorname{str} \mapsto \circ C C)) ; s: r d($ str $)$; var. After typing let $u=\left(\begin{array}{ll}F & s\end{array}\right)$, the type assertion is $u: \circ C C ; s: \operatorname{rd}(\mathrm{str})$; var. Apparently, this says that $u$ must be fully used as $\circ C C$ before the variable $s$ can be read again, which is not sensible. However, since the behavior $\circ C C$ is isolated, as expressed by $\circ(-)$, any use of $s$ cannot causally depend upon it: by the subtyping principle $(\circ U) ; V<:(\circ U) \mid V$, we actually reach $u: \circ C C \mid s: r d($ str $)$; var. Isolated types offer a safe escape from the strict locality discipline, allowing isolated behaviors to be fully (and soundly) separated from a global type in which they might appear embedded. As a further illustration, the following code is typable by assigning $\circ C C \mapsto 0$ to $f$ so that it captures the argument full behavior (and stores it in heap variable $a$ ) rather than borrowing it.

$$
\begin{aligned}
& \text { let } c=\text { new Coll }[] \text { in } \\
& \quad \text { var } a \text { in let } f=\lambda x . a:=x \text { in }((f c) ;(\text { a.init " } y "))
\end{aligned}
$$

We now consider some examples with concurrency. The parallel expression $\left(e_{1} \| e_{2}\right)$ clearly brings up the possibility of interference. The next couple of examples are safe, and type-check in our system

$$
\begin{aligned}
& \text { let } c=\text { newColl }[] \text { in } \\
& \quad((\text { c.init " } m y ") ;(\text { c.add } 1) ;(\text { c.scan } \| \text { c.scan })) \\
& \text { let } c=\text { newColl }[] \text { in let } f=\lambda x .(x . s c a n) \text { in } \\
& \quad((\text { c.init " } m y ") ;((f c) \| \text { c.scan }) ;(\text { c.getId } \|(\text { c.add } 2)) ;(f c))
\end{aligned}
$$

On the other hand, the code snippets

$$
\begin{aligned}
& \text { let } c=\text { newColl }[] \text { in }((\text { c.init "my" }) ;((\text { c.add } 1) \|(\text { c.scan })) \\
& \text { let } c=\text { newColl }[] \text { in } \\
& \quad \text { let } f=\lambda x .((x . a d d) \|(\text { c.add } 1)) \text { in }((\text { c.init "my" }) ;(f c))
\end{aligned}
$$

violate the intended behavioral separation constraints, and are rejected by the type system. In the last case, although the function $f$ may be given type $(a d d$ :nat $\mapsto 0) \mapsto 0$, the application $(f c)$ is not typable, since the type $C C$ cannot provide footprints for separately typing function and argument (no parallel separated add capabil- 
ities are available on $c$ ). On the other hand, the following similar looking code is safe and well-typed.

$$
\begin{aligned}
& \text { let } c=\text { newColl }[] \text { in } \\
& \quad \text { let } f=\lambda x .(x . s c a n \| \text { c.scan }) \text { in }((\text { c.init " } m y ") ;(f c))
\end{aligned}
$$

The form $\left(e_{1} \| e_{2}\right)$ is actually derived from primitive fork and wait thread-based concurrency constructs. Thread references are firstclass values in our language, created by the fork $(e)$ expression. The interesting operation on threads is to wait for their return value. Let

let $c=$ newColl [] in ((c.init "my");

$$
\text { var } a \text { in }(a:=\text { fork }(\text { c.scan }) ; \text { c.scan; wait }(a) ;(\text { c.add } 1)))
$$

This code is well-typed under the current typing assumptions for $c$, as enforced by the parallel and sequential frame conditions, since the footprints of the fork and wait expressions match the expectations of the global behavioral type. On the other hand,

$$
\begin{aligned}
& \text { let } c=\text { new Coll }[] \text { in }((\text { c.init "my" }) ; \\
& \quad \text { var } a \text { in }(a:=\text { fork }(\text { c.scan }) ;(\text { c.add } 1) ; \text { wait }(a)))
\end{aligned}
$$

is not well-typed under the same assumptions: it breaks the separation constraints required by the type of $c$. Such type requires c.scan and (c.add 1) to be sequentially separated, but overlapping may occur at runtime, causing unsafe interference.

As noticed before, it is not sound to assign to our collection a type allowing the add operation to be used concurrently with scan operations. That would violate the intended usage protocol of the internal state, causing a write/read race on heap variable $h d$. However, our language allows "critical regions" in the code to be sequentialized, and eventually typed by invariant-based separation. Invariant-based separation allows isolated behaviors to be repeatedly interleaved in the global behavior, as far as the associated invariant conditions, expressed by a conveniently chosen type assertion, are preserved. In our example, this could be achieved, e.g., by adding a new local heap variable inv to collection, and wrapping the uses of $h d$ in add and scan as follows

$$
\begin{aligned}
& a d d=\lambda e \cdot \operatorname{sync}(i n v)(\ldots) \\
& s c a n=\operatorname{var} s \text { in }(\operatorname{sync}(i n v) s:=h d ; \text { rec } L . \text { case } s \text { of } \ldots)
\end{aligned}
$$

To type this code, we associate an assertion $h d: r d($ ! $P$ Node $)$; var to the heap variable inv, which expresses an invariant condition protecting its footprint. Our type system is then able to assign to the concurrent collection the following type, which allows, after initialization, operations getId, add and scan to be unboundedly aliased, or shared by several active threads.

$$
C \triangleq(\text { init:str } \mapsto 0) ;(\text { getId:str } \mid \text { !scan:0| !add:nat } \rightarrow 0)
$$

Of course, the main novelty to highlight here is not the familiar reasoning technique for lock invariants, but the way our type discipline elegantly captures it. Even if based on a few fairly general principles, it can be effectively used to reason about safety properties of higher-order concurrent programs involving difficult to handle scenarios of aliasing and concurrency. We are not aware of related proposals, able to address the same set of (realistic) programming idioms, and based on a similarly general foundation, as we have achieved here. This paper makes the following contributions:

- We motivate and introduce the concept of behavioral separation as a general principle for disciplining interference in higherorder imperative concurrent programs.

- We present a behavioral separation type system for a $\lambda$-calculus

\begin{tabular}{|c|c|c|}
\hline$e, f \quad::=$ & $\begin{array}{l}x \\
\lambda x . e \\
e_{1} e_{2} \\
\text { let } x=e_{1} \text { in } e_{2} \\
\text { var } a \text { in } e \\
a:=v \\
a \\
{\left[l_{1}=e_{1}, \ldots\right]} \\
e . l \\
l(e) \\
\operatorname{case} e \text { of } l_{i}\left(x_{i}\right) \rightarrow e_{i} \\
\operatorname{rec}(X) e \\
X \\
\text { fork } e \\
\text { wait } e \\
\text { sync }(a) e \\
\text { sy }(a) e\end{array}$ & $\begin{array}{l}\text { (Variable) } \\
\text { (Abstraction) } \\
\text { (Application) } \\
\text { (Definition) } \\
\text { (Heap variable decl) } \\
\text { (Assignment) } \\
\text { (Dereference) } \\
\text { (Tupling) } \\
\text { (Selection) } \\
\text { (Variant) } \\
\text { (Conditional) } \\
\text { (Recursion) } \\
\text { (Recursion variable) } \\
\text { (New thread) } \\
\text { (Wait) } \\
\text { (Synchronized block) } \\
\text { (inSynchronized block) }\end{array}$ \\
\hline
\end{tabular}
with imperative and concurrency constructs. We show soundness of the system, proving type preservation under reduction (Theorems 4.3 and 4.5) and progress (Theorem 4.4).

- We illustrate, by means of many examples, how our type system, even if based on a small set of very general primitives, is already able to tackle fairly challenging program idioms.

$$
\begin{array}{rrll}
m, n, t \ldots \in & \Lambda & \text { (Names) } \\
x, y, z \ldots \in & \mathcal{V} & \text { (Variables) } \\
a, b, c \ldots \in & \Lambda \cup \mathcal{V} & \text { (Identifiers) } \\
l, s \ldots \in & \mathcal{L} & \text { (Labels) } \\
X, Y \ldots \in & \chi & & \text { (Expression Vars) }
\end{array}
$$

Figure 1. Programming Language.

\section{Programming Language}

Our programming language, presented in Figure 1, is a $\lambda$-calculus with mutable heap allocated variables, tuples, variants, and concurrency primitives. To keep it close to familiar high-level languages such as Java, we consider unstructured (fork/join) thread-oriented concurrency primitives, and a synchronization construct. Our language is fairly simple yet expressive enough to support challenging imperative higher-order concurrent programming idioms.

To formally define it, we assume given an infinite set of names $\Lambda$, an infinite set of variables $\mathcal{V}$, and an infinite set of method labels $\mathcal{L}$. Names in $\Lambda$ are used to identify threads and heap locations. For simplicity sake, we omit basic values, and literals for booleans or integers, their addition as primitives is straightforward.

The functional core includes abstraction $\lambda x . e$ and application $e_{1} e_{2}$, following call-by-value evaluation. The tuple expression $\left[l_{1}=e_{1}, \ldots\right]$ denotes a record collecting expressions $e_{i}$, each one qualified by the label $l_{i}$. As in [34], and without any loss of generality, we consider lazy tuples, where the expression $e_{i}$ is only evaluated after selection of the label $l_{i}$. Lazy tuples allow different qualifications of the same entity to be subject to different interference constraints, both along the time and space dimensions, and are convenient for encoding objects as tuples of "methods". The empty tuple [] is abbreviated by nil.

The let expression represents local definition and sequential composition: in let $x=e_{1}$ in $e_{2}$, the subexpression $e_{1}$ is executed first, its result bound to $x$, and only after $e_{2}$ gets evaluated. We abbreviate let $x=e_{1}$ in $e_{2}$ by $\left(e_{1} ; e_{2}\right)$ if $x$ is not free in $e_{2}$.

The construct $l(e)$ injects the value of expression $e$ into the variant label $l$. The case construct corresponds to a standard destructor for labeled sum types. The expression case $e$ of $l_{i}\left(x_{i}\right) \rightarrow e_{i}$ first evaluates $e$ to a variant value $l_{i}(v)$ (if this is not the case, execution will get stuck). Then, $v$ is bound to $x_{i}, e_{i}$ evaluated and its value returned as the result of the whole case expression.

Variable declaration var $a$ in $e$, variable access $v$, and assignment $a:=x$ are interpreted as usual. We adopt a simple form of assignment $a:=v$ where $v$ must be a value, and often use $a:=e$ as an abbreviation of let $x=e$ in $a:=x$. 
The expression fork $e$ spawns a new thread dedicated to the evaluation of expression $e$, and immediately returns the new thread identifier to the caller. Both the calling thread and the newly created one proceed execution concurrently. The expression wait $e$ suspends the caller until the thread resulting from evaluating $e$ terminates with some result (if ever). Such result will then be returned as the result of the wait expression.

Our language includes a simple synchronization primitive. The primitive relies on endowing each heap variable with a lock. Such locks are available for flexible use in programs, pretty much as object locks are used, e.g., in Java programs (either to lock the variable itself, or to protect any other relevant region of the state). At each moment, a lock may be either taken or free. The expression $\operatorname{sync}(n) e$ evaluates the expression $e$ in exclusion, using the lock associated to heap variable $n$ (cf. the Java synchronized block); only one thread may acquire the lock of $n$ in linear ("write") mode.

To track entry and exit of synchronization blocks in the operational semantics, we use the auxiliary construct $s y(n) e$. No occurrences of this construct, or of location or thread names are expected to appear in source programs, these elements belong to the runtime syntax of the full language, as shown in Figure 1.

The operational semantics of our programming language is defined by a reduction system using evaluation contexts. A state consists of a pair $h ; T$, where $h$ is a heap and $T$ is a multiset of threads. A reduction step has the form

$$
h ; T \rightarrow h^{\prime} ; T^{\prime} \quad\left(h ; T \text { reduces to } h^{\prime} ; T^{\prime}\right)
$$

expressing a computation step from state $h ; T$ to state $h^{\prime} ; T^{\prime}$. In any such step, new heap cells may be allocated, threads may be created, evolve, or terminate. Each thread in $T$ is represented by an element of the form $t\langle e\rangle$, where $t$ is the thread name (from $\Lambda$ ), and $e$ is the runtime expression under execution by the thread. We write $t\langle e\rangle \cdot T$ for the multiset union of $T$ and $t\langle e\rangle$. A heap $h$ is a mapping from heap locations (names) to values. Each heap binding $n_{k} \mapsto v$ also has an integer-valued counter $k$ associated, to be used as a semaphore, important to support the synchronization primitives. We write $h\left(n_{k} \mapsto v\right)$ to denote a heap such that $n_{k} \mapsto v \in h$, and $h\left[n_{k} \mapsto v\right]$ to denote the heap obtained from $h$ after storing $v$ at location $n$, with lock value $k$. We now introduce values $\mathcal{V}$ and evaluation contexts $\mathcal{E}$, given by

$$
\begin{array}{rlrl}
v, u \in \mathcal{V} & ::= & \lambda x . e\left|\left[l_{1}=e_{1}, \ldots\right]\right| l(v)|t| x \\
\mathcal{E} & ::= & \square \mid \text { let } x=\mathcal{E} \text { in } e|\mathcal{E} . l| \mathcal{E} e|v \mathcal{E}| l(\mathcal{E}) \\
& \quad \text { wait }(\mathcal{E})|s y(n) \mathcal{E}| \text { case } \mathcal{E} \text { of } \overline{l_{i}\left(x_{i}\right) \rightarrow e_{i}}
\end{array}
$$

A value in our language is either an abstraction, a tuple (including the empty tuple nil), a variant value, a thread name, or a variable. The rules defining the reduction relation are presented in Figure 2. We write $\{v / x\}$ for the capture avoiding substitution of $v$ for $x$, defined as expected. In all reduction rules, we denote by $t\langle e\rangle \cdot T$ a multiset that contains a thread $t\langle e\rangle$ and a rest $T$. Notice that there is no order assumed between elements in the thread multiset, so any thread may be (non-deterministically) scheduled at each reduction step. Most reduction rules are easy to interpret, and do not deserve much explanation. In rules (Red var) and (Red fork) the conditions $(\boldsymbol{\nu} n)$ and $(\boldsymbol{\nu} s)$ state that names $n$ and $s$ must be fresh in the respective left hand side.

Rules (Red sync*) rely on the integer-valued lock associated to the each heap location $n$. When the lock is zero, the lock is free. Rule (Red syncin) checks that the lock $k$ associated to $v$ is free, before decrementing it to -1 , and allowing execution to enter the critical region $e$ : the expression $s y(n) e$ signals that the execution of $e$ is taking place inside a critical region protected by the lock of $n$. The lock is released after the body of the $s y(n) e$ block reduces to a value $u$, in rule (Red syncout).
(Red rec)

$h ; t\langle\mathcal{E}[\operatorname{rec}(X) e]\rangle \cdot T \rightarrow h ; t\langle\mathcal{E}[e\{\operatorname{rec}(X) e / X\}]\rangle \cdot T$

(Red let)

$h ; t\langle\mathcal{E}[$ let $x=v$ in $e]\rangle \cdot T \rightarrow h ; t\langle\mathcal{E}[e\{v / x\}]\rangle \cdot T$

(Red beta)

$h ; t\langle\mathcal{E}[(\lambda x . e) v]\rangle \cdot T \rightarrow h ; t\langle\mathcal{E}[e\{v / x\}]\rangle \cdot T$

(Red sel)

$h ; t\left\langle\mathcal{E}\left[[\overline{l=e}] . l_{i}\right]\right\rangle \cdot T \rightarrow h ; t\left\langle\mathcal{E}\left[e_{i}\right]\right\rangle \cdot T$

(Red case)

$h ; t\left\langle\mathcal{E}\left[\right.\right.$ case $l_{i}(v)$ of $\left.\left.\overline{l(x) \rightarrow e}\right]\right\rangle \cdot T \rightarrow h ; t\left\langle\mathcal{E}\left[e_{i}\left\{v / x_{i}\right\}\right]\right\rangle \cdot T$

(Red var)

$h ; t\langle\mathcal{E}[\operatorname{var} a$ in $e]\rangle \cdot T \rightarrow h[n \mapsto \mathbf{n i l}] ; t\langle\mathcal{E}[e\{n / a\}]\rangle \cdot T \quad(\boldsymbol{\nu} n)$

(Red assign)

$h\left(n_{k} \mapsto v\right) ; t\langle\mathcal{E}[n:=u]\rangle \cdot T \rightarrow h\left[n_{k} \mapsto u\right] ; t\langle\mathcal{E}[\mathbf{n i l}]\rangle \cdot T$

(Red deref)

$h\left(n_{k} \mapsto v\right) ; t\langle\mathcal{E}[n]\rangle \cdot T \rightarrow h\left(n_{k} \mapsto v\right) ; t\langle\mathcal{E}[v]\rangle \cdot T$

(Red fork)

$h ; t\langle\mathcal{E}[$ fork $e]\rangle \cdot T \rightarrow h ; t\langle\mathcal{E}[s]\rangle \cdot s\langle e\rangle \cdot T$

(Red wait)

$h ; t\langle\mathcal{E}[$ wait $s]\rangle \cdot s\langle v\rangle \cdot T \rightarrow h ; t\langle\mathcal{E}[v]\rangle \cdot T$

(Red syncin)

$h\left(n_{0} \mapsto v\right) ; t\langle\mathcal{E}[\operatorname{sync}(n) e]\rangle \cdot T \rightarrow h\left[n_{-1} \mapsto v\right] ; t\langle\mathcal{E}[s y(n) e]\rangle \cdot T$

(Red syncout)

$h\left(n_{-1} \mapsto u\right) ; t\langle\mathcal{E}[s y(n) v]\rangle \cdot T \rightarrow h\left[n_{0} \mapsto u\right] ; t\langle\mathcal{E}[v]\rangle \cdot T$

Figure 2. Reduction.

\section{Type System}

In this section, we technically present our type system. As already discussed, types describe behavioral usages of values. We start by

\begin{tabular}{|c|c|c|}
\hline$T, U \quad::=$ & $\begin{array}{lr}0 & \text { (stop) } \\
T ; U & \text { (sequential) } \\
T \& U & \text { (intersection) } \\
\oplus l \in I l: T_{l} & \text { (sum) } \\
\circ T & \text { (isolated) } \\
\operatorname{rec}(X) T & \text { (recursion) }\end{array}$ & $\begin{array}{lr}T \mapsto V & \text { (function) } \\
T \mid U & \text { (parallel) } \\
l: T \quad \text { (qualification) } \\
! T & \text { (shared) } \\
\tau(T) & \text { (thread) } \\
X & \text { (recursion var) }\end{array}$ \\
\hline
\end{tabular}
systematically introduce each type operator, discussing on the way their basic algebraic properties and related subsumption laws.

DEFINITION 4.1 (Types). Type operators are given by

We assume given some primitive type constructors c, c $(U)$, such as str, nat, to represent basic data types, and var, $\operatorname{rd}(U)$, etc, to represent behavioral separation types for heap allocated variables. The stop type 0 types any value exposing no behavioral capability, in particular it types nil. The sequential type $T ; U$ asserts of a value that it can be safely used first according to type $T$, and only afterwards according to type $U$. The sequential type expresses behavioral separation along the temporal dimension. Sequential types induce a monoid with identity 0 in the type structure, expressed by

$$
U ;(V ; T)<:>(U ; V) ; T \quad U ; 0<:>U \quad 0 ; U<:>U
$$

The parallel type $T \mid U$ asserts of a value that it can be subject to two safe independent parallel usages, specified by type $T$ and type $U$ respectively. By "independent parallel usage" of a value we mean any form of sharing, arising not just in concurrent programs, but also in sequential programs, due to aliasing. The parallel type 
thus expresses behavioral separation along the spatial dimension. It builds on the fundamental idea of separation (cf. separation logic [33]), but focusing on the independence of usage behaviors, as perceived from a "client" viewpoint, rather than on the disjointness of underlying resources. To highlight this understanding of $T \mid U$, we refrain from using the notation $T * U$. A key insight on $(-\mid-)$ is that behaviors typed by parallel separation do not interfere in unsafe ways, even if they rely on shared underlying resources.

An usage of type $U \mid V$ only concludes when both $U$ and $V$ conclude. In a type such as $(U \mid V) ; T$, the usage $T$ is only available when $(U \mid V)$ conclude. So our type language provides an abstract way of splitting "permissions", without using explicit fractions (cf. [9]). Parallel types induce a commutative monoid with identity 0

$$
U|(V \mid T)<:>(U \mid V)| T \quad U|V<:>V| U \quad U \mid 0<:>U
$$

Sequential and parallel composition are related by the exchange law $[4,17]$, the following causality preserving distribution principle

$$
(A ; C) \mid(B ; D)<:(A \mid B) ;(C \mid D)
$$

A special case is the familiar interleaving law $U \mid V<$ : $V ; U$.

The recursive type $\operatorname{rec}(X) . A$, where $X$ must occur guarded in $A$, is interpreted in a standard (co-inductive) sense.

The shared type !T asserts of a value that it can be safely subject to an unbounded number of parallel separated usages (cf. $(-\mid-)$ ), each one specified by type $T$. In particular, it may be unboundedly aliased at type $T$. The following laws hold for the type $! T$

$$
\begin{gathered}
! U<: U \quad ! U<: ! ! U \quad 0<: ! 0 \quad ! U|!|<<!(U \mid V) \\
! U<: 0 \quad ! U<: ! U \mid ! U
\end{gathered}
$$

Notice that !(-) satisfies the fundamental co-monadic laws for the linear logic exponential; so our notation highlights the connection.

The function type $T \mapsto V$ asserts of a value that it can be safely used (once) as a function that when given as argument a value of type $T$, exercises on it a usage of type $T$, and returns a result of type $V$. Type $U \mapsto V$ is adjoint to $U \mid V$, so that the behavioral separation interpretation ensures the intended safety property: no unsafe interference can arise even if function and argument share state-full resources, since they are behaviorally separated. So $U \mapsto V$ is a type for functions that do not unsafely interfere with their arguments, as aimed in the sharing interpretation of $-\star$ in [29], but does not completely forbid sharing to enforce safety of interference. Moreover, unlike in the linear logic interpretation of the arrow $(U \multimap V)$, a function of type $U \mapsto V$ can use its parameter more than once, as long as it globally respects the behavioral type $U$.

The isolated type $\circ T$ asserts of a value that it may be used as specified by the type $T$, but, more crucially, that such usage is fully isolated, not subject to any external (global) constraints. A value of type $\circ T$ is completely separated (in terms of behavior) from the rest of the "world". In particular, $\circ T$ says that the usage $T$ is not borrowed from some larger computation. We may see a value of type $\circ T$ as offering a self-contained suspended behavior of type $T$, that may be used at any future step in the computation. No liveness commitments are imposed on client code to use a value of type $\circ T$, unless it actually starts to use it at type $T$. In particular, a value of type $\circ T$ may be safely dropped out, since nothing causally depends on it: a safe use for a value of type $\circ T$ is not to use it at all. Moreover, since nothing can causally depend on a value of type $\circ T$, we expect the law $(\circ U) ; V<:(\circ U) \mid V$ to hold. We also have

$$
\begin{gathered}
0<: \circ 0 \quad \circ A \mid \circ B<: \circ(A \mid B) \quad \circ A<: A \quad \circ A<: \circ \circ A \\
\circ A<: 0 \quad ! \circ A<: \circ ! A \quad(\circ A \mid B) ; C<: \circ A \mid(B ; C)
\end{gathered}
$$

The first five laws express familiar algebraic principles (cf. the basic laws for $!(-))$. The last two laws are proper to $\circ(-)$. In particular, the last one expresses the key property of $\circ(-)$, global behavioral isolation: a behavior of type $\circ T$ is isolated, and can be freely used anytime, concurrently with anything. No other behavior can causally depend from a behavior of type $\circ T$. By the exchange law, we may derive the "postponing" principle $(\circ A) ; B<: B ;(\circ A)$.

The (linear) intersection type $U \& V$ asserts of a value that it may be safely used according to type $U$ and according to type $V$. The client code using such a value can therefore freely decide to pick either the $U$ or the $V$ behavior (but not both, since we exploit a linear interpretation of \&). The following basic laws hold.

$$
U \& V<: U \quad U \& V<: V \quad U<: U \& U
$$

The qualified type $l: T$ asserts of a value that it offers a usage of type $T$ under the label $l$. It describes a label selection capability of a tuple, classifying the usage type of the value in field $l$. Following [35], general tuple types may be defined by combining qualified types with other type constructors, e.g. $l_{1}: T_{1} \& \ldots \& l_{n}: T_{n}$.

The sum type $\oplus_{l \in i} l_{i}: T_{i}$ asserts of a value that it is a labeled value that can be used according to type $T_{i}$ if it is labeled with $l_{i}$. Client code using such a value must branch on the possible labels, before actually using the selected behavior. The sum type thus corresponds to a standard labeled disjoint union, useful to describe variants or options. We do not assume specific subtyping principles for sum types. We abbreviate (NULL:0 $\oplus$ NODE: $U$ ) by Opt $(U)$.

The thread type $\tau(T)$ asserts of a value that it references a running thread that upon termination returns a value of type $T$.

Types for heap allocated variables are conveniently described in our system by specific primitives, expressing usage, write and read capabilities. The type of a freshly allocated heap variable is var. The type var denotes the generic heap variable usage protocol, and is axiomatized by several subtyping laws, presented in Section 4.1.

As previously discussed, a type classifies a single value. In order to type program expressions, which may use in general several different values, we introduce a notion of type assertion. A type assertion corresponds to the usual notion of type environment, assigning types to the various free identifiers in a program. However, our type assertions finely describe behavioral dependencies between the several identifiers in its domain, by placing basic type assignments of the form $x: T$ embedded in a larger global type.

DEFINITION 4.2. Type assertions are given by

$$
A, B:=x: T|A ; B| A|B| A \& B|! A| \circ A|X| \operatorname{rec}(X) A
$$

For an example, under the assumptions expressed by the type assertion $(f: U \mapsto V ; y: U) \mid z: U$, the function $f$ can be applied to $z$ but not to $y$, so $(f z)$ is well typed but $(f y)$ is not, since the behavior $y: U$ is only available after $f: U \mapsto V$ is used.

We denote by $\operatorname{Dom}(A)$ the (finite) set of variables appearing in a type assertion $A$. If $A$ has a singleton domain $\{x\}$ (that is, refers to a single variable $x$ ), we implicitly identify it with the singular assertion $x:(A)_{x}$ where the type $(A)_{x}$ is given by

$$
\begin{array}{ll}
(x: T)_{x}=T & (A ; B)_{x}=(A)_{x} ;(B)_{x} \\
(A \mid B)_{x}=(A)_{x} \mid(B)_{x} & (A \& B)_{x}=(A)_{x} \&(B)_{x} \\
(! A)_{x}=!(A)_{x} & (\circ A)_{x}=\circ(A)_{x} \\
(X)_{x}=X & (\operatorname{rec}(X) A)_{x}=\operatorname{rec}(X)(A)_{x}
\end{array}
$$

Therefore, we identify, e.g., $x:(u p ; d n)$ with $(x: u p) ;(x: d n)$, and $\operatorname{rec}(X)((x: u p \mid x: d n) ; X)$ with $x: \operatorname{rec}(X)((u p \mid d n) ; X)$.

We define type assertion contexts $\mathcal{A}[-], \mathcal{E}[-]$ to be the one hole syntactic contexts associated to type assertions. We also consider active assertion contexts, where the hole occurs unguarded, defined

$$
\mathcal{E}[-]::=\square|\mathcal{E}[-] ; A| \mathcal{E}[-]|A| A \mid \mathcal{E}[-]
$$

\subsection{Subtyping}

Type assertions are related by a subtyping relation. We write $A$ <: $B$ to state that $A$ is a subtype of $B$, meaning that the usage behavior $B$ is subsumed by usage behavior $A$. Intuitively, $A<$ : $B$ means that 


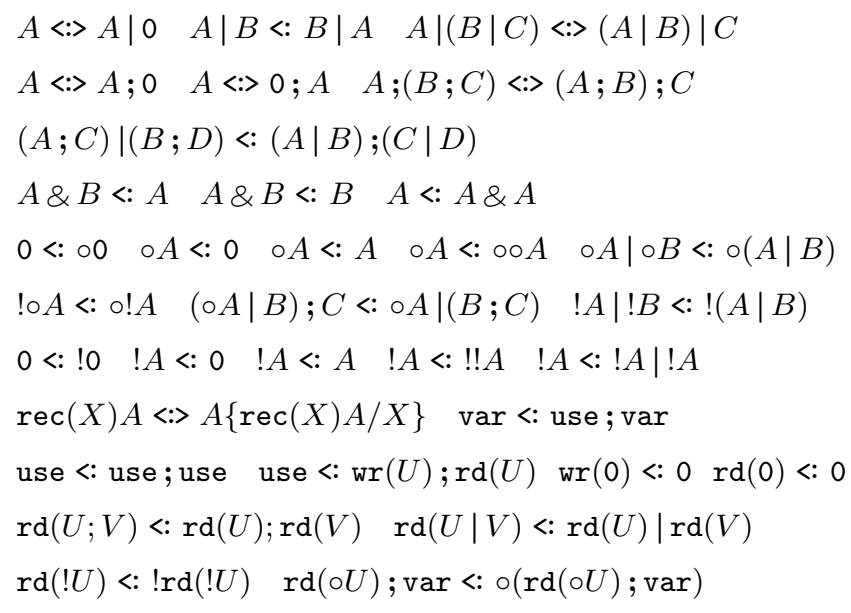

Figure 3. Subtyping.

if some value may be safely used according to $A$ then it may also be safely used according to $B$.

\section{$A<: B \quad(A$ is a subtype of $B)$}

Notice that subtyping also apply to types by letting $U<: V$ if and only if $x: U<: x: V$. Subtyping axioms, defined in Figure 3, express the basic algebraic laws of the type operators discussed above. We abbreviate by $A<:>B$ the fact that $A<: B$ and $B<$ : $A$. To save space, we abbreviate rules of the form $x: U<: x: V$ by $U<: V$, and omit subtyping congruence rules. All type operators satisfy the expected (covariant) subtyping congruence principles, with some exceptions, e.g., the arrow $U \mapsto V$, which is contravariant in the domain $U$, and $\operatorname{wr}(V)$, which is contravariant on $V$ (see Appendix).

Particularly interesting are the axioms defining the var behavior. We may derive var <: ovar: clearly a fresh heap allocated variable offers an isolated behavior. The first axioms state that a variable can be subject to an unbounded number of uses, each use composed by a write and a read phase. Other axioms specify how the reading phase may be behaviorally separated, depending on the type of the stored value. For example, the axiom for $\operatorname{rd}(U \mid V)$ says that if a value of type $U \mid V$ can be read from the variable, then the variable can also be subject to independent reading at types $U$ and $V$. This point is extremely important: e.g., a heap variable may be shared or aliased, only if the stored value also may be. Notice that the axiom for use, allowing a different type $U$ to be picked at different unfoldings of var, naturally support strong updates [2] (updating a heap allocated variable to hold values of unrelated types at different points in time).

\subsection{Typing}

Type judgments of our system have the form

$$
A \vdash_{z} e:: B \quad \text { (e types from } A \text { to } z \text { in } B \text { ) }
$$

where $A$ and $B$ are type assertions, $e$ is an expression, and the index $z$ is a variable. We refer to $A$ as the pre-condition, and to $B$ as the post-condition of the typing judgment. The behavioral type of (the value of) $e$, as determined by the type system, appears embedded in assertion $B$. The variable $z$ stands for such a value, and can only occur free in $B$ (not in $A$ or $e$ ). This idea of scoping the return value $z$ over the post-condition already appears in the Hoare triple type of [28], although here the type of $z$ cannot be given apart from $B$.

For an example, consider

$$
a: \text { use } \vdash_{z}(\lambda x . a:=x):: z: \circ U \mapsto 0 ; a: \operatorname{rd}(\circ U)
$$

This judgment asserts that evaluating the expression $(\lambda x . a:=x)$ in a state providing $a$ :use returns a functional value (identified by $z$ in the post-condition) that must be used exactly once before the heap variable $a$ can be read. We now progressively present the several rules of our type system, discussing each one on the way.

\subsubsection{Structural Rules}

The identity axiom

$$
x: U \vdash_{z} x:: z: U(I d)
$$

asserts that access to the identifier $x$ simply returns the associated value, usable according to the type in the pre-condition (N.B: $(I d)$ has the proviso that $U$ is not an heap variable type: typing rules for heap variable dereference are given below). The type system includes four other structural rules. A crucial one is subtyping $(S u b)$, which embeds into typing the basic subsumption principles. It allows assertions in type rules to be considered up to $\langle:\rangle$, and plays a role similar to the consequence rule in Hoare logics.

$$
\frac{A<: A^{\prime} \quad A^{\prime} \vdash_{x} e:: B^{\prime} \quad B^{\prime}<: B}{A \vdash_{x} e:: B}(S u b)
$$

The rule for let corresponds to cut ( $x$ not free in the conclusion)

$$
\frac{A \vdash_{x} e_{1}:: B \quad B \vdash_{y} e_{2}:: C}{A \vdash_{y} \text { let } x=e_{1} \text { in } e_{2}:: C}(\text { Let })
$$

The following parallel and sequential structural rules express basic "frame" principles. Rule (Par) allows the footprint of an expression to be enlarged along the spatial dimension, while rule $(\mathrm{Seq})$ allows the footprint to be enlarged along the temporal dimension.

$$
\frac{A \vdash_{x} e:: B}{A\left|C \vdash_{x} e:: B\right| C}(\text { Par }) \quad \frac{A \vdash_{x} e:: B}{A ; C \vdash_{x} e:: B ; C}(\text { Seq })
$$

Given these two rules, the following "deep" frame rule is admissible for any active type assertion context $\mathcal{E}[-]$.

$$
\frac{A \vdash_{x} e:: B}{\mathcal{E}[A] \vdash_{x} e:: \mathcal{E}[B]}(\text { Frame })
$$

\subsubsection{Functional Type}

We have the following typing rules for the $\lambda$-calculus core.

$$
\begin{gathered}
\frac{A \mid x: U \vdash_{y} e:: y: T}{A \vdash_{z} \lambda x . e:: z: U \mapsto T}(V A b s) \\
\frac{A \vdash_{z} e_{1}:: z: U \mapsto_{T} \quad B \vdash_{x} e_{2}:: x: U}{A \mid B \vdash_{y} e_{1} e_{2}:: y: T}(A p p)
\end{gathered}
$$

These rules are similar to the arrow rules in linear or bunched [29] type systems, even if our semantics for $\mapsto$ is different. Given the interpretation of $A \mid B,(A p p)$ ensures that functions do not interfere with their arguments unsafely upon application. Notice that the type of the argument $x$ is left 0 in the postcondition of the premise of $(V A b s)$, forcing the function body to fully exercise the behavior $U$ of its parameter, consistently with the "argument-borrowing" semantics of our functional type. The type of an "argumentcapturing" function may be rendered $(\circ U) \mapsto V$, and the type of a function that can safely share the behavior of its argument with its own behavior may specified $(! U) \mapsto V$. Behavioral separation types allow many fine-grained variations of functional behavior to be specified (e.g., ! $(U \mapsto V)$ - a shareable function; $(U \mapsto V)^{*}$ - a non shareable but repeatedly usable function, etc). Notice that in our typed language (as, e.g., in the monadic $\lambda$-calculus) one cannot encode the let by application and abstraction.

\subsubsection{Tuple Type}

The rules for tuples and field selection have the expected form.

$$
\frac{A \vdash_{x} e:: x: U}{A \vdash_{z}[\ldots l=e \ldots]:: z: l: U}(\text { Tuple }) \quad \frac{A \vdash_{z} e:: z: l: T}{A \vdash_{x} e . l:: x: T}(S e l)
$$


Recall that field contents of tuples are evaluated lazily, as in [34], so (Tuple) allows a single field to be type checked. As explained above, richer behavioral separation types for multi-field records may be expressed using the various type constructors available.

\subsubsection{Intersection Type}

We include as primitive the introduction rule $A n d$.

$$
\frac{A \vdash_{y} e:: B \quad A \vdash_{y} e:: C}{A \vdash_{y} e:: B \& C}(A n d) \quad \frac{A \vdash_{y} e:: B_{1} \& B_{2}}{A \vdash_{y} e:: B_{i}}(A n d E)
$$

Technically, we choose to absorb the elimination principles for intersection in the subtyping relation (e.g., $A \& B<: A$ ). However, familiar elimination rules $A n d E$ are admissible (using $(S u b)$ ).

\subsubsection{Behavioral-Separation Types}

Structured behavioral-separation usages are assigned to basic values (abstractions, tuples) by the following type rules:

$$
\begin{gathered}
0 \vdash_{y} v:: 0 \text { (VStop) } \quad \frac{A \vdash_{y} v:: C \quad B \vdash_{y} v:: D}{A ; B \vdash_{y} v:: C ; D}(\text { VSeq }) \\
\frac{! A_{1}|\ldots| ! A_{n} \vdash_{x} v:: B}{! A_{1}|\ldots| ! A_{n} \vdash_{x} v:: ! B}(\text { VShr }) \frac{A \vdash_{y} v:: C B \vdash_{y} v:: D}{A\left|B \vdash_{y} v:: C\right| D}(\text { VPar })
\end{gathered}
$$

Rule ( $V S h r)$ expresses that a value can be subject to any number of shared usages, if it only relies on resources which may also be safely used by any number of shared usages. Interestingly, these rules allow values to satisfy crisper frame principles than the structural rules in Section 4.2.1, which apply to general expressions. For example, the following "fat" identity axiom and left-sequential frame rule turn out to be admissible for values, even if the corresponding principles are not sound for arbitrary expressions.

$$
A \vdash_{y} v:: A(V I d) \quad \frac{B \vdash_{y} v:: C}{A ; B \vdash_{y} v:: A ; C} \text { (VLPar) }
$$

\subsubsection{Isolated Type}

The rule (Iso) assigns to the postcondition of an expression an isolated type if it only depends on values of isolated type.

$$
\frac{\circ A_{1}|\ldots| \circ A_{n} \vdash_{x} e:: B}{\circ A_{1}|\ldots| \circ A_{n} \vdash_{x} e:: \circ B} \text { (Iso) }
$$

The type rules for $! A$ and $\circ A$ are therefore similar, and express the basic comonadic principle associated to these type constructors (cf. the introduction rule for ! in intuitionistic linear logic), even if their meaning is quite different (sharing versus isolation).

A remarkable property of any type $T$ of the form !o $U$ is that $T<:>T \mid T$ and $T<:>\circ T$, so that $T$ is both shared and isolated.

\subsubsection{Sum Type}

Sum types are also handled by familiar looking typing rules.

$$
\begin{gathered}
\frac{A \vdash_{y} e_{c}:: y: \oplus_{l \in I} l: T_{l} \quad x_{i}: T_{i} \mid B \vdash_{z} e_{i}:: C}{A \mid B \vdash_{z} \text { case } e_{c} \text { of } l(x) \rightarrow e:: C} \text { (Case) } \\
\frac{A \vdash_{z} e:: z: T_{i}}{A \vdash_{z} l_{i}(e):: z: \oplus_{l \in I} l: T_{l}} \text { (Option) }
\end{gathered}
$$

As for function application, the type rule for case ensures that the matched value is separated from the corresponding case branch, so to avoid unsafe interference.

\subsubsection{Heap Variable Types}

We have already explained how heap allocated variables are modeled in our system as special values, subject to a specific usage protocol defined by certain subtyping axioms. The type rule for a variable declaration types the body under the assumption of a separated complete protocol for the new variable, specified by var.

$$
\frac{a: \operatorname{var} \mid A \vdash_{x} e:: C}{A \vdash_{x} \text { var } a \text { in } e:: C}(\text { Var })
$$

Rules for dereference and assignment are more interesting. We consider two typing rules for dereference, and two typing rules for assignment. The alternative typings express boundary cases on usage of the variable protocol, which are not naturally captured by a single typing rule. We distinguish between reading just a "piece" of the behavior stored in the variable $(R d V B)$, from reading the whole remaining stored behavior $(R d V F)$.

$$
\begin{gathered}
a: \operatorname{rd}(U) \vdash_{x} a:: x: U(R d V B) \\
a: \operatorname{rd}(U) ; \text { use } \vdash_{x} a:: x: U \mid a: \text { use }(R d V F)
\end{gathered}
$$

Rule $(R d V F)$ states that even if the precondition states that the next use of variable $a$ is guarded by a read usage $\operatorname{rd}(U)$, the variable content $x: U$ is separated of the residual variable behavior $a$ :use, specifying an "empty" variable. Rule $(R d V F)$ expresses an important invariant ensured by the type system: the behavior stored in any heap variable is always separated from the continuation behavior of the variable object itself (after all of its content gets read off). So the postcondition in the conclusion of ( $R d V F)$ always holds, even if the type of the heap variable content is not explicitly declared as isolated (not of the form $\circ U$ ).

We also have two rules for assignment, depending on weather the behavior stored in the heap variable is isolated or borrowed.

$$
\begin{gathered}
\frac{A \vdash_{z} v:: z: \circ U \mid a: \operatorname{wr}(\circ U)}{A \vdash_{z} a:=v:: 0}(W r V F) \\
\frac{A \vdash_{z} v:: z: U \mid a: \text { use }}{A \vdash_{z} a:=v:: a: \operatorname{rd}(U)}(W r V B)
\end{gathered}
$$

In both rules, the stored value is required to be parallel separated from the heap variable, which must be in a ready-for-write state. Rule $(W r V F)$ handles the case in which the value behavior to be stored is isolated. Here, we only require the write capability, as the stored value may be used anytime later. Rule $(W r V B)$ handles the case in which the value behavior to be stored may be not isolated. In this case, one must ensure that all associated reads will happen before any sequential continuation of $z: U$, so a whole use is required in the premise, leaving the associated read usage active in the post-condition. It is interesting to see why a rule as $(W r V F)$, but considering a non-isolated type for the stored value, would not be sound. Let us consider a simple counterexample.

$$
\begin{aligned}
& r: U\left|a: \operatorname{wr}(U) \vdash_{x} r:: x: U\right| a: \operatorname{wr}(U) \\
& r: U \mid a: \operatorname{wr}(U) \vdash_{x} a:=r:: 0 \\
& r: U ; V \mid a: \operatorname{wr}(U) \vdash_{x} a:=r:: r: V \\
& r: U ; V \mid a: \operatorname{wr}(U) ; \operatorname{rd}(U) \vdash_{x} a:=r:: r: V ; a: \operatorname{rd}(U) \\
& r: U ; V \mid a: \text { use } \vdash_{x} a:=r:: r: V ; a: \operatorname{rd}(U)
\end{aligned}
$$

This candidate derivation states that after executing $a:=r$ the behavior $r: V$ is available before reading the variable $a$, thus violating the requirement that $r$ must be used as $U ; V$. A correct typing is:

$$
\begin{aligned}
& r: U \mid a: \text { use } \vdash_{x} r:: x: U \mid a: \text { use } \\
& r: U \mid a: \text { use } \vdash_{x} a:=r:: a: \operatorname{rd}(U) \\
& r: U ; V \mid a: \text { use } \vdash_{x} a:=r:: a: \operatorname{rd}(U) ; r: V
\end{aligned}
$$

Typing rules for assignment require separation between heap variable and stored value. This may suggest that typing of circular chains of references through the heap may be difficult, if not impossible. Although it is clear that linear behaviors cannot refer circularly to themselves, that is not the case for general behavioral separation types: some safe circular chains may still support separated behaviors, due to the presence of qualified tuples, or just 
because of sharing (including invariant based separation). We illustrate this point in Section 4.5, by typing a version of Landin's knot. The following rules are admissible (using the abbreviation $a:=e$ for let $x=e$ in $a:=x$, assuming $\mathcal{E}[-]$ an active type context).

$$
\frac{A \vdash_{z} e:: z: \circ U \mid a: w r(\circ U)}{A \vdash_{x} a:=e:: 0} \quad \frac{A \vdash_{z} e:: \mathcal{E}[z: U] \mid a: \text { use }}{A \vdash_{x} a:=e:: \mathcal{E}[a: \operatorname{rd}(U)]}
$$

The rules of the basic type system are shown in Figure 4. We avoid introducing here rules for recursion and recursive types whose treatment require the addition of extra information to typing judgments; details may be found in the Appendix. For clarity's sake, we present the type system in two steps, first without synchronization constructs, which is extended in Section 4.5 to the full language.

\subsection{Typing the Collection Implementation}

We get back to the running example in the Introduction. We have intuitively argued that a collection value may be assigned the type $\circ C C$, and the function newColl the type $0 \mapsto \circ C C$. We now discuss how such type is actually validated by our type system. We first type the list nodes. Consider the following abbreviations

$$
\begin{aligned}
& \text { InitNode } \triangleq \operatorname{setElt}:(\text { nat } \mapsto 0) ; \text { setNext:(!oPNode } \mapsto 0) \\
& \text { INode } \triangleq \text { !getNext:PNode|!getElt:nat } \\
& \text { Node } \triangleq \text { InitNode } ; \text { ! INode } \quad \text { PNode } \triangleq \text { !opt (INode) }
\end{aligned}
$$

The type PNode defines the behavior of a pointer to a list of (initialized) nodes, as created by the function newNode. We use option types to type list pointers: either the "null" pointer, tagged $\operatorname{NULL}(-)$ or a value of type INode, tagged by $\operatorname{NODE}(-)$.

Directed by rules VSeq, VShr and Tuple, the system assigns type Node to the tuple $[$ setElt $=\cdots]$ by checking that it can be subject to the given behavioral separation usage, while safely using its local resources (the variables next and elt). The variable elt gets assigned type wr(nat) ; !rd(nat): it is written just once (in the operation setElt), and available for shared reading from then on. Notice that var <: $w r(n a t) ; \operatorname{lrd}($ nat $)$, since nat is assumed shared (nat <: !nat). Variable next is typed wr $(\circ P N o d e) ; \operatorname{lrd}(P N o d e) ;$ var. After initialization (execution of the behavior InitNode), the node type evolves to !oINode; only operations getNext and getElt are available, usable by an unbounded number of aliases or concurrent clients. The shared behavior INode of the list nodes supports sharing of the linked list and allows the type !scan:0 to be assigned within $C C$. Recall

$$
C C \triangleq(\text { init: } \text { str } \mapsto 0) ;\left(\text { getId:str } \mid(! \text { scan }: 0 ; \text { add:nat } \mapsto 0)^{*}\right)
$$

Checking newCollection against type $0 \mapsto \circ C C$, involves verifying that the "object" tuple $[$ init $=\ldots]$ representing a collection can be safely subject to the behavioral separation usage specified by $C C$. After init, the variable $h d$ is assigned type $\operatorname{rd}(\circ P N o d e)$; var. This type is kept invariant between iterated executions of the add and scan operations.

It is particularly interesting to see how the scan operation is typed. A sequentially separated prefix PNode is borrowed from $h d$ to the local heap variable $s,(\circ P N o d e<:$ PNode; o PNode). After typing the assignment $s:=h d$, the type assertion is

$$
s:(\operatorname{rd}(\text { PNode }) ; \operatorname{var}) ; h d:(\operatorname{rd}(\circ \text { PNode }) ; \operatorname{var})
$$

which by subtyping (var <: ovar and postponing) leads to

$$
s: r d(P N o d e) ;(h d:(\operatorname{rd}(\circ P N o d e) ; \operatorname{var}) \mid s: v a r)
$$

where PNode is the behavior of $s$ actually used up by the various loop iterations. After typing the whole loop, the type assertion is

$$
h d:(\operatorname{rd}(\circ P N o d e) ; \operatorname{var}) \mid s: \text { var }
$$

which after closing the scope of $s$ (removing $s$ :var), gets us back to the invariant $h d: r d(\circ P N o d e)$; var.

\subsection{Type Preservation and Progress}

We now state the main correctness results for the basic type system, namely the subject reduction property and progress for welltyped programs. Type preservation and progress ensure that in a well typed program all values are properly used according to their assigned behavioral-separation types. In particular, given the structure of types assigned to variables, no write/write or read/write races while writing to heap variables are possible (it is not the case that use <: $(\operatorname{wr}(U) \mid \operatorname{wr}(V)) ; T$ or use <: $(\operatorname{rd}(U) \mid \operatorname{wr}(V)) ; T)$.

We first introduce a notion of typing for runtime configurations $h ; S$ defined by the following rules

$$
\begin{gathered}
\emptyset ; \emptyset \triangleright 0(E) \quad \frac{h ; S \triangleright \mathcal{E}[C] \quad C \vdash_{x} e:: x: T}{h ; S \cdot t\langle e\rangle \triangleright \mathcal{E}[t: \tau(T)]}(T) \\
\frac{h ; S \triangleright A \quad A<: B}{h ; S \triangleright B}(S) \quad \frac{h ; S \triangleright A \quad A \vdash_{x} v:: B}{h, n \mapsto v ; S \triangleright B\{x / n: \operatorname{var}\}}(
\end{gathered}
$$

In rule $(T), \mathcal{E}$ is an active type assertion context. The notation $B\{x / n$ :var $\}$ (with $n$ fresh in $B$ ) represents an update to assertion $B$ in which the behavior pieces assigned to $x \in \operatorname{Dom}(B)$ are substituted in place by reads to a new heap variable $n$ : essentially, all occurrences of the form $x: U$ in type $B$ are replaced by $n: \operatorname{rd}(U)$ and a $n$ :var is inserted in sequential and linear position relative to all $n: \operatorname{rd}(U)$ 's. We can state our type preservation result.

THEOREM 4.3. If $h ; S \triangleright A$ and $h ; S \rightarrow h^{\prime} ; S^{\prime}$ then $h^{\prime} ; S^{\prime} \triangleright A$.

An expression $e$ is live, noted live $(e)$, if it is not a value. A set $S$ of threads is live, noted live $(S)$ if there is some thread $t\langle e\rangle$ in $S$ such that live $(e)$. We can then prove

THEOREM 4.4. If $h ; S \triangleright A$ and live $(S)$ then $h ; S \rightarrow h^{\prime} ; S^{\prime}$.

Detailed proofs and definitions are given in the Appendix.

\subsection{Invariant-based Separation}

We now extend the basic type system to cover the full core language with sync $(a) e$ blocks, and invariant-based reasoning. As explained in Section 3, each heap variable is equipped with an associated lock (pretty much like a Java object is). To each lock, a resource invariant, expressed by an isolated typing assertion, is associated for verification purposes. We only accept for lock invariant a heap assertion $R$ such that $R<: \circ R$ (let us call "heap assertion" any type assertion that just refers to heap variable types - var, use, etc). Handling lock invariants requires some additional structure in our type system: we add to typing judgments an invariant mapping $\iota$, that associates to heap location locks their invariants:

\section{$A \vdash_{z}^{\iota} e:: B \quad$ (e types from $A$ to $z$ in $B$ under $\iota$ )}

The invariant mapping is propagated untouched by all typing rules, except in the new rule for variable declaration, which may introduce a lock invariant, and in the rules for $\operatorname{sync}(n) e$ and $s y(n) e$, which make use the lock invariant associated to heap location $n$ :

$$
\begin{gathered}
\frac{\iota(a)\left|A \vdash_{x}^{\iota \backslash a} e:: \iota(a)\right| B}{A \vdash_{x}^{\iota} \operatorname{sync}(a) e:: B}(\text { Sync }) \frac{A \vdash_{x}^{\iota \backslash a} e:: \iota(a) \mid B}{A \vdash_{x}^{\iota} s y(a) e:: B}(S y) \\
\frac{A<: B|R \quad a: \operatorname{var}| B \vdash_{x}^{\iota\{R / a\}} e:: C}{A \vdash_{x}^{\iota} \text { var } a \text { in } e:: C}(\text { Var })
\end{gathered}
$$

Without loss of generality, we assume that the invariant associated to some heap variable's lock does not talk about the variable itself, but only about other heap variables in scope. Consider the code snippet describing an "atomic" variable.

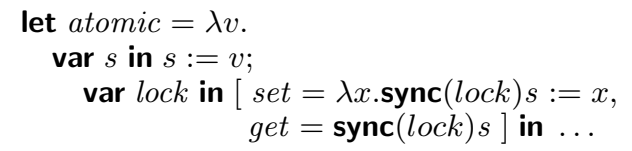




$$
\begin{aligned}
& 0 \vdash_{y} v:: 0 \text { (VStop) } \quad x: U \vdash_{y} x:: y: U(I d) \quad \frac{\circ A_{1}|\ldots| \circ A_{n} \vdash_{x} e:: B}{\circ A_{1}|\ldots| \circ A_{n} \vdash_{x} e:: \circ B} \text { (Iso) } \frac{! A_{1}|\ldots| ! A_{n} \vdash_{x} v:: B}{! A_{1}|\ldots| ! A_{n} \vdash_{x} v:: ! B} \text { (VShr) } \\
& \frac{A<: A^{\prime} \quad A^{\prime} \vdash_{x} e:: B^{\prime} \quad B^{\prime}<: B}{A \vdash_{x} e:: B}\left(\text { Sub) } \frac{A \vdash_{y} e:: B \quad A \vdash_{y} e:: C}{A \vdash_{y} e:: B \& C}(A n d) \frac{A \vdash_{x} e_{1}:: B \quad B \vdash_{y} e_{2}:: C}{A \vdash_{y} \text { let } x=e_{1} \text { in } e_{2}:: C}\right. \text { (Let) } \\
& \frac{A \vdash_{x} e:: B}{A ; C \vdash_{x} e:: B ; C}(S e q) \quad \frac{A \vdash_{x} e:: B}{A\left|C \vdash_{x} e:: B\right| C}(\text { Par }) \quad \frac{A \vdash_{x} e_{1}:: x: U \mapsto T \quad B \vdash_{y} e_{2}:: y: U}{A \mid B \vdash_{z} e_{1} e_{2}:: z: T}(\text { App }) \\
& \frac{A \vdash_{x} e:: x: U}{A \vdash_{x}[\ldots l=e \ldots]:: x: l: U}(\text { Tuple }) \quad \frac{A \vdash_{x} e:: x: l: T}{A \vdash_{x} e . l:: x: T}(\text { Sel }) \quad \frac{A \mid x: U \vdash_{z} e:: z: T}{A \vdash_{z} \lambda x \cdot e:: z: U \mapsto T} \text { (VAbs) } \\
& \frac{A \vdash_{y} e::\left(y: \oplus_{l \in I} l: T_{l}\right) \quad x_{l}: T_{l} \mid B \vdash_{z} e_{l}:: C}{A \mid B \vdash_{z} \text { case } e \text { of } \overline{l(\vec{x}) \rightarrow e}:: C} \text { (Case) } \frac{A \vdash_{z} e:: z: T_{m} \quad(m \in I)}{A \vdash_{z} l_{m}(e):: z: \oplus_{l \in I} l: T_{l}} \text { (Option) } \\
& \frac{A \vdash_{y} v:: C \quad B \vdash_{y} v:: D}{A ; B \vdash_{y} v:: C ; D}(\text { VSeq }) \quad \frac{A \vdash_{y} v:: C \quad B \vdash_{y} v:: D}{A\left|B \vdash_{y} v:: C\right| D}(\text { VPar }) \\
& \frac{a: \operatorname{var} \mid A \vdash_{x} e:: C}{A \vdash_{x} \operatorname{var} a \operatorname{in} e:: C}(\operatorname{Var}) \quad \frac{A \vdash_{z} v:: z: U \mid a: \text { use }}{A \vdash_{z} a:=v:: a: \operatorname{rd}(U)}(W r V B) \quad a: \operatorname{rd}(U) ; \text { use } \vdash_{x} a:: x: U \mid a: \text { use }(R d V F) \\
& \frac{A \vdash_{z} v:: z: \circ U \mid a: \mathrm{wr}(\circ U)}{A \vdash_{z} a:=v:: 0}(W r V F) \quad a: \operatorname{rd}(U) \vdash_{x} a:: x: U(R d V B) \frac{A \vdash_{x} e:: x: T}{A \vdash_{x} \text { fork } e:: x: \tau(T)}(\text { Fork }) \frac{A \vdash_{x} e:: x: \tau(T)}{A \vdash_{x} \text { wait } e:: x: T}(\text { Wait })
\end{aligned}
$$

Figure 4. Typing Rules.

Let $U$ be some shared isolated type (e.g. a type such that $U<$ : !o $U$ ). We can then derive the typing

$$
\text { atomic: } U \mapsto(\text { !set: }(U \mapsto 0) \mid \text { !get: } U)
$$

by associating to lock the invariant $s: \operatorname{rd}(U)$; var. This type states that atomic is a function that returns a stateful variable "object" that can be safely used concurrently by an arbitrary number of setters and getters. Notice that if any of the two sync blocks is removed, atomic would only be typed by a behavioral-separation type that sequentializes the get and set operations somehow. For example, if both sync blocks are removed, a possible typing is

$$
\text { atomic: } U \mapsto(\text { !get: } U \text {; set: }(U \mapsto 0))^{*}
$$

which would still allow sharing (aliasing, or concurrent usage) of the get "method" but not of the set "method".

This example illustrates how the monitor construction can be explained as a type coercion operation in our type structure, e.g., coercing $A ; B$ to $A \mid B$, or even $(A ; B) \&(B ; A)$ to $A \mid B$. Recall that by the exchange law we can derive $A \mid B<:(A ; B) \&(B ; A)$, expressing the basic interleaving principle that a value of type $A \mid B$ can be used according to $(A ; B) \&(B ; A)$. Conversely, given a value providing the behavior $(A ; B) \&(B ; A)$, we may in general coerce it to the behavior $A \mid B$, by wrapping within a monitor that enforces the appropriate usage protocol by means of locking. The monitored object then exports two behaviorally parallel separated interfaces $A$ and $B$, even if there is potentially sharing / interference between the implementations of $A$ and $B$. Our type system naturally assigns $A \mid B$ to the monitored object, relying on the modular type rules for locking and on standard invariant-based reasoning.

Invariant based reasoning is also useful in a non-concurrent setting, in which case we may consider the sync operator essentially as a typing device for potentially shared (aliased) usages. We elaborate on this point using a simple, yet non-trivial example: a FIFO queue implemented with a linked list data structure with head and tail pointers (code is presented in Figure 5). We describe the type Node assigned to node value in the list. Let

$$
\begin{array}{ll}
\text { Node } \triangleq \text { HeadT } \mid \text { TailT } & \text { SHeadT } \triangleq \operatorname{Opt}(\text { HeadT }) \\
\text { HeadT } \triangleq \text { ounlink: }: \text { SHeadT } & \text { TailT } \triangleq \text { olink: }: \text { SHeadT } \mapsto 0
\end{array}
$$

Our type system assigns to function new the type $(0 \mapsto$ Node $)$.

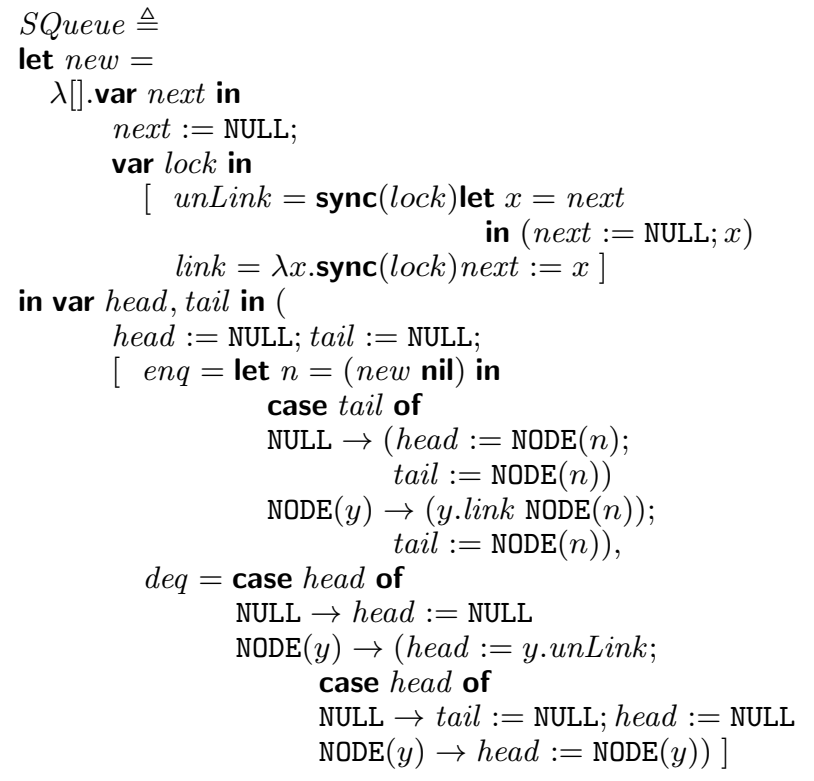

Figure 5. A FIFO Queue Implemented with a Linked List

Node is a parallel separation type, exposing, on the one hand, the behavior to be assigned to the head pointer or to the previous node in the list, and, on the other hand, the behavior to be assigned to the tail pointer. The safe separation of behaviors is here enforced by the use of invariant based separation, associating to lock the invariant next:rd(oSHeadT) ; var.

We can then derive $\vdash_{q}$ SQueue :: SQueueI where

$$
\text { SQueueI } \triangleq(q: \text { enq:0 \& q:deq:0)* }
$$

This type says that the declared behavioral separation protocols are enforced, even in the presence of possible interference between the state accessible from head and tail. Additionally, type Node clearly says that both link and unlink operation are used exactly once in each list node, the first through the tail alias, the other by the head alias, and that this describes the full behavior of a node. 
Type SQueueI above declares a sequential behavior for the queue, where $e n q$ and $d e q$ operations cannot be selected concurrently. A simple typable concurrent queue can be defined by guarding the sequential implementation described with appropriate sync blocks:

$$
\begin{aligned}
& \text { let } C Q \text { ueue }=\text { var head, } \text { tail in }( \\
& \text { head }:=\mathrm{NULL} ; \text { tail }:=\mathrm{NULL} ; \\
& \text { var qinv in } \\
& {\left[\begin{array}{c}
e n q=\operatorname{sync}(q i n v)(\ldots) \\
d e q=\operatorname{sync}(\text { qinv })(\ldots)
\end{array}\right]}
\end{aligned}
$$

For type checking this code we associate to qinv the invariant head:rd(oSHeadT); var|tail:rd(oSTailT); var. We then derive $\vdash_{q} C Q u e u e::$ QueueCI for QueueCI $\triangleq$ !enq:0|!q:deq:0. The $Q u e u e C I$ interface type explicitly says that the queue can be safely used by many concurrent clients, as in. e.g.,

$$
\text { let } q=C Q u e u e \text { in (q.enq; q.enq\|q.deq; q.deq) }
$$

Notice that in the assertion typing SQueue only sequential types appear, that is, no $(-\mid-)$ or $!(-)$. So only a single thread may be visiting the code of SQueue. This means (informal claim) that the lock lock associated to each list node will always be free. So, the sync blocks in Node are operationally irrelevant, and may be seen as an auxiliary device for bracketing code regions subject to invariant-based type checking of separation. On the other hand, sync blocks are essential to CQueue, if it is to be actually used according to the more permissive type QueueCI.

As a further example, we present a code fragment tying a Landin's knot, thus creating a circular chain of references in the higher-order store. We may verify that it can be typed by assigning to the (operationally useless) lock linv the invariant $a: \operatorname{rd}(! \circ(0 \mapsto 0))$; var, and to the function $f$ the type !o $(0 \mapsto 0)$.

$$
\begin{aligned}
& \text { var } a \text { in }(a:=\lambda x . x ; \\
& \quad \text { var } \operatorname{linv} \text { in let } f=\lambda y .(\operatorname{sync}(\operatorname{linv})(a) y) \\
& \quad \text { in }(\operatorname{sync}(\text { linv })(a:=f) ;(f \text { nil })))
\end{aligned}
$$

In principle, our type system could be refined to distinguish between two different scenarios for invariant based reasoning, one already useful to handle interference in sequential code, another one to handle interference in truly concurrent code, only the latter would require real locks to be introduced in the code. We leave this discussion for future consideration, for the issue seems orthogonal to the main purpose of this paper. A key point to highlight here is that our typing principles for the sync construct seem to capture a useful and general form of invariant reasoning about safe interference in the context of a behavioral separation type system.

We can now state the type preservation result for the full core language. To that end, typing for runtime configurations is generalized to consider the declaration of invariants. This is achieved by a global invariant mapping $\iota$, which assigns lock invariants to locations: essentially, the rule $(\mathrm{H})$ of Section 4.4 is replaced by the following rules, covering the two possible lock states (see Appendix).

$$
\begin{gathered}
\frac{h ; S \triangleright_{\iota} A \mid R \quad A \vdash_{x}^{\iota} v:: B}{h, n_{0} \mapsto v ; S \triangleright_{\llcorner\{R / n\}} B\{x / n: \mathrm{var}\}} \\
\frac{h ; S \triangleright_{\iota} A \quad A \vdash_{x}^{\iota} v:: B}{h, n_{-1} \mapsto v ; S \triangleright_{\llcorner\{R / n\}} B\{x / n: \operatorname{var}\}}
\end{gathered}
$$

Theorem 4.5. If $h ; S \triangleright_{\iota} A$ and $h ; S \rightarrow h^{\prime} ; S^{\prime}$ then $h^{\prime} ; S^{\prime} \triangleright_{\iota} A$.

A progress property also holds for the full core language with concurrency and synchronization primitives, but in a restricted sense, due to the possibility of deadlock on sync blocks (see Appendix).

\section{Related Work}

The concept of separation results from a research stream whose origins can be traced back to the seminal works of Reynolds on syntactic control of interference [32, 34]. Separation logics extend classical Hoare logic with new connectives, in particular the separation conjunction, which allows to specify the fine-grained structure of states in programs manipulating references, and enables local reasoning to successfully tackle programs with references [33] and concurrency [30]. More recently, separation logic has motivated the introduction of Hoare Type Theory [28], and has been extended to languages with higher-order store [36]. These works focus on the identification of higher-order frame principles for state-based local reasoning. The idea of assigning a parallel separation type to some value, even when there is (safe) interference between the implementations of the separated behaviors, is reminiscent of concepts explored in fictional separation [23] and concurrent abstract predicates [15]. In our case, we consider such usage behaviors as truly separate, as they can be safely used by independent clients. The focus of behavioral separation is on externally usable protocols of program entities, rather than on the internal state of programs. Although it is clear that the fundamental notion of separation applies to many kinds of computational structures $[11,13,18]$, the idea of combining separation with behavioral types to discipline interference in a realistic programming language, as we do here, does not seem to have been considered before.

Various forms of behavioral types has been independently introduced by several authors with the intent of classifying usage patterns of computational objects $[19,14,22]$. Some of these works have motivated more refined verification techniques, for example, to check resource usage disciplines [21] in functional programs. A particular case of behavioral types are the so-called session types, intended to discipline message exchanges between partners in distributed systems. Although initially proposed for systems with interaction between exactly two partners [19], session types have been extended to systems with an arbitrary number of participants [20]. Our notion of type assertion is loosely related with the notion of global type introduced in [20], in the sense that it needs to talk about the joint behavior of several entities. A version of session types to discipline interactions between concurrent objects was developed in [16], but does not attempt to deal with interference or aliasing. More recently, we have developed an interpretation of session types in linear logic [12], which also inspired some aspects of the theory presented here. Connections between session types and separation logic have also been investigated in [37], but focusing on disciplining the transference of resources in process communications. In prior work, we attempted a very preliminary approach to the concept of behavioral separation [10]. However, the developments in this paper clarify the notion of behavioral separation type in the context of a clean substructural type theory, based on a $\lambda$ calculus with imperative and concurrency constructs, and are much more general and expressive.

Several works have proposed type-based approaches to discipline aliasing and concurrency control in various programming languages, usually exploiting type and effect systems $[1,8]$. Ownership types have also been studied to discipline aliasing and concurrency $[7,6]$. Some of these works have led to the development of powerful programming tools [25]. Typically, these works do not focus on capturing the dynamic behavior resources at a deeper level, as we attempt in our work, but on tracking occurrences of identifiers, locks, permissions, regions, and data dependencies, usually resorting to linearity. An important exception is typestate, which uses a state-based approach to specify resource usage protocols in object oriented languages [3]. A key ingredient of the typestate approach is the use of primitive permissions to capture usage idioms, rather than resource behavior. In parallel research we are investigating combinations of separation with typestate [26]. Techniques to support expressive borrowing idioms in the context of typestate have been recently proposed in [27]. 


\section{Conclusions}

We have introduced behavioral separation as a general principle for disciplining interference, either due to aliasing or concurrency, by combining concepts from separation logic and behavioral type systems. We have designed a behavioral separation type system that illustrates the concept using a higher-order imperative functional language extended with concurrency and synchronization primitives. Our type system is proven sound using proof theoretic techniques.

We have also shown that the expressiveness of our approach goes beyond the state of the art for type-based verification of aliasing and concurrency, and provided several challenging examples involving fine-grained state manipulation, thread based concurrency, and synchronization constructs. Further examples, including the implementation of a concurrent queue based on a double linked list can be found in the Appendix. In ongoing work, we are studying algorithmic aspects of our type system; in fact, we have already designed an algorithm that can effectively typecheck programs in our core language with a reasonable annotation burden, these results will be reported elsewhere. We are also investigating generalizations to invariant-based separation, along the lines suggested in Section 4.5.

\section{References}

[1] M. Abadi, C. Flanagan, and S. N. Freund. Types for safe locking: Static race detection for Java. ACM Trans. Program. Lang. Syst., 28(2):207-255, 2006.

[2] A. Ahmed, M. Fluet, and G. Morrisett. L ${ }^{3}$ : A Linear Language with Locations. Fundam. Inform., 77(4):397-449, 2007.

[3] K. Bierhoff and J. Aldrich. Modular Typestate Checking of Aliased Objects. In OOPSLA 2007, pages 301-320, 2007.

[4] S. L. Bloom and Z. Ésik. Free Shuffle Algebras in Language Varieties. Theoretical Computer Science, 163(1\&2):55-98, 1996.

[5] R. Bornat, C. Calcagno, P. W. O'Hearn, and M. J. Parkinson. Permission accounting in separation logic. In POPL 2005, pages 259-270, 2005.

[6] C. Boyapati, R. Lee, and M. C. Rinard. Ownership types for safe programming: preventing data races and deadlocks. In OOPSLA 2002, pages 211-230. ACM, 2002.

[7] C. Boyapati, B. Liskov, and L. Shrira. Ownership types for object encapsulation. In POPL 2003, 2003.

[8] C. Boyapati and M. C. Rinard. A Parameterized Type System for Race-Free Java Programs. In OOPSLA 2001, pages 56-69, 2001.

[9] J. Boyland. Checking Interference with Fractional Permissions. In SAS 2003, volume 2694 of Lecture Notes in Computer Science, pages 55-72. Springer-Verlag, 2003.

[10] L. Caires. Spatial-Behavioral Types for Concurrency and Resource Control in Distributed Systems. Theoretical Computer Science, 402(2-3):120-141, 2008.

[11] L. Caires and L. Cardelli. A Spatial Logic for Concurrency (Part I). Information and Computation, 186(2):194-235, 2003.

[12] L. Caires and F. Pfenning. Session Types as Intuitionistic Linear Propositions. In CONCUR'10, volume 6269 of Lecture Notes in Computer Science, pages 222-236. Springer-Verlag, 2010.

[13] C. Calcagno, P. W. O'Hearn, and H. Yang. Local Action and Abstract Separation Logic. In 22nd IEEE Symposium on Logic in Computer Science (LICS 2007), pages 366-378, 2007.

[14] S. Chaki, S. K. Rajamani, and J. Rehof. Types as models: Model Checking Message-Passing Programs. In POPL 2002, pages 45-57, 2002.

[15] T. Dinsdale-Young, M. Dodds, P. Gardner, M. J. Parkinson, and V. Vafeiadis. Concurrent Abstract Predicates. In ECOOP 2010, volume 6183 of Lecture Notes in Computer Science, pages 504-528.
Springer-Verlag, 2010.

[16] S. J. Gay, V. Thudichum Vasconcelos, A. Ravara, N. Gesbert, and A. Z. Caldeira. Modular Session Types for Distributed Objectoriented Programming. In POPL 2010, pages 299-312, 2010.

[17] C. A. R. Hoare, B. Möller, G. Struth, and I. Wehrman. Concurrent Kleene Algebra. In M. Bravetti and G. Zavattaro, editors, CONCUR'09, volume 5710 of Lecture Notes in Computer Science, pages 399-414. Springer-Verlag, 2009.

[18] T. Hoare and P. W. O'Hearn. Separation Logic Semantics for Communicating Processes. ENTCS, 212:3-25, 2008.

[19] K. Honda, V. T. Vasconcelos, and M. Kubo. Language Primitives and Type Discipline for Structured Communication-Based Programming. In ESOP 1998, volume 1381 of Lecture Notes in Computer Science, pages 122-138. Springer-Verlag, 1998.

[20] K. Honda, N. Yoshida, and M. Carbone. Multiparty Asynchronous Session Types. In POPL 2008, pages 273-284. ACM, 2008.

[21] A. Igarashi and N. Kobayashi. Resource Usage Analysis. In POPL 2002, pages 331-342, 2002.

[22] A. Igarashi and N. Kobayashi. A Generic Type System for the PiCalculus. Theoretical Computer Science, 311(1-3):121-163, 2004.

[23] J. B. Jensen and L. Birkedal. Fictional Separation Logic. In ESOP 2012, Lecture Notes in Computer Science, pages 377-396. SpringerVerlag, 2012.

[24] Cliff B. Jones. Splitting Atoms Safely. Theor. Comput. Sci., 375(13):109-119, 2007.

[25] R. L. Bocchino Jr., S. Heumann, N. Honarmand, S. V. Adve, V. S. Adve, A. Welc, and T. Shpeisman. Safe Nondeterminism in a Deterministic-by-default Parallel Language. In POPL 2011, pages 535-548, 2011.

[26] Militão, F. and Aldrich, J. and Caires, L. Aliasing control with view-based typestate. In FTFJP '10, pages 7:1-7:7. ACM, 2010.

[27] K. Naden, R. Bocchino, J. Aldrich, and K. Bierhoff. A Type System for Borrowing Permissions. In POPL 2012, pages 557-570, 2012.

[28] A. Nanevski, J. G. Morrisett, and L. Birkedal. Hoare Type Theory, Polymorphism and Separation. J. Fun. Prog., 18(5-6):865-911, 2008.

[29] Peter W. O'Hearn. On Bunched Typing. J. Fun. Prog., 13(4):747796, 2003.

[30] Peter W. O'Hearn. Resources, Concurrency, and Local Reasoning. Theor. Comput. Sci., 375(1-3):271-307, 2007.

[31] B. Pierce. Types and Programming Languages. MIT Press, 2002.

[32] J. C. Reynolds. Syntactic Control of Interference. In POPL 78, pages 39-46, 1978.

[33] J. C. Reynolds. Separation Logic: A Logic for Shared Mutable Data Structures. In Third Annual Symposium on Logic in Computer Science, Copenhagen, Denmark, 2002. IEEE Computer Society.

[34] John C. Reynolds. Syntactic Control of Interference, Part 2. In G. Ausiello, M. Dezani-Ciancaglini, and S. Ronchi Della Rocca, editors, ICALP 89, volume 372 of Lecture Notes in Computer Science, pages 704-722. Springer-Verlag, 1989.

[35] John C. Reynolds. Design of the programming language FORSYTHE, pages 173-233. Birkhauser Boston Inc., Cambridge, MA, USA, 1997.

[36] J. Schwinghammer, L. Birkedal, B. Reus, and H. Yang. Nested Hoare Triples and Frame Rules for Higher-order Store. Logical Methods in Computer Science, 7(3), 2011.

[37] J. Villard, E. Lozes, and C. Calcagno. Proving copyless message passing. In Programming Languages and Systems, 7th Asian Symposium, APLAS 2009, volume 5904 of Lecture Notes in Computer Science, pages 194-209. Springer-Verlag, 2009. 


\section{A. Appendix}

\section{A.1 Recursion}

To accommodate recursion (in both programs and types) in the type system, we add to typing judgements a recursion variable environment $\eta$, which maps expression variables $Z$ to type judgments and type variables $X$ to type assertions. This technique allows recursion to be interpreted coinductively; our use of a recursion environment is inspired in familiar techniques (e.g., [31]). We include two pair of rules, one pair for recursive expression and expression variable, and other pair for recursive type and type variable.

$$
\begin{array}{cc}
\left.\frac{\eta(X)=A}{A \vdash_{x}^{\eta} v:: X} \text { (VRecVar }\right) & \frac{A \vdash_{x}^{\eta\{X / A\}} v:: B}{A \vdash_{x}^{\eta} v:: \operatorname{rec}(X) B}(\text { VRec }) \\
\frac{\eta(Z)=\left(A \vdash_{x} B\right)}{A \vdash_{x}^{\eta} Z:: B}(\text { RecVar }) & \frac{A \vdash_{x}^{\eta\left\{Z /\left(A \vdash_{x} B\right)\right\}} e:: B}{A \vdash_{x}^{\eta} \operatorname{rec}(Z) e:: B}(\operatorname{Rec})
\end{array}
$$

We have elided the recursion environment in typing judgments in most of our presentation in the paper main body. For the sake of clarity, we have also stated our technical results for the recursion free language and type system, since recursion is treated along standard lines, and fairly orthogonal to the new aspects of our development. Both the recursion environment $\eta$ and the invariant mapping $\iota$ should be propagated without modification from premises to conclusion of all typing rules in Figure 4. Nevertheless, for completeness of our presentation, we collect in Figure 8 the full set of rules of our type system, based on the complete judgment form

$$
A \vdash_{z}^{\iota, \eta} e:: B \quad \text { ( } e \text { types from } A \text { to } z \text { in } B \text { under } \iota, \eta \text { ) }
$$

\section{A.2 Runtime Configuration Typing}

We summarize the runtime configuration typing rules for the full language with synchronization primitives.

$$
\begin{gathered}
\emptyset ; \emptyset \triangleright_{\emptyset} 0 \text { (E) } \\
\frac{h ; S \triangleright_{\iota} \mathcal{E}[C] \quad C \vdash_{x}^{\iota, \emptyset} e:: x: T}{h ; S \cdot t\langle e\rangle \triangleright_{\iota} \mathcal{E}[t: \tau(T)]} \\
\frac{h ; S \triangleright_{\iota} A \mid R \quad A \vdash_{x}^{\iota, \emptyset} v:: B}{h, n_{0} \mapsto v ; S \triangleright_{\iota\{R / n\}} B\{x / n: \operatorname{var}\}} \\
\frac{h ; S \triangleright_{\iota} A \quad A \vdash_{x}^{\iota, \emptyset} v:: B}{h, n_{-1} \mapsto v ; S \triangleright_{\iota\{R / n\}} B\{x / n: \operatorname{var}\}} \\
\frac{h ; S \triangleright_{\iota} A \quad A<: B}{h ; S \triangleright_{\iota} B} \quad \text { (S) }
\end{gathered}
$$

N.B. In $(H U), R$ is a lock invariant (see (Section 4.5). The notation $B\{x / n$ :var $\}$ (with $n$ fresh in $B$ ) represents the transformation of type $B$ where the behavior at $x \in \operatorname{Dom}(B)$ becomes provided through a new heap cell $n$. We formally define $A\{x / n$ :var $\}$ using the auxiliary substitution form $A[x / n$ :var $]$ :

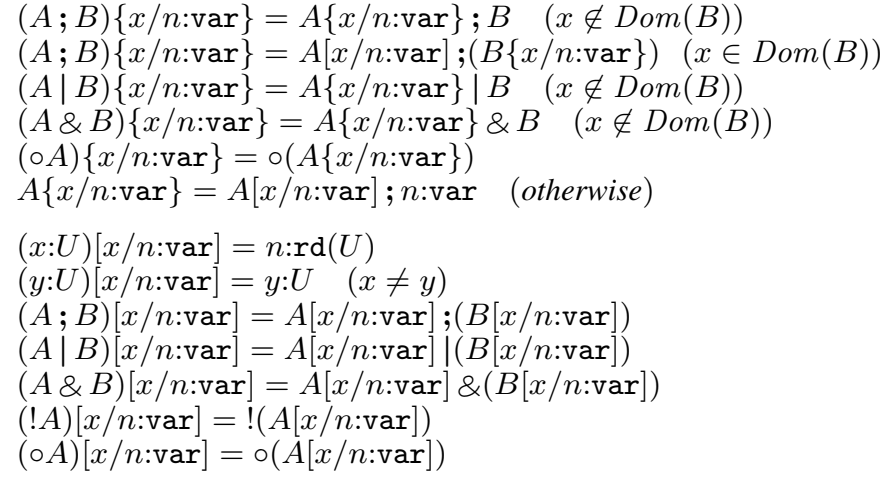

(We omit the symmetric cases of $\mid$ and \&). Notice that $B\{x / n$ :var $\}$ preserves the standard variable protocol for $n$, and correctly inserts the terminal behavior $n$ :var at the earliest possible position after all the $n: \operatorname{rd}(-)$ declarations. If $B=(x: U \mid z: T) ; a: V$ with $x \neq z$ then $B\{x / n: \operatorname{var}\}=(n:(\operatorname{rd}(U) ; \operatorname{var}) \mid z: T) ;(a: V)$, but if $B=$ $(x: U \mid x: T) ; a: V$ then $B\{x / n: \operatorname{var}\}=n:(\operatorname{rd}(U \mid T) ; \operatorname{var}) ; a: V$.

It should be clear that if $h ; S \triangleright A$ then the type $A$ only talks about the usage of heap variables in $h$ and threads in $S$.

\section{A.3 Further Remarks on Type Safety}

By Theorems 4.3, 4.4, and 4.5, well typed programs satisfy all the declared behavioral separation protocols, and the progress property holds even in the presence of strong updates (changing types) of heap variables. We expand on the remark in Section 4.4:

Proposition A.1 (Race absence). Runtime configurations of the following forms cannot be typed.

1. (write/read race) $h ; S \cdot t_{1}\left\langle\mathcal{F}_{1}[n:=u]\right\rangle \cdot t_{2}\left\langle\mathcal{F}_{2}[n]\right\rangle \triangleright_{\iota} A$.

2. (write/write race) $h ; S \cdot t_{1}\left\langle\mathcal{F}_{1}[n:=u]\right\rangle \cdot t_{2}\left\langle\mathcal{F}_{2}[n:=v]\right\rangle \triangleright_{\iota} A$.

Proof. By induction on typing derivations, using the key fact that for the post-condition type $B$ of a well-typed configuration typing $h ; S \triangleright_{\iota} B$ we cannot have $B<:(n: \operatorname{rd}(V) \mid n: \operatorname{wr}(U)) ; B^{\prime}$ or $B<:(n: \operatorname{wr}(V) \mid n: \operatorname{wr}(U)) ; B^{\prime}$, given the subtyping axiomatization of var (Section 4.1), and runtime configuration typing.

\section{A.4 A double linked List with Concurrent Iterator}

We discuss types for the implementation of a FIFO queue over a double linked list, thus refining the development in Section 4.5. This example complements the discussion in Section 4.2.8, and illustrates how behavioral separation and the typing constraints on heap allocated variables do not forbid all circular chains of references in the heap. It also demonstrates how behavioral separation types precisely capture the safe usage discipline of the queue iterator "method", which in our example supports concurrent transversals of the linked list. The code is shown in Figure 6 . Consider first the code for the list nodes, generated by the function DNode, and which expose operations setNext, getNext, setPrev, getPrev. We propose some type declarations that may be used to check our implementation. These are not the most expressive ones one might consider for the code at hand, but sufficient to make several interesting points.

$$
\begin{array}{ll}
G \triangleq \operatorname{getNext}: ! O N & O N \triangleq \operatorname{Opt}(G) \\
O H \triangleq \operatorname{pt}(\circ H) & H \triangleq(G ; \circ H) \&(P ; ! \circ G) \\
O T \triangleq \operatorname{pt}(\circ T) & P \triangleq \operatorname{setPrev}:(\circ O T \mapsto 0) \\
T \triangleq \operatorname{getPrev}: \circ O T & N \triangleq \circ T \mid(\operatorname{setNext}:(! \circ O N \mapsto 0) ; \circ H)
\end{array}
$$

We can derive $0 \vdash_{z} D N o d e:: z: 0 \mapsto N$, where $N$ is the behavioral separation type of a single list node. To typecheck the synchronized blocks in operations getPrev and setPrev the lock invariant assertion associated to $i n v$ is $\mathrm{rd}(\circ O T)$; var.

The queue provides three operations: enq (enqueue an element), deq (dequeue the oldest element, if any), and iter. The operation iter returns an iterator "object", which can be used concurrently by an arbitrary number of clients (each one invoking a scan operation), before being released (by means of a done operation). The iterator object is assigned the following interface type

$$
I T \triangleq \operatorname{SOME}(! \text { scan }: 0 ; \text { done }: 0) \oplus \text { NONE }
$$

The option NONE signals that the queue is empty, $\operatorname{SOME}(-)$ that there are indeed some elements to scan.

We set $Q T \triangleq(e n q: 0 \text { \& deq:0 \& iter:IT })^{*}$ as the behavioral separation type describing the safe usage protocol of the queue code. We can derive $0 \vdash_{q}$ DList :: $q: Q T$. 
DNode $\triangleq$

$$
\begin{aligned}
& \lambda[] \text {.var next, prev in } \\
& \text { prev }:=\text { NULL; } \\
& \text { var } i n v \text { in } \\
& \text { [ } \text { getNext }=\text { next } \\
& \text { setNext }=\lambda x .(\text { next }:=x) \\
& \text { getPrev }=\operatorname{sync}(\text { inv }) \text { let } x=\text { prev } \\
& \text { in }(\text { prev }:=\text { NULL; } x) \\
& \text { setPrev }=\lambda x \cdot \operatorname{sync}(\text { inv })(\text { prev }:=x)]
\end{aligned}
$$

DList $\triangleq$

var head, tail in (

head $:=$ NULL; tail $:=$ NULL;

[ enq $=$ let $n=($ DNode nil $)$ in

case head of

NULL $\rightarrow((n . s e t N e x t$ NULL $)$;

head $:=\operatorname{NODE}(n)$;

tail $:=\operatorname{NODE}(n))$

$\operatorname{NODE}(y) \rightarrow((y . s e t P r e v \operatorname{NODE}(n))$

(n.setNext $\operatorname{NODE}(y))$;

$d e q=$ case tail of

head $:=\operatorname{NODE}(n))$,

NULL $\rightarrow$ tail $:=$ NULL

$\operatorname{NODE}(y) \rightarrow($ tail $:=y . g e t P r e v ;$

case tail of

NULL $\rightarrow$ tail $:=$ NULL; head $:=$ NULL

iter $=$ case head of

$\operatorname{NODE}(z) \rightarrow$ tail $:=\operatorname{NODE}(z))$

NULL $\rightarrow$ head $:=$ NULL; NONE

$\operatorname{NODE}(n) \rightarrow$ head $:=\mathbf{n i l}$;

let $s=n . g e t N e x t$ in

let $i o=[$

scan $=$

var $a$ in $(a:=s$;

$\operatorname{rec}(L)$.case $a$ of

NULL $\rightarrow$ nil

$\operatorname{NODE}(r) \rightarrow$

$(a:=r . g e t N e x t ; L))$,

done $=($ head $:=\operatorname{NODE}(n)]$

in $\operatorname{SOME}(i o)$

Figure 6. Queue over a double linked list, and iterator

After the two initial assignments (of NULL) to head and tail, these heap variables keep as invariant (between "method" invocations) the types $\operatorname{rd}(\circ O H)$; var and $\operatorname{rd}(\circ O T)$; var, respectively.

Consider the client code snippet $P_{1}$ in Figure 7 . We have $q: Q T \vdash_{x} P_{1}:: 0$, so $0 \vdash_{x}$ let $q=$ DList in $P_{1}:: 0$.

Thus the composition of the queue and client code typechecks, and thus free from protocol violations, including races on heap variable access. On the other hand, consider the code snippet $P_{2}$ : it enqueues an element in the queue after requesting an iterator ( $i=$ q.iter , but before using the iterator $i$. Due to aliasing, the s.done operation may erroneously interfere with the mentioned enqueue operation, thus breaking the invariant that head should always refer to the first element and tail to the last element of the queue. Defensively, we have safeguarded against such misusage by setting head to nil at iterator construction time (line head := nil ).

So, in fact, $P_{2}$ "crashes" during evaluation of the last q.enq expression, since the code for enq expects head to hold a value of sum type $\circ O H$ (such a "crash" is signaled in our semantics by the fact that the case head of ... expression will get stuck).

$$
P_{1} \triangleq
$$$$
\text { q.enq; q.enq; q.deq; }
$$$$
\text { let } i=q . i t e r \text { in }(
$$

$P_{2} \triangleq$

q.enq; q.enq; q.deq;

let $i=q . i t e r$ in (

q.enq;

case $i$ of

NONE $\rightarrow$ nil

$\operatorname{SOME}(s) \rightarrow$

let $f=$ fork $($ s.scan $)$ in

(s.scan; wait $(f) ;$ s.done $)$ )

Figure 7. Some Client Code

Obviously, $P_{2}$ does not typecheck under the assumption $q: Q T$ ! The pre-condition of the body of expression (let $i=q$.iter in $\cdots)$ is $(i: I T ; q: Q T)$, thus forcing the behavior $i: I T$ to be exercised before $q: Q T$ (sequential separation). Likewise, in $P_{1}$ the type precondition of expression (let $f$ in $\cdots$ ) right after $\operatorname{SOME}(s) \rightarrow$ is

$$
(s: ! \text { scan }: 0 ; \text { s:done }: 0 ; q: Q T)
$$

thus allowing multiple threads to perform scan concurrently (parallel separation), but forcing $s$ :done to be executed (exactly once) before proceeding with further queue operations, as shown.

Notice that the concurrent usages of the scan operation, expressed by the type !scan:0 do not require locks in the implementation, since they only rely on read capabilities of shared resources (the cursor variable $a$ is typed $a: ! O N$ ). On the other hand, the synchronized blocks in DNode have been introduced to allow invariant-based reasoning justify the safe sharing of list nodes by head and tail, and do not play an essential role in operational terms, as discussed in Section 4.5.

\section{A.5 Proofs of Main Results}

We present proofs for the main (and some auxiliary) results in the paper. For clarity, we prove type preservation and progress for the basic type system, and afterwards address the full system with synchronization primitives.

We start by stating a few basic inversion principles for the typing relation (which only apply to values $v$ ).

LEMMA A.2 (Inversions). We have

1. If $A \vdash_{x} v:: B$ and $x \notin D o m(B)$ then $A<: B$ and $B \vdash_{x} v:: B$.

2. If $A \vdash_{x} v:: B_{1} \mid B_{2}$ then there are $A_{1}, A_{2}$ such that $A<: A_{1} \mid A_{2}$ and $A_{1} \vdash v:: B_{1}$ and $A_{2} \vdash v:: B_{2}$.

3. If $A \vdash_{x} v:: B_{1} ; B_{2}$ then there are $A_{1}, A_{2}$ such that $A<: A_{1} ; A_{2}$ and $A_{1} \vdash v:: B_{1}$ and $A_{2} \vdash v:: B_{2}$.

4. If $A \vdash_{x} v:: ! B$ then there are $A_{i}$ such that $A<: ! A_{1}|\cdots| ! A_{n}$ and $! A_{1}|\cdots| ! A_{n} \vdash v:: B$.

5. If $A \vdash_{x} v:: \circ B$ then there are $A_{i}$ such that $A<: \circ A_{1}|\cdots| \circ A_{n}$ and $\circ A_{1}|\cdots| \circ A_{n} \vdash v:: B$.

Proof. By induction on typing derivations.

A generalized substitution lemma holds for the type system, which corresponds essentially to a natural cut principle in the behavioral separation type structure. 
Lemma A.3. If $A \vdash_{x} v:: B$ and $B \vdash_{z} e:: C$ then there is $D$ such that $A \vdash_{z} e\{v / x\}:: D$ and $D \vdash_{x} v:: C$.

Proof. By induction on the derivation of $B \vdash_{z} e:: C$. Notice that $x$ may occur in $B$, but not in $A$. In the statement conclusion, the judgment $D \vdash_{x} v:: C$ expresses the usage of $v$ that remains after the use of $x$ actually performed by $e$; this stronger property is particularly useful to handle the let expression case.

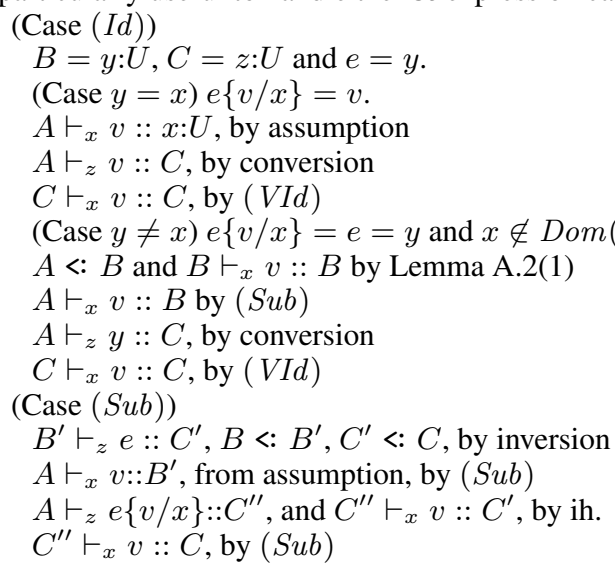

(Case $($ Par $)$ )

$B=B_{1}\left|B_{2}, C=C_{1}\right| B_{2}, B_{1} \vdash_{z}$ e :: $C_{1}$, by inversion

$A_{1} \vdash_{x} v:: B_{1}, A_{2} \vdash_{x} v:: B_{2}$ and $A<: A_{1} \mid A_{2}$

by Lemma A.2(2)

$A_{1} \vdash_{z} e\{v / x\}:: C_{1}^{\prime}$, and $C_{1}^{\prime} \vdash_{x} v:: C_{1}$, by ih.

$A_{1}\left|A_{2} \vdash_{z} e\{v / x\}:: C_{1}^{\prime}\right| A_{2}$, by (Par)

$A \vdash_{z} e\{v / x\}:: C_{1}^{\prime} \mid A_{2}$, by $(S u b)$

$C_{1}^{\prime}\left|A_{2} \vdash_{x} v:: C_{1}\right| A_{2}$, by (Par)

$x \notin B_{2}$ so $A_{2}<$ : $B_{2}$ by Lemma A.2(1)

$C_{1}^{\prime} \mid A_{2} \vdash_{x} v:: C$, by $(S u b)$

(Case $(S e q)$ )

$B=B_{1} ; B_{2}, C=C_{1} ; B_{2}, B_{1} \vdash_{z} e:: C_{1}$, by inversion

$A_{1} \vdash_{x} v:: B_{1}, A_{2} \vdash_{x} v:: B_{2}$ and $A<: A_{1} ; A_{2}$

by Lemma A.2(3)

$A_{1} \vdash_{z} e\{v / x\}:: C_{1}^{\prime}$, and $C_{1}^{\prime} \vdash_{x} v:: C_{1}$, by ih.

$A_{1} ; A_{2} \vdash_{z} e\{v / x\}:: C_{1}^{\prime} ; A_{2}$, by $(S e q)$

$A \vdash_{z} e\{v / x\}:: C_{1}^{\prime} ; A_{2}$, by $(S u b)$

$C_{1}^{\prime} ; A_{2} \vdash_{x} v:: C_{1} ; A_{2}$, by $(S e q)$

$x \notin B_{2}$ so $A_{2}<: B_{2}$ by Lemma A.2(1)

$C_{1}^{\prime} ; A_{2} \vdash_{x} v:: C$, by $(S u b)$

(Case $(V A b s))$

$e=\lambda u . e^{\prime}, C=z: U \mapsto T, B \mid u: U \vdash_{y} e^{\prime}:: y: T$, by inversion

$A\left|u: U \vdash_{x} v:: B\right| u: U$, by (Par)

$A \mid u: U \vdash_{y} e^{\prime}\{v / x\}:: y: T^{\prime}$, and $y: T^{\prime} \vdash_{x} v:: y: T$, by ih.

$x \notin y: T$ so $T^{\prime}<: T$ by Lemma A.2(1)

$A \mid u: U \vdash_{y} e^{\prime}\{v / x\}:: y: T$, by $(S u b)$

$A \vdash_{z} e\{v / x\}:: C$, by $(V A b s)$

$C \vdash_{x} v:: C$, by $(V I d)$

(Case $(A p p))$

$e=e_{1} e_{2}, C=z: T, B=B_{1} \mid B_{2}$,

$B_{1} \vdash_{z} e_{1}:: z: U \mapsto T, B_{2} \vdash_{z} e_{2}:: z: U$, by inversion

$A_{1} \vdash_{x} v:: B_{1}, A_{2} \vdash_{x} v:: B_{2}$ and $A<: A_{1} \mid A_{2}$,

by Lemma A.2(2)

$A_{1} \vdash_{z} e_{1}\{v / x\}:: z: U \mapsto T$, tby ih.

$A_{2} \vdash_{z} e_{2}\{v / x\}:: z: U$, by ih.

$A \vdash_{z} e\{v / x\}:: C$, by $(A p p)$

$C \vdash_{x} v:: C$, by $(V I d)$

(Case (VStop))

$e=v^{\prime}, C=B=0$

$A<: 0$ and $0 \vdash_{x} v:: 0$ by Lemma A.2(1)

$A \vdash_{x} v^{\prime}\{v / x\}:: C$ by $($ VStop) and (Sub)

$C \vdash_{x} v:: C$, by $(V I d)$

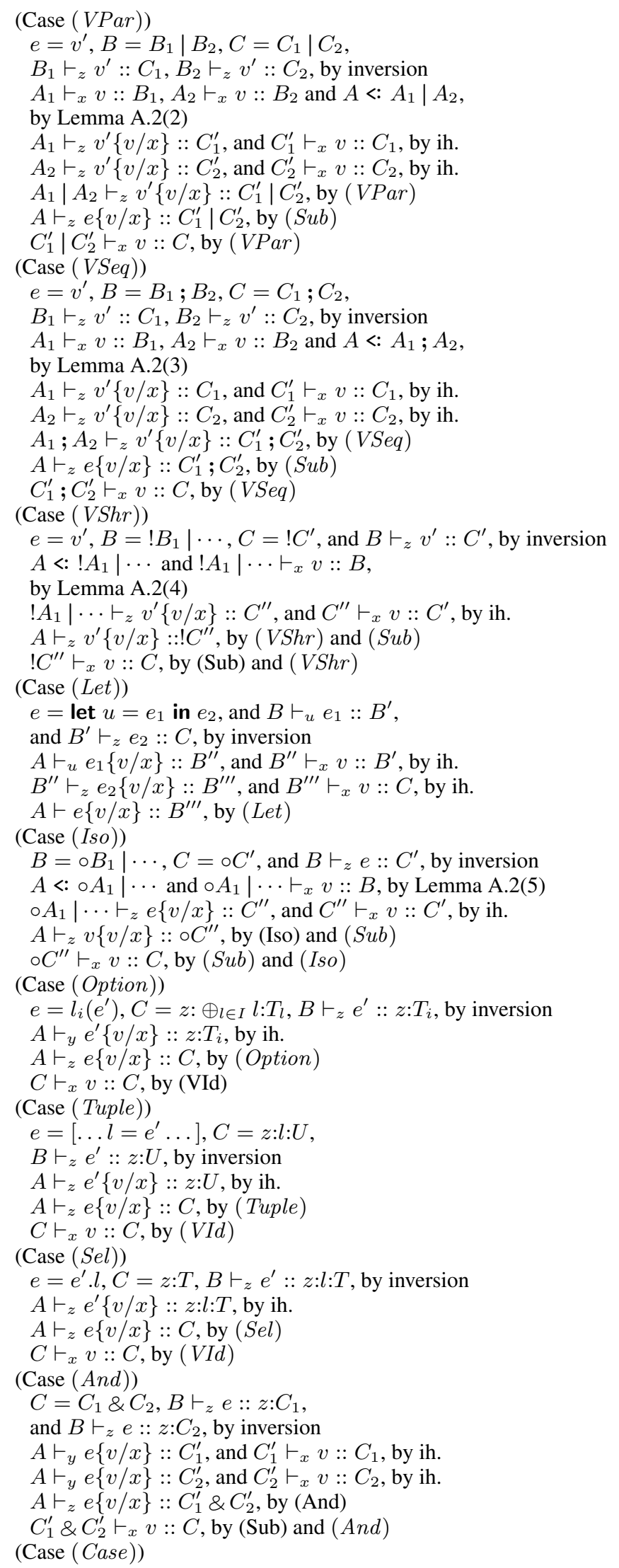

(Case (VPar))

$A_{1} \vdash_{x} v:: B_{1}, A_{2} \vdash_{x} v:: B_{2}$ and $A<: A_{1} \mid A_{2}$,

by Lemma A.2(2)

$A_{1} \vdash_{z} v^{\prime}\{v / x\}:: C_{1}^{\prime}$, and $C_{1}^{\prime} \vdash_{x} v:: C_{1}$, by ih.

$C_{1}^{\prime} \mid C_{2}^{\prime} \vdash_{x} v:: C$, by (VPar)

Case $(V S e q))$

$B_{1} \vdash_{z} v^{\prime}:: C_{1}, B_{2} \vdash_{z} v^{\prime}:: C_{2}$, by inversion

$A_{1} \vdash_{x} v:: B_{1}, A_{2} \vdash_{x} v:: B_{2}$ and $A<: A_{1} ; A_{2}$,

by Lemma A.2(3)

$A_{1} \vdash_{z} v^{\prime}\{v / x\}:: C_{1}$, and $C_{1}^{\prime} \vdash_{x} v:: C_{1}$, by ih

$A_{1} ; A_{2} \vdash_{z} v^{\prime}\{v / x\}:: C_{1}^{\prime} ; C_{2}^{\prime}$, by $(V S e q)$

$A \vdash_{z} e\{v / x\}:: C_{1}^{\prime} ; C_{2}^{\prime}$, by $(S u b)$

$C_{1}^{\prime} ; C_{2}^{\prime} \vdash_{x} v:: C$, by $(V S e q)$

Case $(V S h r))$

$e=v^{\prime}, B=! B_{1} \mid \cdots, C=! C^{\prime}$, and $B \vdash_{z} v^{\prime}:: C^{\prime}$, by inversion

$A \vdash_{z} v^{\prime}\{v / x\}:: ! C^{\prime \prime}$, by (VShr) and (Sub)

$! C^{\prime \prime} \vdash_{x} v:: C$, by (Sub) and $(V S h r)$

$B^{\prime \prime}+e_{1}\{v / x\}: \because B^{\prime \prime}$, and $B^{\prime \prime} F_{x},:: B^{\prime \prime}$, by ih.

$A \vdash e\{v / x\}:: B^{\prime \prime \prime}$, by (Let)

Case $($ Iso $)$

$\circ A_{1} \mid \cdots \vdash_{z} e\{v / x\}:: C^{\prime \prime}$, and $C^{\prime \prime} \vdash_{x} v:: C^{\prime}$, by ih

$A \vdash_{z} v\{v / x\}:: \circ C^{\prime \prime}$, by (Iso) and $(S u b)$

$F_{x} v:: C$, by $(S u b)$ and (Iso)

$A \vdash_{y} e^{\prime}\{v / x\}:: z: T_{i}$, by ih.

$A \vdash_{z} e\{v / x\}:: C$, by (Option)

$C \vdash_{x} v:: C$, by (VId)

$B \vdash_{z} e^{\prime}:: z: U$, by inversion

$C \vdash_{x} v:: C$, by $(V I d)$

$($ Case $(\mathrm{Sel}))$

$e=e^{\prime} . l, C=z: T, B \vdash_{z} e^{\prime}:: z: l: T$, by inversion

$C \vdash_{x} v:: C$, by $(V I d)$

Case $(A n d))$

$C=C_{1} \& C_{2}, B \vdash_{z} e:: z: C_{1}$

$A \vdash_{y} e\{v / x\}:: C_{1}^{\prime}$, and $C_{1}^{\prime} \vdash_{x} v:: C_{1}$, by ih.

政

$C_{1}^{\prime} \& C_{2}^{\prime} \vdash_{x} v:: C$, by (Sub) and (And)

Case (Case)) 


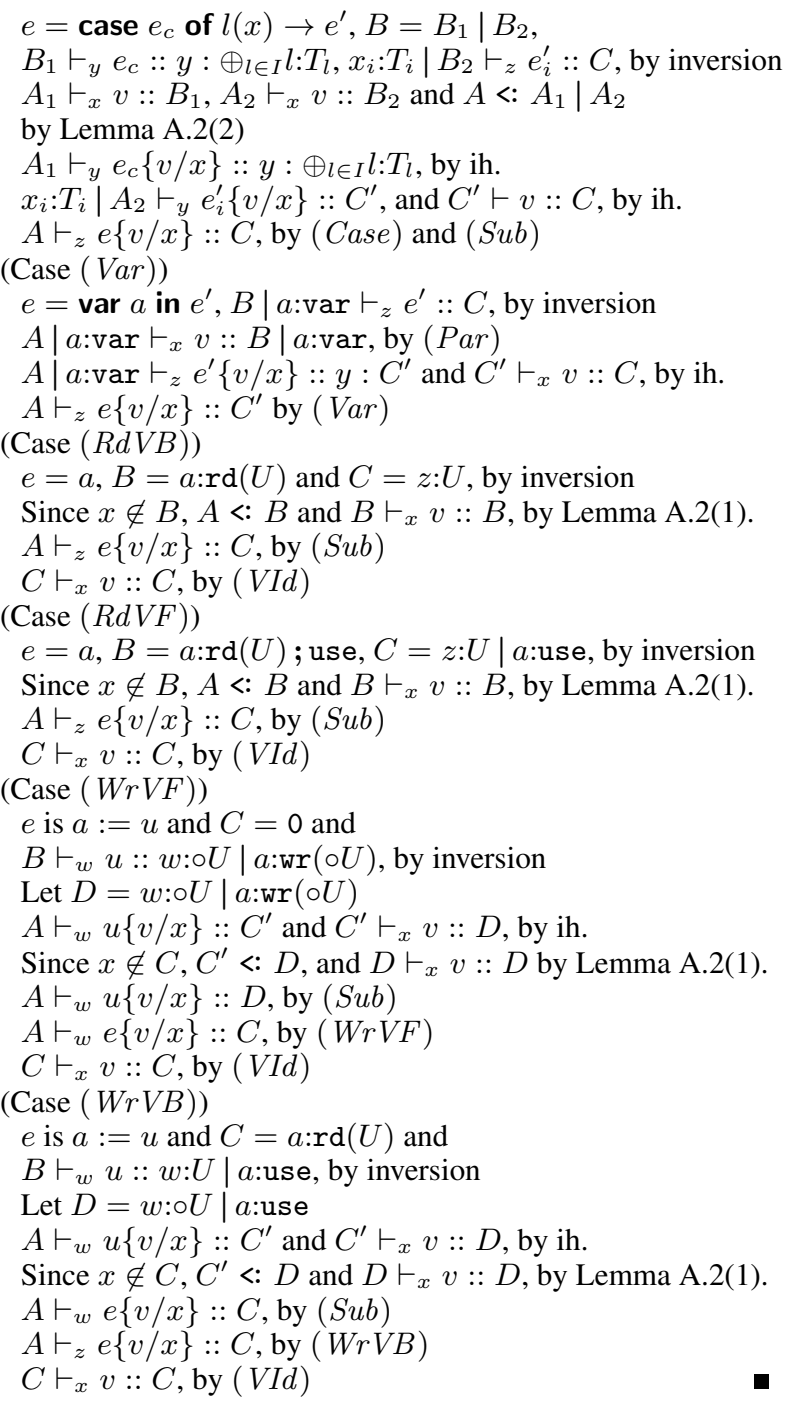

By the previous result, it is straightforward to state and proof the following specialized substitution lemma, useful, for example, to show type preservation of beta reduction.

Lemma A.4. If $A \vdash_{x} v:: x: U$ and $B \mid x: U \vdash_{z} e:: C$ where $x \notin \operatorname{Dom}(C)$ then $B \mid A \vdash_{z} e\{v / x\}:: C$.

Proof. Assume $A \vdash_{x} v:: x: U$.

$B\left|A \vdash_{x} v:: B\right| x: U$, by $($ Par $)$

There is $D$ such that $B \mid A \vdash_{z} e\{v / x\}:: D$

and $D \vdash_{x} v:: C$, by Lemma A.3

$D<: C$ by Lemma A.2(1), hence

$B \mid A \vdash_{z} e\{v / x\}:: C$ by $(S u b)$.

The next lemma covers critical cases of the preservation theorem. We recall that we use a similar notation for evaluation contexts and type assertion contexts $(\mathcal{E}, \mathcal{F})$, without confusion arising.

LEMMA A.5. Let $\mathcal{F}[-]$ be an evaluation context. We have,

1. If $A \vdash_{x} \mathcal{F}[$ let $z=v$ in $e]:: B$ then $A \vdash_{x} \mathcal{F}[e\{v / z\}]:: B$;

2. If $A \vdash_{x} \mathcal{F}[(\lambda z . e) v]:: B$ then $A \vdash_{x} \mathcal{F}[e\{v / z\}]:: B$;

3. If $A \vdash_{x} \mathcal{F}\left[[\overline{l=e}] . l_{i}\right]:: B$ then $A \vdash_{x} \mathcal{F}\left[e_{i}\right]:: B$;

4. If $A \vdash_{x} \mathcal{F}\left[\right.$ case $l_{i}(v)$ of $\left.\overline{l(x) \rightarrow e}\right]:: B$ then

$A \vdash_{x} \mathcal{F}\left[e_{i}\left\{v / x_{i}\right\}\right]:: B$;

5. If $A \vdash_{x} \mathcal{F}[\operatorname{var} a$ in $e]:: B$ then $A \mid n: \operatorname{var} \vdash_{x} \mathcal{F}[e\{n / a\}]:: B$;
6. If $A \vdash_{x} \mathcal{F}[n:=v]:: B$ then there is $C$ such that either

(a) $A<: \mathcal{E}[n$ :use | $C]$ and $\mathcal{E}[n: \operatorname{rd}(U)] \vdash_{x} \mathcal{F}[$ nil $]:: B$ and $C \vdash_{z} v:: z: U$.

(b) $A<: \mathcal{E}[n: \operatorname{wr}(\circ U) \mid C]$ and $\mathcal{E}[0] \vdash_{x} \mathcal{F}[$ nil $]:: B$ and $C \vdash_{z} v:: z: \circ U$.

7. If $A \vdash_{x} \mathcal{F}[n]:: B$ then either

(a) $A$ <: $\mathcal{E}[n: \operatorname{rd}(U)]$ and

for all $C \vdash_{z} v:: z: U$ we have $\mathcal{E}[C] \vdash_{x} \mathcal{F}[v]:: B$;

(b) $A<: \mathcal{E}[n: \operatorname{rd}(U)$; use $]$ and

for all $C \vdash_{z} v:: z: U$ we have $\mathcal{E}[n$ :use $\mid C] \vdash_{x} \mathcal{F}[v]:: B$;

8. If $A \vdash_{x} \mathcal{F}[$ fork $(e)]:: B$ then $A<: \mathcal{E}[C]$ and $C \vdash_{z} e:: z: U$ and $\mathcal{E}[t: \tau(U)] \vdash_{x} \mathcal{F}[t]:: B$, for fresh $t$;

9. If $A \vdash_{x} \mathcal{F}[\operatorname{wait}(t)]:: B$ then $A<: \mathcal{E}[t: \tau(U)]$ and for all $C \vdash_{z} v:: z: U$ we have $\mathcal{E}[C] \vdash_{x} \mathcal{F}[v]:: B$;

Proof. Induction on the typing derivations. We detail some cases of 2 , induction on the derivation of $A \vdash_{x} \mathcal{F}[(\lambda z . e) v]:: B$.

(Case $($ Par $)$ )

$A=A_{1}\left|C, B=B_{1}\right| C$

$A_{1} \vdash_{x} \mathcal{F}[(\lambda z . e) v]:: B_{1}$, by inversion

$A_{1} \vdash_{x} \mathcal{F}[e\{z / v\}]:: B_{1}$, by i.h.

$A \vdash \mathcal{F}[e\{v / z\}]:: B$ by $($ Par $)$

(Case $($ Let))

$\mathcal{F}[-]=$ let $y=\mathcal{G}[-]$ in $e_{2}$

$A \vdash_{y} \mathcal{G}[(\lambda z . e) v]:: C, C \vdash_{x} e_{2}:: B$, by inversion

$A \vdash_{y} \mathcal{G}[e\{z / v\}]:: C$, by i.h.

$A \vdash \mathcal{F}[e\{v / z\}]:: B$ by $($ Let $)$

(Case $(A p p))$

$\mathcal{F}[(\lambda z . e) v]=e_{1} e_{2}, B=x: T, A=A_{1} \mid A_{2}$,

$A_{1} \vdash_{y} e_{1}:: y: U \mapsto T, A_{2} \vdash_{w} e_{2}:: w: U$, by inversion

$($ Case $\mathcal{F}[-]=\square)$

$e_{1}=\lambda z . e, e_{2}=v$

$A_{1} \mid z: U \vdash_{y}$ e :: $y: T$, by inversion

$A_{2} \vdash_{z} v:: z: U$ by $($ Par $)$

$A_{1} \mid A_{2} \vdash_{y} e\{v / z\}:: y: T$ by Lemma A.4

$A \vdash_{x} e\{v / z\}:: x: T$, by renaming

$\left(\right.$ Case $\mathcal{F}[-]=\mathcal{G}[-] e_{2}$, with $\left.\mathcal{G}[(\lambda z . e) v]=e_{1}\right)$

$A_{1} \vdash_{y} \mathcal{G}[(\lambda z . e) v]:: y: U \mapsto T$.

$A_{1} \vdash_{y} \mathcal{G}[e\{v / z\}]:: y: U \mapsto T$, by i.h.

$A \vdash \mathcal{G}[e\{v / z\}] e_{2}:: B, A \vdash \mathcal{F}[e\{v / z\}]:: B$ by $(A p p)$

$\left(\right.$ Case $\mathcal{F}[-]=v_{1} \mathcal{G}[-]$, with $e_{1}=v_{1}$, and $\left.\mathcal{G}[(\lambda z . e) v]=e_{2}\right)$

$A_{2} \vdash_{w} \mathcal{G}[(\lambda z . e) v]::: w: U$

$A_{2} \vdash{ }_{w} \mathcal{G}[e\{v / z\}]:: w: U$, by i.h.

$A \vdash e_{1} \mathcal{G}[e\{v / z\}]:: B, A \vdash \mathcal{F}[e\{v / z\}]:: B$ by $(A p p)$

The following technical properties are related to the propagation of footprints from the post-condition to pre-condition of typing judgments of a certain form, often useful to prove results below. In all the four next statements $\mathcal{E}, \mathcal{F}$ and $\mathcal{G}$ are assumed to be active type assertion contexts. The proof is in all cases a simple induction on typing derivations, we also give some useful justifications.

Lemma A.6. Let $A \vdash_{x} v:: \mathcal{E}[x: U]$. Then there is $C$ such that $C \vdash_{x} v:: x: U$ and $A \vdash_{x} v:: \mathcal{E}[C]$.

Lemma A.7. Let $D \vdash_{y} v:: B$ and $B<: \mathcal{F}[n: \operatorname{rd}(U)]$. Then there is $\mathcal{E}[]$ such that $D<: \mathcal{E}[n: \operatorname{rd}(U)]$ and for all $C, \mathcal{E}[C] \vdash_{y} v:: \mathcal{F}[C]$.

Intuitively, Lemma A.7 holds as a consequence of $\mathcal{F}$ being active: $n: \operatorname{rd}(U)$ is available at the front of the post-condition, and thus cannot be a residual of an usage of the value $v$, which would need to be either in the front of the context hole (contradiction), or isolated, but then the variable residual $n$ could not have escaped.

LemmA A.8. Let $A<: \mathcal{E}[n: \operatorname{rd}(U)]$. Then there are $\mathcal{F}, \mathcal{G}$ such that $A=\mathcal{F}[n: \operatorname{rd}(V)], n: \operatorname{rd}(V)<: \mathcal{G}[n: \operatorname{rd}(U)]$ and for all $C$, $\mathcal{F}[\mathcal{G}[C]]]<: \mathcal{E}[C]$. 
This last lemma, states that an active $a: \operatorname{rd}(U)$ capability in a type assertion selected by subsumption from $A$ must result from some active $a: \operatorname{rd}(V)$ capability in $A$, such that $U$ is active in $V$. This results from the distributing subtyping axioms for $\operatorname{rd}(U)$, e.g., $\operatorname{rd}\left(U_{1} \mid U_{2}\right)<: \operatorname{rd}\left(U_{1}\right) \mid \operatorname{rd}\left(U_{2}\right)$, and so on. The next lemma expresses a basic inversion principle for $B\{x / n$ :var $\}$.

LEMmA A.9. Let $B\{y / n: \operatorname{var}\}<: \mathcal{E}[n: \operatorname{rd}(U)]$ with $n$ fresh in $B$. Then there are $\mathcal{G}, \mathcal{F}$ and $V$ such that $B=\mathcal{G}[y: V], y: V<: \mathcal{F}[y: U]$, and for all $C, \mathcal{G}[\mathcal{F}[C]]\{y / n$ :var $\}<: \mathcal{E}[C]$.

The next lemma states several key inversion principles for the basic typing relation for configurations.

LEMMA A.10 (Configuration Typing Inversions). Let $\mathcal{E}[-]$ be an active context. We have

1. Let $h ; S \triangleright A$ and $A$ <: $\mathcal{E}[n: \operatorname{rd}(U)]$. Then $h(n)=v$ and there is $C$ such that $C \vdash_{x} v:: x: U$ and $h ; S \triangleright \mathcal{E}[C]$.

2. Let $h ; S \triangleright A$ and $A<: \mathcal{E}[n: \operatorname{rd}(U)$; use $]$. Then $h(n)=v$ and is $C$ s.t. $C \vdash_{x} v:: x: U$ and $h, n \mapsto v ; S \triangleright \mathcal{E}[n$ :use $C]$.

3. Let $h ; S \triangleright A$ and $A<: \mathcal{E}[C \mid n$ :use $]$. Then $h=h^{\prime}[n \mapsto u]$, and $C \vdash_{x} v:: x: U$ implies $h^{\prime}[n \mapsto v] ; S \triangleright \mathcal{E}[n: \operatorname{rd}(U)]$.

4. Let $h ; S \triangleright A$ and $A<: \mathcal{E}[n: \operatorname{wr}(\circ U) \mid C]$. Then $h=h^{\prime}, n \mapsto$ $u$, and $C \vdash_{x} v:: x: \circ U$ implies $h^{\prime}, n \mapsto v ; S \triangleright \mathcal{E}[0]$.

5. Let $h ; S \triangleright A$ and $A<: \mathcal{E}[t: \tau(U)]$. Then $S=S^{\prime} \cdot t\langle e\rangle$ and there is $C$ such that $C \vdash_{x} e:: x: U$ and $h ; S \triangleright \mathcal{E}[C]$.

Proof. In all cases, the proof is by induction on the derivation of $h ; S \triangleright A$. We detail item 1 .

1. Induction on the derivation of $h ; S \triangleright A$. We use two hole active contexts (e.g. $\mathcal{F}[-][-]$ ), defined as expected.

(Case $(E))$ not possible.

(Case $(T)$ )

$A=\mathcal{E}^{\prime}[t: \tau(T)]<: \mathcal{E}[n: \operatorname{rd}(U)], S=S^{\prime} \cdot t\langle e\rangle, h ; S^{\prime} \triangleright \mathcal{E}^{\prime}[C]$, and $C \vdash_{y} e:: y: T$, by inversion (for some $\mathcal{E}^{\prime}, S^{\prime}$, etc).

$\mathcal{E}^{\prime}[t: \tau(T)]=\mathcal{F}[t: \tau(T)][n: \operatorname{rd}(V)]<: \mathcal{E}[n: \operatorname{rd}(U)]$, for some $\mathcal{F}$ $\mathcal{E}[n: \operatorname{rd}(U)]=\mathcal{E}^{\prime \prime}[t: \tau(T)][n: \operatorname{rd}(U)]$

$\mathcal{E}^{\prime}[C]=\mathcal{F}[C][n: \operatorname{rd}(V)]<: \mathcal{E}^{\prime \prime}[C][n: \operatorname{rd}(U)]$

$n: \operatorname{rd}(V)<: \mathcal{G}[n: \operatorname{rd}(U)]$ and

$\mathcal{F}[C][\mathcal{G}[-]]<: \mathcal{E}^{\prime \prime}[C][-]$, for some $\mathcal{G}$

$\mathcal{E}^{\prime}[C]=\mathcal{F}[C][n: \operatorname{rd}(V)]<: \mathcal{F}[C][\mathcal{G}[n: \operatorname{rd}(U)]]$

$h=h^{\prime}, n \mapsto v$ and exists $D$ such that $D \vdash_{x} v:: x: U$

and $h ; S^{\prime} \triangleright \mathcal{F}[C][\mathcal{G}[D]]$, by i.h.

$h ; S \triangleright \mathcal{E}^{\prime \prime}[C][D]$, by above, and $h ; S \triangleright \mathcal{E}^{\prime \prime}[t: \tau(T)][D]$, by $(T)$

$h ; S \triangleright \mathcal{E}[D]$

(Case $(H)$ )

$h=h^{\prime \prime}, m \mapsto u$ and $h^{\prime \prime} ; S \triangleright D$ and $D \vdash_{y} u:: B$ and

$A=B\{y / m: \operatorname{var}\}<: \mathcal{E}[n: \operatorname{rd}(U)]$, by inversion.

(Subcase $m=n$ )

$B=\mathcal{B}[y: V], y: V<: \mathcal{F}[y: U]$, and $\mathcal{B}[\mathcal{F}[]]\{y / n: \operatorname{var}\}<: \mathcal{E}[]$,

by Lemma A.9.

Hence $D \vdash_{y} u:: \mathcal{B}[\mathcal{F}[y: U]]$.

$D^{\prime \prime} \vdash_{y} u:: y: U, D \vdash_{y} u:: \mathcal{B}\left[\mathcal{F}\left[D^{\prime \prime}\right]\right]$, by Lemma A.6(1)

Set $h^{\prime \prime}=h^{\prime}, u=v$. So $h=h^{\prime}, n \mapsto v$ and $D^{\prime \prime} \vdash_{x} v:: x: U$

and $D \vdash_{y} v:: \mathcal{B}\left[\mathcal{F}\left[D^{\prime \prime}\right]\right]$ and $h^{\prime} ; S \triangleright D$

Let $A^{\prime}=\mathcal{B}\left[\mathcal{F}\left[D^{\prime \prime}\right]\right]\{y / n$ :var $\}$, so $h ; S \triangleright A^{\prime}$, by $(H)$

We have $A^{\prime}<: \mathcal{E}\left[D^{\prime \prime}\right]$, so $h ; S \triangleright \mathcal{E}\left[D^{\prime \prime}\right]$, by $(S)$

(Subcase $m \neq n$ )

$B$ <: $\mathcal{F}[n: \operatorname{rd}(U)]$ where $\mathcal{F}[-]\{y / m: \operatorname{var}\}=\mathcal{E}[-]$

$D<: \mathcal{E}^{\prime}[n: \operatorname{rd}(U)]$ and $\mathcal{E}^{\prime}[C] \vdash_{y} u:: \mathcal{F}[C]$ by Lemma A.8.

$h^{\prime \prime}=h^{\prime \prime \prime}, n \mapsto v$ and exists $C$ such that $C \vdash_{x} v:: x: U$

and $h^{\prime \prime} ; S^{\prime} \triangleright \mathcal{E}^{\prime}[C]$, by i.h.

$h, S \triangleright \mathcal{F}[C]\{y / n$ :var $\}$, by $(H)$

$h, S \triangleright \mathcal{E}[C]$, by identity above
(Case (S)) By i.h.

We prove the basic type preservation and progress Theorems.

THeOREM 4.3. If $h ; S \triangleright A$ and $h ; S \rightarrow h^{\prime} ; S^{\prime}$ then $h^{\prime} ; S^{\prime} \triangleright A$.

Proof. By induction on the derivation of $h ; S \triangleright A$.

(Case $(E)$ ) not possible.

(Case $(S)$ ) We have

$h ; S \triangleright A$

$h ; S \triangleright B$ and $B<: A$, by inversion

$h^{\prime} ; S^{\prime} \triangleright B$, by ih.

$h^{\prime} ; S^{\prime} \triangleright A$, by $(S)$

(Case $(H)$ ) We have

$h_{p}, n \mapsto v ; S \triangleright C\{x / n: \operatorname{var}\}$

$h_{p} ; S \triangleright B$ and $B \vdash_{x} v:: C$, by inversion

$h_{p}^{\prime} ; S^{\prime} \triangleright B$, by ih.

$h_{p}^{\prime}, n \mapsto v ; S^{\prime} \triangleright C\{x / n$ :var $\}$, by $(H)$

(Case $(T)$ )

$h ; R \cdot t\langle e\rangle \triangleright \mathcal{E}[t: \tau(T)]$

$h ; R \triangleright \mathcal{E}[C]$ and $C \vdash_{x} e:: x: T$, by inversion

There are two subcases:

(Subcase a) $h ; R \cdot t\langle e\rangle \rightarrow h^{\prime} ; R^{\prime} \cdot t\langle e\rangle$

$h^{\prime} ; R^{\prime} \triangleright B$, by ih.

$h^{\prime} ; R^{\prime} \cdot t\langle e\rangle \triangleright \mathcal{E}[t: \tau(T)]$, by $(T)$

(Subcase b) $h ; R \cdot t\langle e\rangle \rightarrow h^{\prime} ; R^{\prime} \cdot t\left\langle e^{\prime}\right\rangle$.

We consider all possible cases for this reduction step.

(SubCase (Red let)) We have

$e=\mathcal{F}\left[\right.$ let $z=v$ in $\left.e_{1}\right]$ and $e^{\prime}=\mathcal{F}\left[e_{1}\{v / z\}\right]$

$h^{\prime}=h$ and $R^{\prime}=R$

$C \vdash_{x} \mathcal{F}\left[e_{1}\{v / z\}\right]:: x: T$, by Lemma A.5(1)

$h^{\prime} ; R^{\prime} \cdot t\left\langle e^{\prime}\right\rangle \triangleright \mathcal{E}[t: \tau(T)]$, by $(T)$

(SubCase (Red beta)) We have

$e=\mathcal{F}\left[\left(\lambda z . e_{1}\right) v\right]$ and $e^{\prime}=\mathcal{F}\left[e_{1}\{v / z\}\right]$

$h^{\prime}=h$ and $R^{\prime}=R$

$C \vdash_{x} \mathcal{F}\left[e_{1}\{v / z\}\right]:: x: T$, by Lemma A.5(2)

$h^{\prime} ; R^{\prime} \cdot t\left\langle e^{\prime}\right\rangle \triangleright \mathcal{E}[t: \tau(T)]$, by $(T)$

(SubCase (Red sel)) We have

$\left.e=\mathcal{F}[\overline{l=e}] . l_{i}\right]$ and $e^{\prime}=\mathcal{F}\left[e_{i}\right]$

$h^{\prime}=h$ and $R^{\prime}=R$

$C \vdash_{x} \mathcal{F}\left[e_{i}\right]:: x: T$, by Lemma A.5(3)

$h^{\prime} ; R^{\prime} \cdot t\left\langle e^{\prime}\right\rangle \triangleright \mathcal{E}[t: \tau(T)]$, by $(T)$

(SubCase (Red case)) We have

$e=\mathcal{F}\left[\right.$ case $l_{i}(v)$ of $\left.\overline{l(x) \rightarrow e}\right]$ and $e^{\prime}=\mathcal{F}\left[e_{i}\left\{v / x_{i}\right\}\right]$

$h^{\prime}=h$ and $R^{\prime}=R$

$C \vdash_{x} \mathcal{F}\left[e_{i}\left\{v / x_{i}\right\}\right]:: x: T$, by Lemma A.5(3)

$h^{\prime} ; R^{\prime} \cdot t\left\langle e^{\prime}\right\rangle \triangleright \mathcal{E}[t: \tau(T)]$, by $(T)$

(SubCase (Red var)) We have

$e=\mathcal{F}\left[\operatorname{var} a\right.$ in $\left.e_{1}\right]$ and $e^{\prime}=\mathcal{F}\left[e_{1}\{n / a\}\right]$

$h^{\prime}=h, n \mapsto$ nil and $R^{\prime}=R$

$C \vdash{ }_{x} \mathcal{F}\left[\operatorname{var} a\right.$ in $\left.e_{1}\right]:: x: T$, from above

$C \mid n: \operatorname{var} \vdash_{x} \mathcal{F}\left[e_{1}\{n / a\}\right]:: x: T$, by Lemma A.5(5)

$C \vdash{ }_{w}$ nil :: $w: 0 ; C$, by $(V I d)$

$h^{\prime}, R^{\prime} \triangleright C \mid n$ :var, by $(H)$

$h^{\prime} ; R^{\prime} \cdot t\left\langle e^{\prime}\right\rangle \triangleright \mathcal{E}[t: \tau(T)]$, by $(T)$

(SubCase (Red deref)) We have

$e=\mathcal{F}[a]$ and $e^{\prime}=\mathcal{F}[v], h^{\prime}=h$ and $h(a)=v$ and $R^{\prime}=R$

$C \vdash_{x} \mathcal{F}[a]:: x: T$, from above

(subcase $(a)) C$ <: $\mathcal{G}[a: \operatorname{rd}(U)]$, by Lemma A.5(7a)

$h ; R^{\prime} \triangleright \mathcal{E}[\mathcal{G}[D]]$ and $D \vdash_{z} v:: z: U$, by Lemma A.10(1)

$\mathcal{G}[D] \vdash_{x} \mathcal{F}[v]:: x: T$, by Lemma A.5(7a)

$h^{\prime} ; R^{\prime} \cdot t\left\langle e^{\prime}\right\rangle \triangleright \mathcal{E}[t: \tau(T)]$, by $(T)$

(subcase $(b)) C<: \mathcal{G}[a: r d(U)$; use $]$, by Lemma A.5(7b)

$h ; R^{\prime} \triangleright \mathcal{G}[D \mid a$ :use $]$ and $D \vdash_{z} v:: z: U$, by Lemma A.10(2)

$\mathcal{G}[D \mid a:$ use $] \vdash_{x} \mathcal{F}[v]:: x: T$, by Lemma A.5(7b)

$h^{\prime} ; R^{\prime} \cdot t\left\langle e^{\prime}\right\rangle \triangleright \mathcal{E}[t: \tau(T)]$, by $(T)$ 
(SubCase (Red assign))

$e=\mathcal{F}[a:=v]$ and $e^{\prime}=\mathcal{F}[$ nil $]$

$h=h_{p}, a \mapsto u$ and $h^{\prime}=h_{p}, a \mapsto v$ and $R^{\prime}=R$

$C \vdash_{x} \mathcal{F}[a:=v]:: x: T$, from above

(subcase $(a)) C<: \mathcal{G}[a:$ use $\mid D]$,

$\mathcal{G}[a: \operatorname{rd}(U)] \vdash_{x} \mathcal{F}[\mathbf{n i l}]:: x: T$,

$D \vdash_{y} v:: y: U$, by Lemma A.5(6a)

$h^{\prime} ; R^{\prime} \triangleright \mathcal{E}[\mathcal{G}[a: \operatorname{rd}(U)]]$, by Lemma A.10(3)

$h^{\prime} ; R^{\prime} \cdot t\left\langle e^{\prime}\right\rangle \triangleright \mathcal{E}[t: \tau(T)]$, by $(T)$

(subcase $(b)) C<: \mathcal{G}[a: \operatorname{wr}(\circ U) \mid D]$,

$\mathcal{G}[0] \vdash_{x} \mathcal{F}[$ nil] $:: x: T$,

$D \vdash_{y} v:: y: \circ U$, by Lemma A.5(6b)

$h^{\prime} ; R^{\prime} \triangleright \mathcal{E}[G[0]]$, by Lemma A.10(4)

$h^{\prime} ; R^{\prime} \cdot t\left\langle e^{\prime}\right\rangle \triangleright \mathcal{E}[t: \tau(T)]$, by $(T)$

(SubCase (Red fork)) We have

$e=\mathcal{F}\left[\right.$ fork $\left.\left(e_{1}\right)\right]$ and $e^{\prime}=\mathcal{F}[k]$, with fresh $k$

$h^{\prime}=h$ and $R^{\prime}=R \cdot k\langle e\rangle$

$C<: \mathcal{G}[D], D \vdash_{y} e_{1}:: y: U$,

$\mathcal{G}[k: \tau(U)] \vdash_{x} \mathcal{F}[k]:: x: T$, by Lemma A.5(8)

$h^{\prime} ; R \cdot k\left\langle e_{1}\right\rangle \triangleright \mathcal{E}[\mathcal{G}[k: \tau(U)]]$, by $(T)$

$h^{\prime} ; R^{\prime} \cdot t\left\langle e^{\prime}\right\rangle \triangleright \mathcal{E}[t: \tau(T)]$, by $(T)$

(SubCase (Red wait)) We have

$e=\mathcal{F}[$ wait $(k)]$ and $e^{\prime}=\mathcal{F}[v]$

$h^{\prime}=h$ and $R=R^{\prime} \cdot k\langle v\rangle$

$C<: \mathcal{G}[k: \tau(U)]$, by Lemma A.5(9)

$D \vdash_{y} v:: y: U$, and

$h ; R^{\prime} \triangleright \mathcal{E}[\mathcal{G}[D]]$, by Lemma A.10(5)

$\mathcal{G}[D] \vdash_{x} \mathcal{F}[v]:: x: T$, by Lemma A.5(9)

$h^{\prime} ; R^{\prime} \cdot t\left\langle e^{\prime}\right\rangle \triangleright \mathcal{E}[t: \tau(T)]$, by $(T)$

Progress, Theorem 4.4, is a consequence of the following.

Proposition A.11. If $h ; S \triangleright \mathcal{E}[C]$ and $C \vdash_{x} e:: D$ and live $(e)$ then $h ; S \cdot t\langle e\rangle \rightarrow h^{\prime} ; S \cdot t\left\langle e^{\prime}\right\rangle$.

Proof. By induction on the derivation of $C \vdash_{x} e:: D$. (Cases $(I d)$, (VStop), (VAbs), (VPar), (VSeq), (VShr)) Not possible.

(Case $(S u b))$

$C^{\prime} \vdash_{x} e:: D^{\prime}, C<: C^{\prime}, D^{\prime}<: D$, by inversion

$h ; S \triangleright \mathcal{E}\left[C^{\prime}\right]$ by $(S)$

$h ; S \cdot t\langle e\rangle \rightarrow h^{\prime} ; S \cdot t\left\langle e^{\prime}\right\rangle$. by ih.

(Case $(P a r)$ )

$C=C_{1}\left|C_{2}, D=D_{1}\right| C_{2}, C_{1} \vdash_{x}$ e :: $D_{1}$, by inversion

$h ; S \triangleright \mathcal{G}\left[C_{1}\right]$ where $\mathcal{G}[-]=\mathcal{E}\left[-\mid C_{2}\right]$ by $(S)$

$h ; S \cdot t\langle e\rangle \rightarrow h^{\prime} ; S \cdot t\left\langle e^{\prime}\right\rangle$. by ih.

(Case $(S e q)$ )

$C=C_{1} ; C_{2}, D=D_{1} ; C_{2}, C_{1} \vdash_{x} e:: D_{1}$, by inversion

$h ; S \triangleright \mathcal{G}\left[C_{1}\right]$ where $\mathcal{G}[-]=\mathcal{E}\left[-; C_{2}\right]$ by $(S)$

$h ; S \cdot t\langle e\rangle \rightarrow h^{\prime} ; S \cdot t\left\langle e^{\prime}\right\rangle$. by ih.

(Case $(A p p))$

$e=e_{1} e_{2}, D=x: V, C=C_{1} \mid C_{2}$,

$C_{1} \vdash_{x} e_{1}:: z: U \mapsto V, C_{2} \vdash_{x} e_{2}:: z: U$, by inversion

(Subcase $\left(\right.$ live $\left.\left.\left(e_{1}\right)\right)\right)$

$h ; S \triangleright \mathcal{G}\left[C_{1}\right]$ where $\mathcal{G}[-]=\mathcal{E}\left[-\mid C_{2}\right]$ by $(S)$

$h ; S \cdot t\left\langle e_{1}\right\rangle \rightarrow h^{\prime} ; S \cdot t\left\langle e_{1}^{\prime}\right\rangle$, by i.h.

$h ; S \cdot t\left\langle e_{1} e_{2}\right\rangle \rightarrow h^{\prime} ; S \cdot t\left\langle e_{1}^{\prime} e_{2}\right\rangle$ for evaluation context $\square e$

(Subcase $\left(\operatorname{live}\left(e_{2}\right), e_{1}=v\right)$

$h ; S \triangleright \mathcal{G}\left[C_{2}\right]$ where $\mathcal{G}[-]=\mathcal{E}\left[C_{1} \mid-\right]$ by $(S)$

$h ; S \cdot t\left\langle e_{2}\right\rangle \rightarrow h^{\prime} ; S \cdot t\left\langle e_{2}^{\prime}\right\rangle$, by i.h.

$h ; S \cdot t\left\langle v e_{2}\right\rangle \rightarrow h^{\prime} ; S \cdot t\left\langle v e_{2}^{\prime}\right\rangle$ for evaluation context $v \square$

(Subcase $\left(e_{1}, e_{2}\right.$ values) $) e_{1}=\lambda z . e_{1}^{\prime}$ and $e_{2}=v$

$h ; t\left\langle\left(\lambda z . e_{1}^{\prime}\right) v\right\rangle \rightarrow h ; t\left\langle e_{1}^{\prime}\{v / z\}\right\rangle$ by (Red beta)

(Case (Iso))

$C=\circ C_{1} \mid \cdots, D=\circ D^{\prime}$, and $C \vdash_{z} e:: D^{\prime}$, by inversion

$h ; S \cdot t\langle e\rangle \rightarrow h^{\prime} ; S \cdot t\left\langle e^{\prime}\right\rangle$. by ih.

(Case $(A n d))$
$D=D_{1} \& D_{2}$, and $C \vdash_{z} e:: D_{1}$, and $C \vdash_{z} e:: D_{2}$, by inversion $h ; S \cdot t\langle e\rangle \rightarrow h^{\prime} ; S \cdot t\left\langle e^{\prime}\right\rangle$. by ih.

(Case $(\mathrm{Sel})$ )

$e$ is $e_{s} . l, D=z: l: T$, and $C \vdash_{z} e_{s}:: z: T$, by inversion

(Subcase $\left(\right.$ live $\left.\left.\left(e_{s}\right)\right)\right)$

$h ; S \cdot t\left\langle e_{s}\right\rangle \rightarrow h^{\prime} ; S \cdot t\left\langle e_{s}^{\prime}\right\rangle$. by ih.

$\left.h ; S \cdot t\left\langle e_{s} . l\right)\right\rangle \rightarrow h^{\prime} ; S \cdot t\left\langle e_{s}^{\prime} . l\right\rangle$ for evaluation context $\square . l$

(Subcase $\left(e_{s}=v\right)$ )

$v=\left[\ldots l=e_{l} \ldots\right]$

$h ; S \cdot t\left\langle\left[\ldots l=e_{l} \ldots\right] . l\right\rangle \rightarrow h^{\prime} ; S \cdot t\left\langle e_{l}\right\rangle$ by $(\operatorname{Red} \operatorname{del})$

(Case (Case))

$e=$ case $e_{c}$ of $l(x) \rightarrow e^{\prime}, C=C_{1} \mid C_{2}$,

$C_{1} \vdash_{y} e_{c}:: y: \oplus_{l \in I} l: T_{l}$ and $x_{i}: T_{i} \mid C_{2} \vdash_{z} e_{i}^{\prime}:: C$, by inversion

(Subcase $\left(\right.$ live $\left.\left.\left(e_{c}\right)\right)\right)$

$h ; S \triangleright \mathcal{G}\left[C_{1}\right]$ where $\mathcal{G}[-]=\mathcal{E}\left[-\mid C_{1}\right]$ by $(S)$

$h ; S \cdot t\left\langle e_{c}\right\rangle \rightarrow h^{\prime} ; S \cdot t\left\langle e_{c}^{\prime}\right\rangle$. by ih.

$h ; t\left\langle\right.$ case $e_{c}$ of $\left.l(x) \rightarrow e^{\prime}\right\rangle \rightarrow h^{\prime} ; t\left\langle\right.$ case $e_{c}^{\prime}$ of $\left.l(x) \rightarrow e^{\prime}\right\rangle$

for evaluation context case $\square$ of $l(x) \rightarrow e^{\prime}$

(Subcase $\left(e_{c}=v\right)$ )

$e_{c}=l_{i}\left(v^{\prime}\right)$

$h ; t\left\langle\right.$ case $l_{i}\left(v^{\prime}\right)$ of $\left.l(x)\right\rangle \rightarrow t\left\langle e^{\prime} \rightarrow h^{\prime} ; e_{i}^{\prime}\left\{v^{\prime} / x_{i}\right\}\right\rangle$

by (Red case)

(Case (Option))

$e=l_{i}\left(e_{c}\right), D=\oplus_{l \in I} l: T_{l}, C \vdash_{z} e^{\prime}:: T_{i}$, by inversion

live $\left(e_{c}\right)$

$h ; S \cdot t\left\langle e_{c}\right\rangle \rightarrow h^{\prime} ; S \cdot t\left\langle e_{c}^{\prime}\right\rangle$. by ih.

$h ; S \cdot t\left\langle l\left(e_{c}\right)\right\rangle \rightarrow h^{\prime} ; S \cdot t\left\langle l\left(e_{c}^{\prime}\right)\right\rangle$ for evaluation context $l(\square)$

(Case $($ Let $))$

$e$ is let $y=e_{1}$ in $e_{2}$, and $C \vdash_{y} e_{1}:: C^{\prime}$

and $C^{\prime} \vdash_{x} e_{2}:: D$, by inversion

(Subcase live $\left(e_{1}\right)$ )

$h ; S \cdot t\left\langle e_{1}\right\rangle \rightarrow h^{\prime} ; S \cdot t\left\langle e_{1}^{\prime}\right\rangle$, by ih.

$h ; S \cdot t\left\langle\right.$ let $y=e_{1}$ in $\left.e_{2}\right\rangle \rightarrow h^{\prime} ; S \cdot t\left\langle\right.$ let $y=e_{1}^{\prime}$ in $\left.e_{2}\right\rangle$

for evaluation context let $y=\square$ in $e_{2}$

(Subcase $e_{1}=v$ )

$h ; S \cdot t\left\langle\right.$ let $y=v$ in $\left.e_{2}\right\rangle \rightarrow h^{\prime} ; S \cdot t\left\langle e_{2}\{x / y\}\right\rangle$ by (Red let)

(Case $(\operatorname{Var})$ )

$e$ is var $a$ in $e_{v}$, by inversion

$h ; S \cdot t\langle e\rangle \rightarrow h^{\prime} ; S \cdot t\left\langle e^{\prime}\right\rangle$ by (Red var)

(Case $(R d V B))$

$e=a, C=a: \operatorname{rd}(U)$ and $D=z: U$, by inversion

$h(a)=w$, by A.10 Lemma (1)

$h ; S \cdot t\langle e\rangle \rightarrow h^{\prime} ; S \cdot t\left\langle e^{\prime}\right\rangle$ by (Red deref)

(Case $(R d V F))$

$e=a, C=a: \operatorname{rd}(U)$; use, $D=z: U \mid a$ :use, by inversion

$h(a)=w$, by Lemma A.10 (2)

$h ; S \cdot t\langle e\rangle \rightarrow h^{\prime} ; S \cdot t\left\langle e^{\prime}\right\rangle$ by (Red deref)

(Case $(W r V F))$

$e$ is $a:=u$ and $C \vdash_{w} u:: w: \circ U \mid a: w r(\circ U)$, by inversion

$C<: \mathcal{F}[a: \operatorname{wr}(\circ U)]$

$h(a)=w$, by Lemma A.10(4)

$h ; S \cdot t\langle e\rangle \rightarrow h^{\prime} ; S \cdot t\left\langle e^{\prime}\right\rangle$ by (Red assign)

(Case $(W r V B))$

$e$ is $a:=u$ and $C \vdash_{w} u:: w: U \mid a$ :use, by inversion

$C<: \mathcal{F}[a:$ use $], h(a)=w$, by Lemma A.10(3)

$h ; S \cdot t\langle e\rangle \rightarrow h^{\prime} ; S \cdot t\left\langle e^{\prime}\right\rangle$ by (Red assign)

THEOREM 4.4. If $h ; S \triangleright A$ and live $(S)$ then $h ; S \rightarrow h^{\prime} ; S^{\prime}$.

Proof. By induction on the derivation of $h ; S \triangleright A$ we can show that there is $h^{\prime} ; S^{\prime} \triangleright \mathcal{E}[C]$ (with $S=S^{\prime} \cdot S^{\prime \prime}$ and $h=h^{\prime}, h^{\prime \prime}$ ) and $C \vdash_{x} e:: D$ where live $(e)$. We conclude by Proposition A.11.

We now extend our results to the full language with synchronization constructs and invariant-based separation. The reduction cases of Lemma A.5 are extended to the appropriate judgment form 
$A \vdash^{\iota} e:: B$ as stated in Lemma A.13 below. Recall that a lock invariant is a heap assertion $R$ such that $R<$ : $\circ R$ (Section 4.5). An useful commutation property is the following:

LEMMA A.12. Let $R$ be a lock invariant. If $A \vdash_{x}^{\iota} v:: R \mid B$ then there is $C$ such that $A<: C \mid R$ and $C \vdash_{x}^{\iota} v:: B$.

LeMmA A.13. Let $\mathcal{F}$ be an evaluation context. Then

1. If $A \vdash_{x}^{\iota} \mathcal{F}[$ let $z=v$ in $e]:: B$ then $A \vdash_{x}^{\iota} \mathcal{F}[e\{v / z\}]:: B$;

2. If $A \vdash_{x}^{\iota} \mathcal{F}[(\lambda z . e) v]:: B$ then $A \vdash_{x}^{\iota} \mathcal{F}[e\{v / z\}]:: B$;

3. If $A \vdash_{x}^{\iota} \mathcal{F}\left[[\overline{l=e}] . l_{i}\right]:: B$ then $A \vdash_{x}^{\iota} \mathcal{F}\left[e_{i}\right]:: B$;

4. If $A \vdash_{x}^{\iota} \mathcal{F}\left[\right.$ case $l_{i}(v)$ of $\left.\overline{l(x) \rightarrow e}\right]:: B$ then $A \vdash_{x}^{\iota} \mathcal{F}\left[e_{i}\left\{v / x_{i}\right\}\right]:: B$

5. If $A \vdash_{x}^{\iota} \mathcal{F}[$ var $a$ in $e]:: B$ then exists $C$ and lock invariant $R$ such that $A<: C \mid R$ and $C \mid n$ :var $\vdash_{x}^{\iota\{R / n\}} \mathcal{F}[e\{n / a\}]: B$;

6. If $A \vdash_{x}^{\iota} \mathcal{F}[n:=v]:: B$ then there is $C$ such that either

(a) $A<: \mathcal{E}[n$ :use | $C]$ and $\mathcal{E}[n: \operatorname{rd}(U)] \vdash_{x}^{\iota} \mathcal{F}[$ nil $]:: B$ and $C \vdash_{z}^{\iota} v:: z: U$.

(b) $A<: \mathcal{E}[n: \mathrm{wr}(\circ U) \mid C]$ and $\mathcal{E}[0] \vdash_{x}^{\iota} \mathcal{F}[$ nil $]:: B$ and $C \vdash_{z}^{\iota} v:: z: \circ U$.

7. If $A \vdash_{x}^{\iota} \mathcal{F}[n]:: B$ then either

(a) $A$ <: $\mathcal{E}[n: \operatorname{rd}(U)]$ and for all $C \vdash_{z}^{\iota} v:: z: U$ we have $\mathcal{E}[C] \vdash_{x}^{\iota} \mathcal{F}[v]:: B$;

(b) $A<: \mathcal{E}[n: \operatorname{rd}(U)$; use $]$ and for all $C \vdash_{z}^{\iota} v:: z: U$ we have $\mathcal{E}[n$ :use $\mid C] \vdash_{x}^{\iota} \mathcal{F}[v]:: B$;

8. If $A \vdash_{x}^{\iota} \mathcal{F}[$ fork $(e)]:: B$ then $A<: \mathcal{E}[C]$ and $C \vdash_{z}^{\iota} e:: z: U$ and $\mathcal{E}[t: \tau(U)] \vdash_{x}^{\iota} \mathcal{F}[t]:: B$, for fresh $t$

9. If $A \vdash_{x}^{\iota} \mathcal{F}[\operatorname{wait}(t)]:: B$ then $A<: \mathcal{E}[t: \tau(U)]$ and for all $C \vdash_{z}^{\iota} v:: z: U$ we have $\mathcal{E}[C] \vdash_{x}^{\iota} \mathcal{F}[v]:: B ;$

10. If $A \vdash_{x}^{\iota} \mathcal{F}[\operatorname{sync}(n) e]:: B$ then $A \mid \iota(n) \vdash_{x}^{\iota} \mathcal{F}[s y(n) e]:: B$.

11. If $A \vdash_{x}^{\iota} \mathcal{F}[s y(n) v]:: B$ then $A<: C \mid \iota(n)$ and $C \vdash_{x}^{\iota} \mathcal{F}[v]:: B$.

Proof. By induction on the typing derivations. We detail some cases of 5 and 11 .

5. Induction on the derivation of $A \vdash_{x}^{\iota} \mathcal{F}[\operatorname{var} a$ in $e]:: B$.

(Case $($ Par $)$ )

$A=A_{1}\left|D, B=B_{1}\right| D$

$A_{1} \vdash_{x}^{\iota} \mathcal{F}[\operatorname{var} a$ in $e]:: B_{1}$, by inversion

Exists $C$ and lock invariant $R$ such that $A_{1}<: C \mid R$

and $C \mid n: \operatorname{var} \vdash_{x}^{\iota\{R / n\}} \mathcal{F}[e\{n / a\}]:: B_{1}$, by i.h.

$A<: C|R| D$

$D|C| n: \operatorname{var} \vdash_{x}^{\iota\{R / n\}} \mathcal{F}[e\{n / a\}]:: B$, by (Par)

(Case $($ Var $)$ )

$A<: A_{1} \mid R$, with lock invariant $R$

$A_{1} \mid n: \operatorname{var} \vdash_{x}^{\iota\{R / a\}} \mathcal{F}[e\{n / a\}]:: B$

by inversion, and fresh renaming

(Case $(S e q)$ )

$A=A_{1} ; D, B=B_{1} ; D$

$A_{1} \vdash_{x}^{\iota} \mathcal{F}[$ var $a$ in $e]:: B_{1}$, by inversion

Exists $C$ and lock invariant $R$ such that $A_{1}<: C \mid R$ and $C \mid n: \operatorname{var} \vdash_{x}^{\iota\{R / n\}} \mathcal{F}[e\{n / a\}]:: B_{1}$, by i.h.

$(C \mid n: \operatorname{var}) ; D \vdash_{x}^{\iota\{R / n\}} \mathcal{F}[e\{n / a\}]:: B$, by $(S e q)$

$C|n: \operatorname{var}| D \vdash_{x}^{\iota\{R / n\}} \mathcal{F}[e\{n / a\}]:: B$, by $(S u b)$

$A<:(C \mid R) ; D<: C|D| R$ (since $R<: \circ R$ )

11. Induction on the derivation of $A \vdash_{x}^{\iota} \mathcal{F}[s y(n) v]:: B$.

(Case (Par))

$A=A_{1}\left|C, B=B_{1}\right| C$

$A_{1} \vdash_{x}^{\iota} \mathcal{F}[s y(n) v]:: B_{1}$, by inversion

$A_{1}<: D \mid \iota(n)$ and $D \vdash_{x}^{\iota} \mathcal{F}[v]:: B_{1}$, by i.h.

$A<: D|C| \iota(n)$

$D \mid C \vdash_{x}^{\iota} \mathcal{F}[v]:: B$, by $(P a r)$

(Case $($ Let $))$

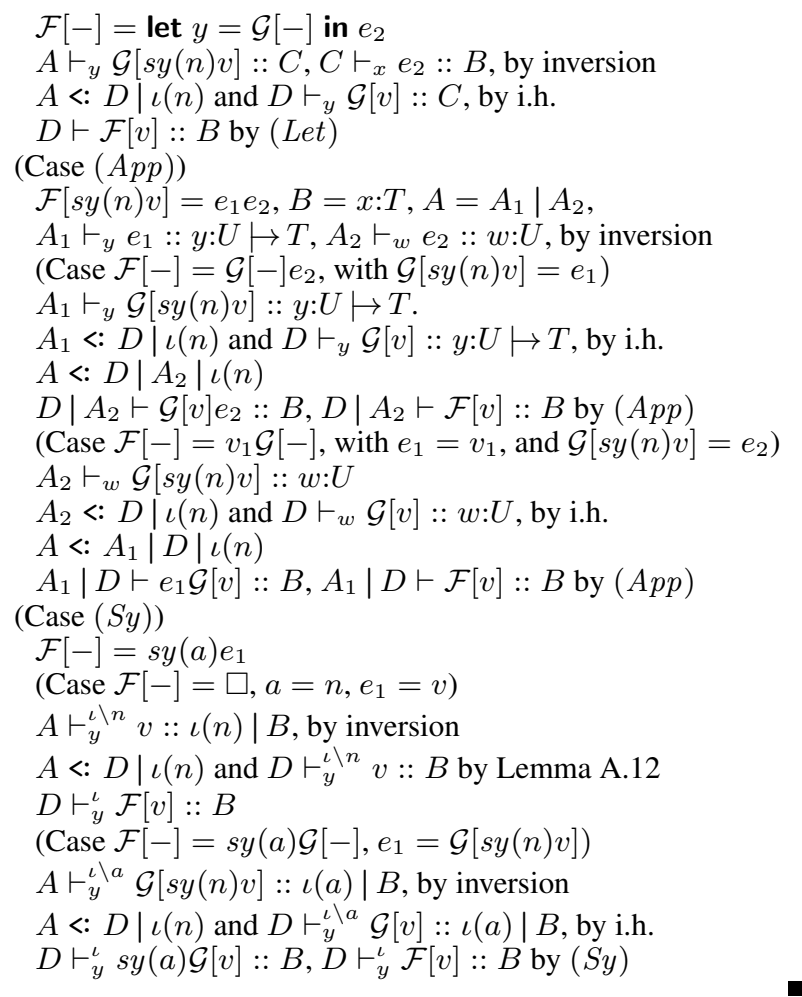

All properties listed in Lemma A.5 also hold of the configuration typing $h ; S \triangleright_{\iota} A$ (see Section A.2).

Lemma A.14 pinpoints the interplay between the state of variable $n$ lock and its invariant assertion $\iota(n)$ at the level of typing. Acquiring the lock transfers ownership of the invariant from the lock to post-condition (1), and releasing the lock transfers ownership of the invariant back to the lock (2).

Lemma A.14. Let $h ; S \triangleright_{\iota} A, n_{k} \mapsto v \in h$, and no thread in $S$ holds lock $n$ (no thread in $S$ has an active sy (n)e subexpression).

1. If $k=0$ then $h\left[n_{-1}\right] ; S \triangleright_{\iota} A \mid \iota(n)$.

2. If $A<: C \mid \iota(n)$, and $k=-1$ then $h\left[n_{0}\right] ; S \triangleright_{\iota} C$.

Proof. Induction on the configuration typing derivation, the base case being the step that introduces $n$ in the heap $h$. In (1), we simply replace $(H U)$ by $(H L)$ in such step. In (2) we move $\iota(n)$ back over all heap cells in $h$ (using Lemma A.12), and over all threads in $S$ (using the assumption), until the step where $n$ is introduced (base case), where we replace the instance of rule $(H L)$ by $(H U)$.

We now state and prove our final main result, the type preservation property for the full language with synchronization primitives and invariant-based reasoning.

Theorem 4.5. If $h ; S \triangleright_{\iota} A$ and $h ; S \rightarrow h^{\prime} ; S^{\prime}$ then $h^{\prime} ; S^{\prime} \triangleright_{\iota} A$.

Proof. By induction on the derivation of $h ; S \triangleright_{\iota} A$. We detail some key cases.

(Case $(H U)$ ) We have

$h_{p}, n_{0} \mapsto v ; S \triangleright_{\iota\{R / n\}} C\{x / n$ :var $\}$

$h_{p} ; S \triangleright_{\iota} B \mid R$ and $B \vdash_{x}^{\iota} v:: C$, by inversion

$h_{p}^{\prime} ; S^{\prime} \triangleright_{\iota} B \mid R$, by ih.

$h_{p}^{\prime}, n_{0} \mapsto v ; S^{\prime} \triangleright_{\iota\{R / n\}} C\{x / n$ :var $\}$, by $(H U)$

(Case $(H L))$ We have

$h_{p}, n_{-1} \mapsto v ; S \triangleright_{\iota\{R / n\}} C\{x / n$ :var $\}$

$h_{p} ; S \triangleright_{\iota} B$ and $B \vdash_{x}^{\iota} v:: C$, by inversion

$h_{p}^{\prime} ; S^{\prime} \triangleright_{\iota} B$, by ih.

$h_{p}^{\prime}, n_{-1} \mapsto v ; S^{\prime} \triangleright_{\iota\{R / n\}} C\{x / n$ :var $\}$, by $(H L)$ 


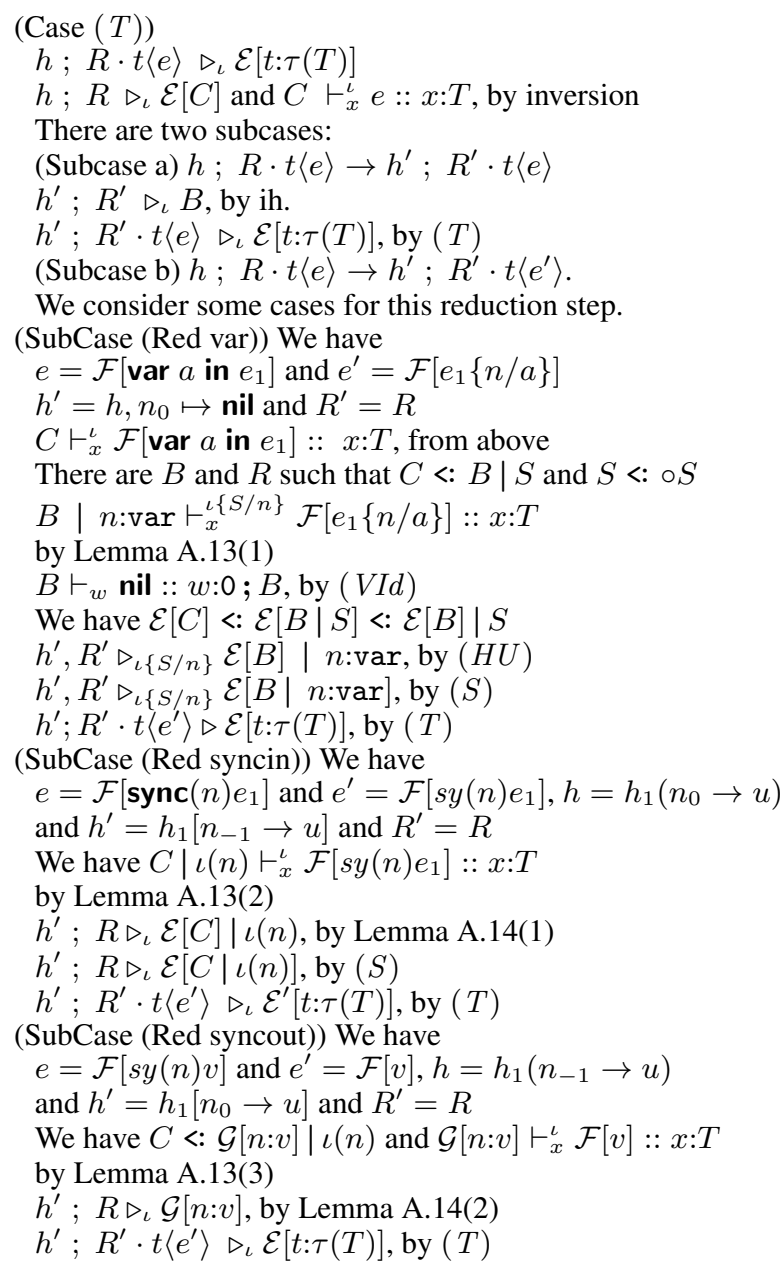

\section{A.6 Progress up to locking}

A progress property can be stated and proved for the full type system with synchronization primitives, by filtering out the situations where progress is hindered by the impossibility of acquiring a lock. Apart from this, well-typed programs are stuck free, and absent of races in the sense explained in Sections 4.4 and A.3.

We say that an expression $e$ is live up to locking in heap $h$, noted $\operatorname{liveh}(e, h)$, if it is neither a value, nor of the form $\mathcal{F}\left[\operatorname{sync}(a) e_{1}\right]$ and $a_{-1} \mapsto v \in h$ for some evaluation context $\mathcal{F}$.

A multiset $S$ of threads is live up to locking in heap $h$, noted liveh $(S, h)$ if there is some thread $t\langle e\rangle$ in $S$ such that liveh $(e, h)$. We can then prove the following "progress up to locking property".

Theorem A.15. If $h ; S \triangleright_{\iota} A$ and liveh $(S, h)$ then $h ; S \rightarrow h^{\prime} ; S^{\prime}$.

THEOREM A.16. If $h ; S \triangleright_{\iota} \mathcal{E}[C]$ and $C \vdash_{x}^{\iota} e:: D$ and liveh $(e, h)$ then $h ; S \cdot t\langle e\rangle \rightarrow h^{\prime} ; S \cdot t\left\langle e^{\prime}\right\rangle$.

Proof. Identical to proof of Theorem 4.4, by induction on the typing derivation $C \vdash_{x}^{\iota} e:: D$. We show a few interesting cases.

(Case $($ Sync) $)$

$e=\operatorname{sync}(a) e_{1}, \iota(a)\left|C \vdash_{x}^{\iota \backslash a} e_{1}:: \iota(a)\right| D$, by inversion

Since liveh $(e, h)$, we have $a_{0} \mapsto u \in h$.

$h ; t\left\langle\operatorname{sync}(a) e_{1}\right\rangle \rightarrow h\left[a_{-1}\right] ; t\left\langle s y(a) e_{1}\right\rangle$ by (Red syncin)

(Case $(S y)$ )

$e=s y(a) e_{1}, C \vdash_{x}^{\iota \backslash a} e_{1}:: \iota(a) \mid D$, by inversion

(Subcase $\left.\left(\operatorname{liveh}\left(e_{1}, h\right)\right)\right)$

$h ; S \cdot t\left\langle e_{1}\right\rangle \rightarrow h^{\prime} ; S \cdot t\left\langle e_{1}^{\prime}\right\rangle$, by i.h. $h ; S \cdot t\left\langle s y(a) e_{1}\right\rangle \rightarrow h^{\prime} ; S \cdot t\left\langle s y(a) e_{1}^{\prime}\right\rangle$

for evaluation context $s y(a) \square$

(Subcase $\left(e_{1}\right.$ value $\left.)\right)$

$e_{1}=v$

$h ; t\langle s y(a) v\rangle \rightarrow h\left[a_{0}\right] ; t\langle v\rangle$

where $a_{-1} \mapsto u \in h$, by (Red syncout)

\section{A.7 Subtyping congruences}

As discussed in Section 4.1, we have omitted in Figure 3 the rules expressing subtyping congruences. All type operators satisfy the expected covariant subtyping congruence principles, except $(-) \mapsto(-)$ and $\operatorname{wr}(-)$ which are contravariant, e.g.,

$$
\begin{array}{ccc}
\frac{A<: A^{\prime} \quad B<: B^{\prime}}{A ; B<: A^{\prime} ; B^{\prime}} & \frac{U<: V}{\operatorname{rd}(U)<: \operatorname{rd}(V)} \\
\frac{A^{\prime}<: A}{A \mapsto B} B^{\prime} & \frac{V<: U}{\operatorname{tr}(U)<: \operatorname{wr}(V)}
\end{array}
$$

\section{A.8 Some Detailed Typing Derivations}

We complement the presentation in Section 4.3 and show in detail (decomposed in Figures 9, 10, 11, 12, and 13) the complete typing derivation for the motivating collection example presented in Section 2. The derivation for the atomic cell is shown in Figure 14. 


$$
\begin{aligned}
& 0 \vdash_{y}^{\iota, \eta} v:: 0 \text { (VStop) } \quad x: U \vdash_{y}^{\iota, \eta} x:: y: U(I d) \quad \frac{\circ A_{1}|\ldots| \circ A_{n} \vdash_{x}^{\iota, \eta} e:: B}{\circ A_{1}|\ldots| \circ A_{n} \vdash_{x}^{\iota, \eta} e:: \circ B}(\text { Iso }) \frac{! A_{1}|\ldots| ! A_{n} \vdash_{x}^{\iota, \eta} v:: B}{! A_{1}|\ldots| ! A_{n} \vdash_{x}^{\iota, \eta} v:: ! B}(V S h r) \\
& \frac{A<: A^{\prime} \quad A^{\prime} \vdash_{x}^{\iota, \eta} e:: B^{\prime} \quad B^{\prime}<: B}{A \vdash_{x}^{\iota, \eta} e:: B}(S u b) \frac{A \vdash_{y}^{\iota, \eta} e:: B \quad A \vdash_{y}^{\iota, \eta} e:: C}{A \vdash_{y}^{\iota, \eta} e:: B \& C}(A n d) \frac{A \vdash_{x}^{\iota, \eta} e_{1}:: B \quad B \vdash_{y}^{\iota, \eta} e_{2}:: C}{A \vdash_{y}^{\iota, \eta} \text { let } x=e_{1} \text { in } e_{2}:: C}(\text { Let }) \\
& \frac{A \vdash_{x}^{\iota, \eta} e:: B}{A ; C \vdash_{x}^{\iota, \eta} e:: B ; C}(S e q) \quad \frac{A \vdash_{x}^{\iota, \eta} e:: B}{A\left|C \vdash_{x}^{\iota, \eta} e:: B\right| C}(P a r) \frac{A \vdash_{x}^{\iota, \eta} e_{1}:: x: U \mapsto T \quad B \vdash_{y}^{\iota, \eta} e_{2}:: y: U}{A \mid B \vdash_{z}^{\iota, \eta} e_{1} e_{2}:: z: T}(A p p) \\
& \frac{A \vdash_{x}^{\iota, \eta} e:: x: U}{A \vdash_{x}^{\iota, \eta}[\ldots l=e \ldots]:: x: l: U}(\text { Tuple }) \quad \frac{A \vdash_{x}^{\iota, \eta} e:: x: l: T}{A \vdash_{x}^{\iota, \eta} e . l:: x: T}(\text { Sel }) \quad \frac{A \mid x: U \vdash_{z}^{\iota, \eta} e:: z: T}{A \vdash_{z}^{\iota, \eta} \lambda x . e:: z: U \mapsto T}(\text { VAbs }) \\
& \frac{A \vdash_{y}^{\iota, \eta} e::\left(y: \oplus_{l \in I} l: T_{l}\right) \quad x_{l}: T_{l} \mid B \vdash_{z}^{\iota, \eta} e_{l}:: C}{A \mid B \vdash_{z}^{\iota, \eta} \text { case } e \text { of } \overline{l(\vec{x}) \rightarrow e}:: C} \text { (Case) } \frac{A \vdash_{z}^{\iota, \eta} e:: z: T_{m} \quad(m \in I)}{A \vdash_{z}^{\iota, \eta} l_{m}(e):: z: \oplus_{l \in I} l: T_{l}} \text { (Option) } \\
& \frac{A \vdash_{y}^{\iota, \eta} v:: C \quad B \vdash_{y}^{\iota, \eta} v:: D}{A ; B \vdash_{y}^{\iota, \eta} v:: C ; D}(\text { VSeq }) \quad \frac{A \vdash_{y}^{\iota, \eta} v:: C \quad B \vdash_{y}^{\iota, \eta} v:: D}{A\left|B \vdash_{y}^{\iota, \eta} v:: C\right| D} \text { (VPar) } \\
& \frac{A<: B|R \quad a: \operatorname{var}| B \vdash_{x}^{\iota\{R / a\}, \eta} e:: C}{A \vdash_{x}^{\iota, \eta} \operatorname{var} a \text { in } e:: C}(\operatorname{Var}) \frac{\iota(a)\left|A \vdash_{x}^{\iota \backslash a, \eta} e:: \iota(a)\right| B}{A \vdash_{x}^{\iota, \eta} \operatorname{sync}(a) e:: B}(\text { Sync }) \frac{A \vdash_{x}^{\iota \backslash a, \eta} e:: \iota(a) \mid B}{A \vdash_{x}^{\iota, \eta} \operatorname{sy}(a) e:: B}(S y) \\
& \frac{A \vdash_{z}^{\iota, \eta} v:: z: U \mid a: \text { use }}{A \vdash_{z}^{\iota, \eta} a:=v:: a: \operatorname{rd}(U)}(W r V B) \quad \frac{A \vdash_{z}^{\iota, \eta} v:: z: \circ U \mid a: \operatorname{wr}(\circ U)}{A \vdash_{z}^{\iota, \eta} a:=v:: 0}(W r V F) \\
& a: \operatorname{rd}(U) \vdash_{x}^{\iota, \eta} a:: x: U(R d V B) \quad a: \operatorname{rd}(U) ; \text { use } \vdash_{x}^{\iota, \eta} a:: x: U \mid a: \text { use }(R d V F) \\
& \frac{A \vdash_{x}^{\iota, \eta} e:: x: T}{A \vdash_{x}^{\iota, \eta} \text { fork } e:: x: \tau(T)}(\text { Fork }) \quad \frac{A \vdash_{x}^{\iota, \eta} e:: x: \tau(T)}{A \vdash_{x}^{\iota, \eta} \text { wait } e:: x: T} \text { (Wait) } \\
& \frac{\eta(X)=A}{A \vdash_{x}^{\iota, \eta} v:: X}(\text { VRecVar }) \quad \frac{A \vdash_{x}^{\iota, \eta}\{X / A\}}{A \vdash_{x}^{\iota, \eta} v:: \operatorname{rec}(X) B}(\operatorname{VRec}) \quad \frac{\eta(Z)=\left(A \vdash_{x} B\right)}{A \vdash_{x}^{\iota, \eta} Z:: B}(\operatorname{Rec} \operatorname{Var}) \quad \frac{A \vdash_{x}^{\iota, \eta\left\{Z /\left(A \vdash_{x} B\right)\right\}} e:: B}{A \vdash_{x}^{\iota, \eta} \operatorname{rec}(Z) e:: B}(\operatorname{Rec})
\end{aligned}
$$

Figure 8. Typing Rules (Summary). 


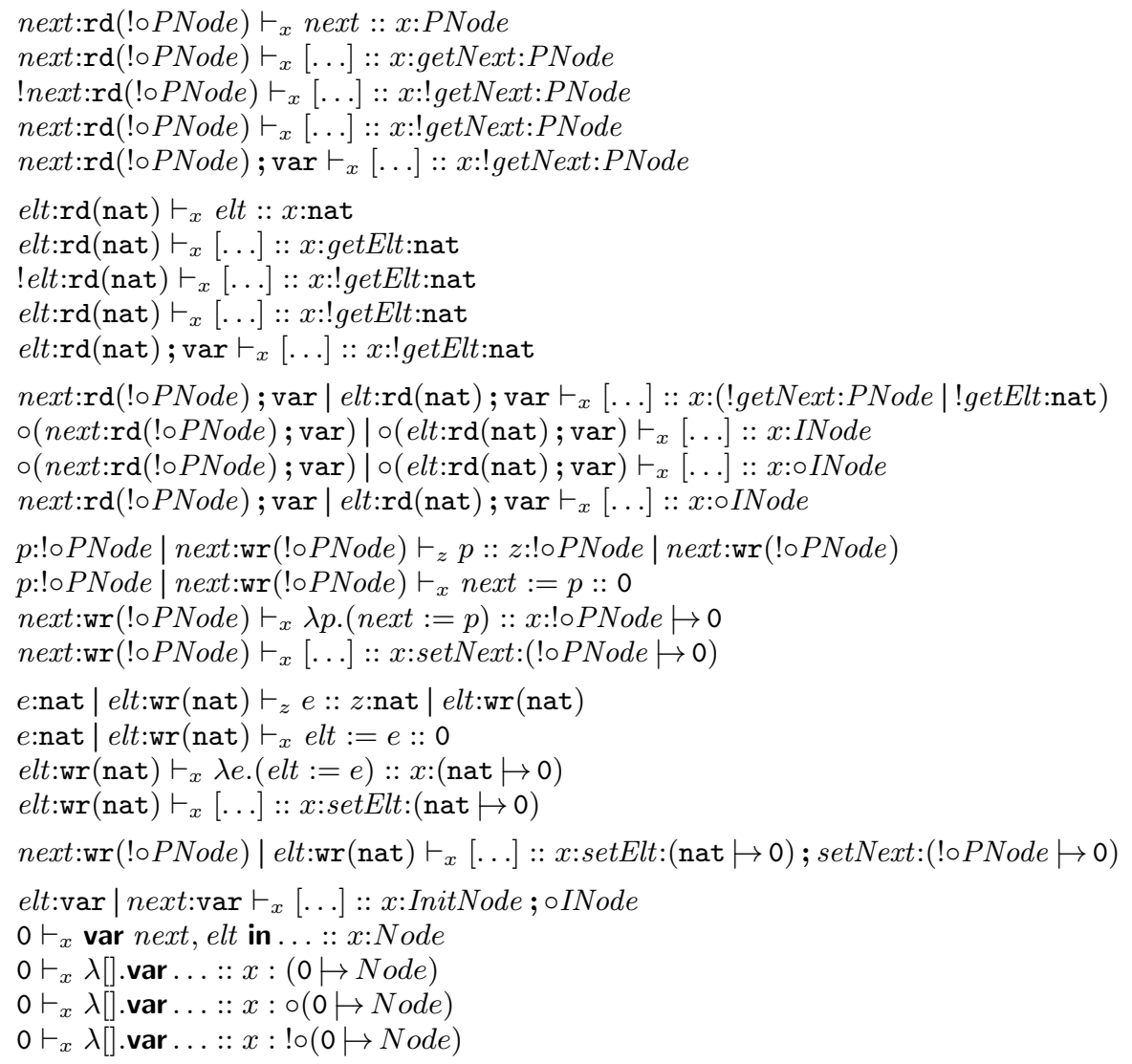

by (RdVB) and (Sub)

by (Tuple) on 1 .

by (Sub) on 2. and (VShr)

by $(\mathrm{Sub})$ on 3 .

by (Sub) on 4 .

by (RdVB)

by (Tuple) on 6.

by (Sub) on 7. and (VShr)

by (Sub) on 8 .

by $(\mathrm{Sub})$ on 9 .

by (Par) on 10. and 5 .

by (Sub) on 11 .

by (Iso) on 12 .

by (Sub) on 13 .

by (Id) and (Par) by (WrVF) on 15. by (VAbs) on 16. by (Tuple) on 17.

by (Id) and (Par) by (WrVF) on 19. by (VAbs) on 20. by (Tuple) on 21 .

by (VSeq) on 22. and 18., and (Sub) by (VSeq) on 23. and 14., and (Sub) by (Var) on 24 .

by (VAbs) on 25. by (Iso) on 26. by (VShr) on 27.

N.B. nat is a shared isolated type, so nat <: !onat, so that nat <: nat $\mid$ nat and nat <: onat.

Figure 9. Typing derivation for newNode function 


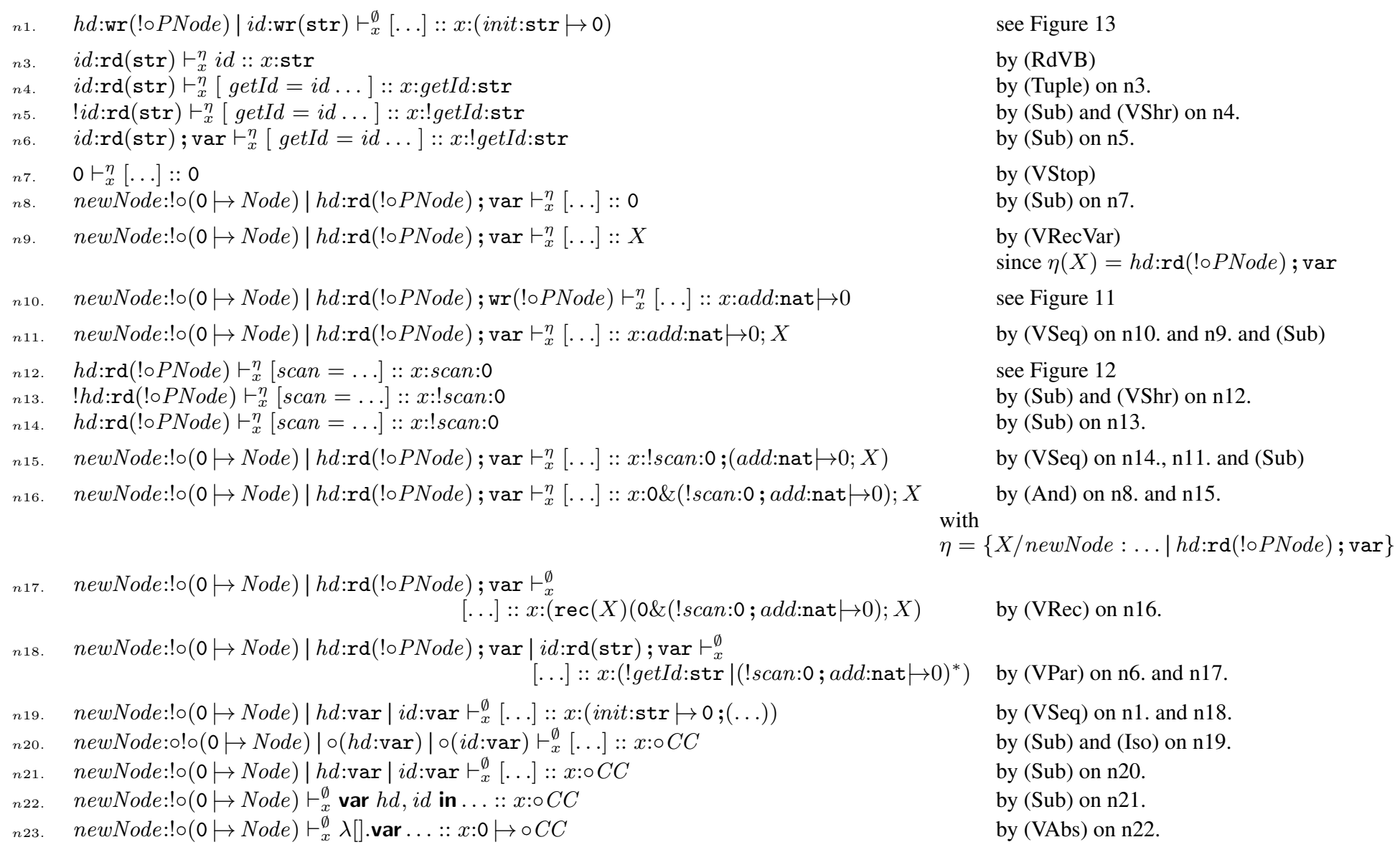

Figure 10. Typing derivation for function new Coll 


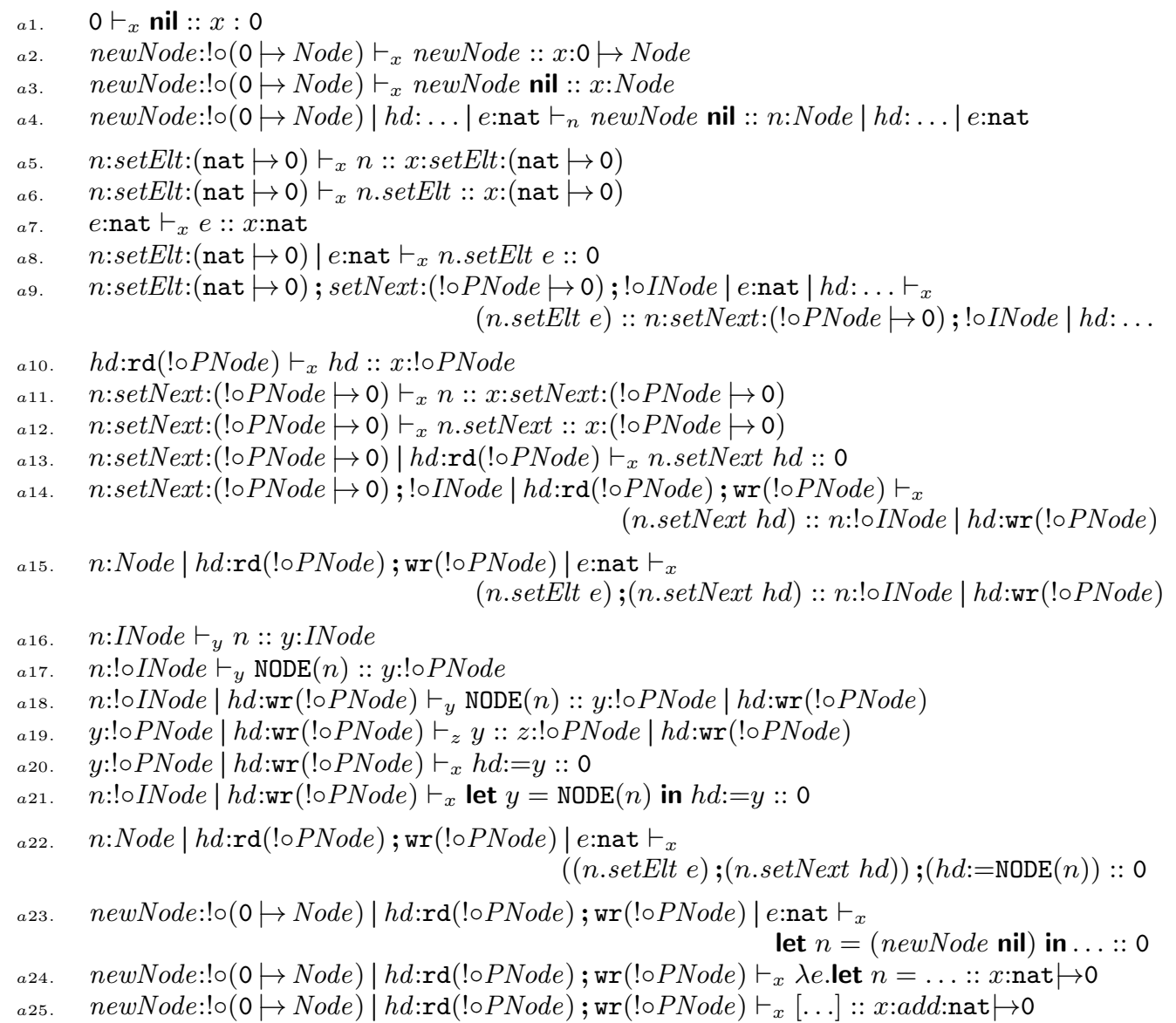

by (VStop)

by (Id) and (Sub)

by (App) on a1. and a2.

by (Par) on a3.

by (Id)

by (Sel) on a5

by (Id)

by (App) on a6. and a7.

by (Seq) and (Par) on a8.

by (RdVB)

by (Id)

by (Sel) on a11.

by (App) on a10. and a12.

by (Seq), (Par), and (Sub) on a13.

by (Let) on a9. and a14.

by (Id)

by (Option), (Sub), (Iso), and (VShr) on a16.

by (Par) on a17.

by (Id) and (Par)

by (WrVF) on a19.

by (Let) on a18. and a20.

by (Let) on a15.and a21.

by (Let) on a4. and a22.

by (Abs) on a23.

by (Tuple) on a24.

Figure 11. Typing derivation for "method" $a d d$ 


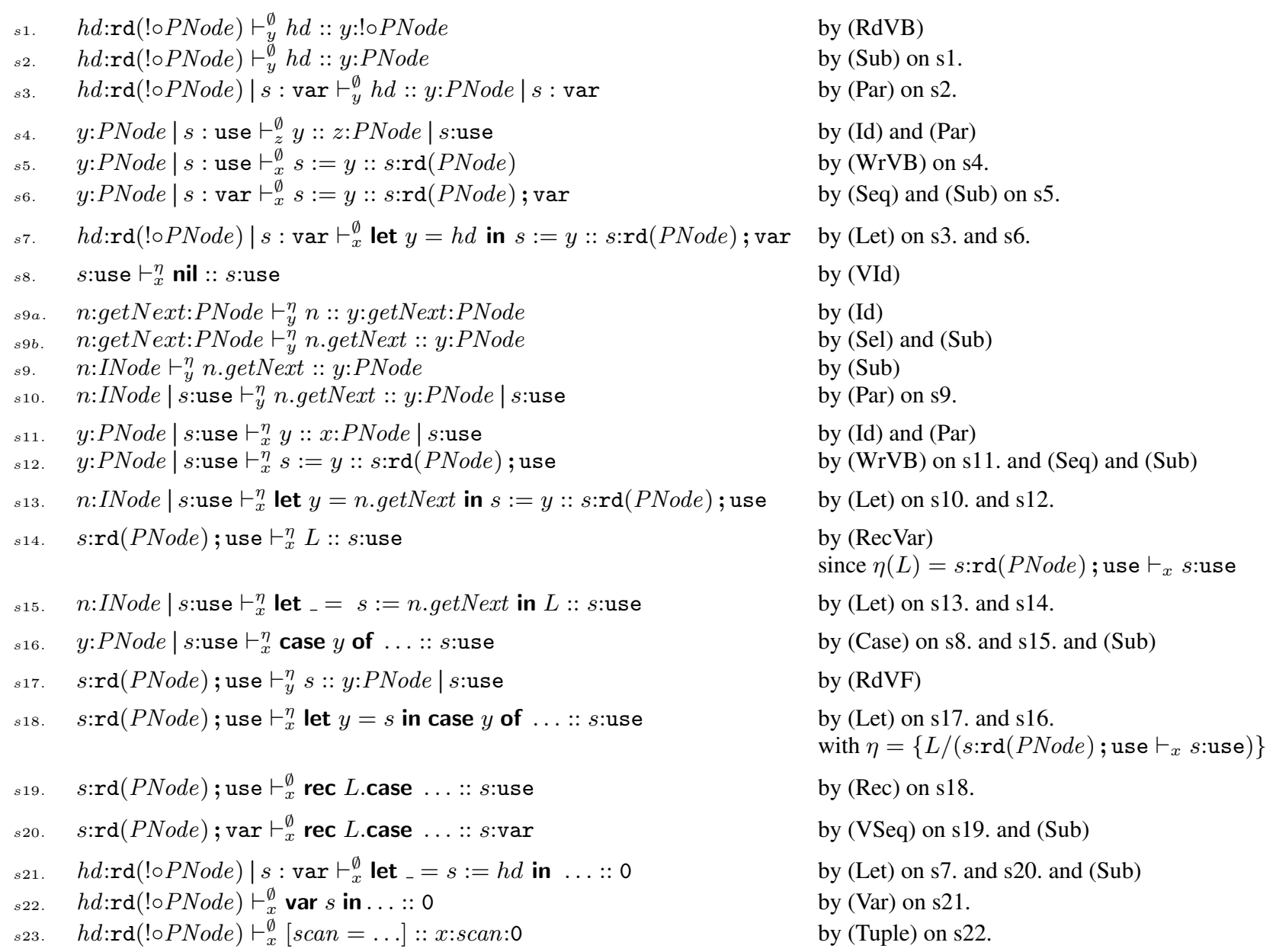

Figure 12. Typing derivation for "method" scan

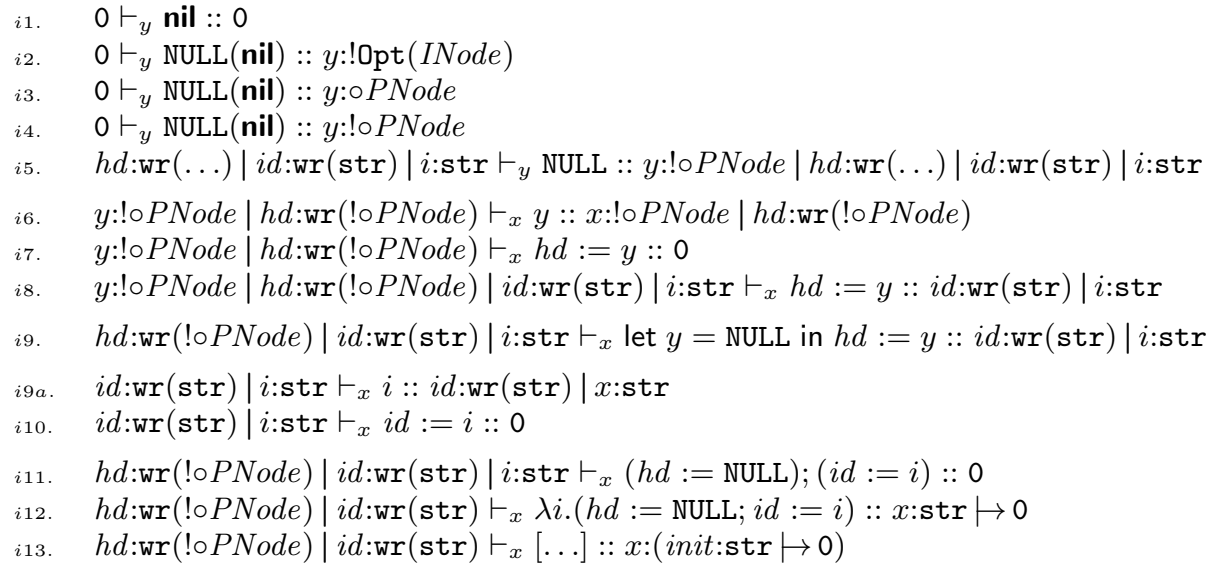

by (VStop) by (Option) on i1. and (VShr) by (Iso) on i2. by (VShr) on i3. by (Par) on i4. by (Id) and (Par) by (WrVF) on i6. by (Par) on i7.

by (Let) on i5. and i8. by (Id) and (Par) by (WrVF) on i9a. by (Let) on i9. and i10. by (Abs) on i11. by (Tuple) on i12.

Figure 13. Typing derivation for "method" init 

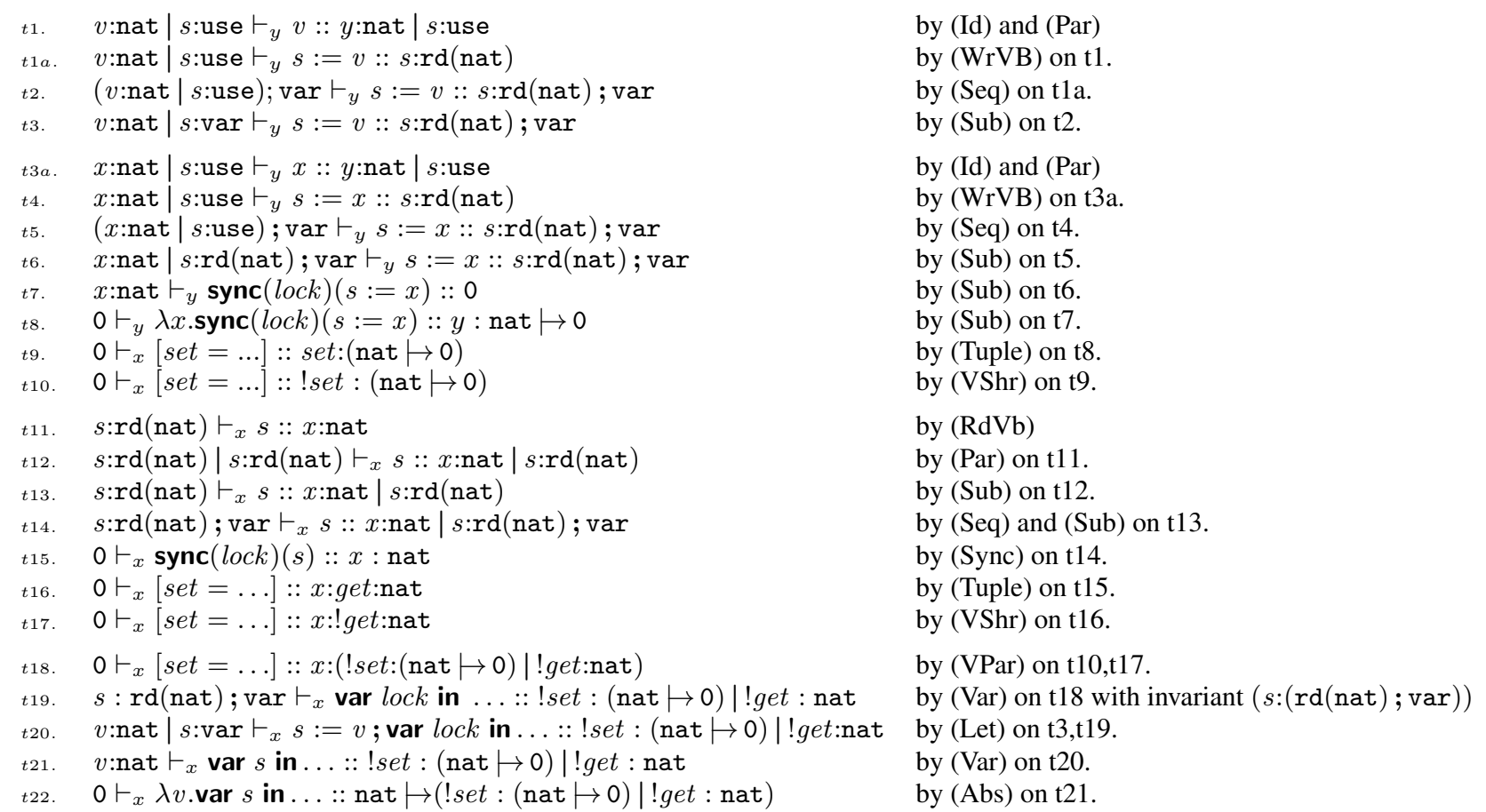

Figure 14. Typing derivation for function atomic 